\title{
Hubble flow variance and the cosmic rest frame
}

\author{
David L. Wiltshire, ${ }^{1}$ Peter R. Smale, ${ }^{1}$ Teppo Mattsson, ${ }^{1}$ and Richard Watkins ${ }^{2}$ \\ ${ }^{1}$ Department of Physics $\&$ Astronomy, University of Canterbury, \\ Private Bag 4800, Christchurch 8140, New Zealand \\ ${ }^{2}$ Department of Physics, Willamette University, Salem, OR 97301, USA
}

\begin{abstract}
We characterize the radial and angular variance of the Hubble flow in the COMPOSITE sample of 4534 galaxies, on scales in which much of the flow is in the nonlinear regime. With no cosmological assumptions other than the existence of a suitably averaged linear Hubble law, we find with decisive Bayesian evidence $(\ln B \gg 5$ ) that the Hubble constant, when averaged in independent spherical shells, is closer to its asymptotic value when referred to the rest frame of the Local Group (LG), rather than the standard rest frame of the Cosmic Microwave Background (CMB). An exception occurs for radial shells in the range $40 h^{-1}-60 h^{-1} \mathrm{Mpc}$. Angular averages reveal a dipole structure in the Hubble flow, whose amplitude changes markedly over the range $32 h^{-1}-62 h^{-1} \mathrm{Mpc}$. Whereas the LG frame dipole is initially constant and then decreases significantly, the CMB frame dipole initially decreases but then increases. The map of angular Hubble flow variation in the LG rest frame is found to coincide with that of the residual CMB temperature dipole, with correlation coefficient -0.92 . These results are difficult to reconcile with the standard kinematic interpretation of the motion of the Local Group in response to the clustering dipole, but are consistent with a foreground nonkinematic anisotropy in the distance-redshift relation of $0.5 \%$ on scales up to $65 \mathrm{~h}^{-1} \mathrm{Mpc}$. Effectively, the differential expansion of space produced by nearby nonlinear structures of local voids and denser walls and filaments cannot be reduced to a local boost. This hypothesis suggests a reinterpretation of bulk flows, which may potentially impact on calibration of supernova distances, anomalies associated with large angles in the CMB anisotropy spectrum, and the dark flow inferred from the kinematic Sunyaev-Zel'dovich effect. It is consistent with recent studies that find evidence for a nonkinematic dipole in the distribution of distant radio sources.
\end{abstract}

PACS numbers: 98.80.-k 98.80.Es 04.20.-q

\section{INTRODUCTION}

It is usually assumed that the cosmic microwave background (CMB) dipole [1, 2] is generated entirely by our own local peculiar velocity. A local boost by the opposite velocity then defines the cosmic rest frame in which we can be considered to be comoving observers in the background geometry of homogeneous isotropic Friedmann-Lemaître-Robertson-Walker (FLRW) model. Indeed measurements of cosmological redshifts are routinely transformed to the CMB frame. According to the assumptions implicit in such a transformation, the cosmic rest frame so defined should also be the frame in which the Hubble flow is most uniform, with minimal statistical variations as compared to other choices of the standard of rest.

Our understanding of the Hubble flow is, however, greatly complicated by the fact that the universe is not completely homogeneous. Rather it appears to only be homogeneous in some statistical sense, when one averages on scales $\gtrsim 100 h^{-1} \mathrm{Mpc}$, the transition scale still being a matter of debate [3 $[6]$. At scales below or comparable to the scale of statistical homogeneity a complex pattern of variance in the Hubble flow is observed. In the standard manner of thinking about the problem, Hubble flow variance is interpreted as a field of peculiar velocities of galaxies with respect to the expansion law of a FLRW model, which is linear on scales up to $z \sim 0.1$, well above the scale of statistical homogeneity. The CMB rest frame
Physical Review D 88, 083529 (2013)

sets the standard of rest for a comoving observer at our location in defining the leading order linear Hubble law. A great deal of observational effort has gone into understanding the nearby peculiar motions so derived; see, e.g., [7-17.

Some studies of peculiar velocities [18 21] have found results which indicate persistent bulk flows extending to very large scales, and which are potentially in conflict with the expectations of the perturbed FLRW model that underlies the standard Lambda Cold Dark Matter $(\Lambda \mathrm{CDM})$ cosmology. Using the large COMPOSITE data set of mainly non-SneIa galaxy distances Watkins, Feldman and Hudson [18] report a large bulk flow of $407 \pm 81 \mathrm{~km} \mathrm{~s}^{-1}$ toward $\ell=287^{\circ} \pm 9^{\circ}, b=8^{\circ} \pm 6^{\circ}$, with $90 \%$ of the sample within a $107 h^{-1} \mathrm{Mpc}$ sphere.

Different data sets and methods of analysis produce different, sometimes contradictory, results. For example, Davis and Nusser [22] and Ma and Scott [23] analyse samples which include large subsamples of the COMPOSITE sample, such as $\mathrm{SF}++[9]$. Applying different methods they find bulk flows consistent in direction with Watkins, Feldman and Hudson [18, 19] but with different amplitudes, which are consistent with $\Lambda \mathrm{CDM}$ predictions. Davis and Nusser [22] find a bulk flow $333 \pm 38 \mathrm{~km} \mathrm{~s}^{-1}$ towards $(\ell, b)=\left(276^{\circ}, 14^{\circ}\right) \pm 3^{\circ}$ within a $40 h^{-1} \mathrm{Mpc}$ sphere, and $257 \pm 44 \mathrm{~km} \mathrm{~s}^{-1}$ towards $(\ell, b)=\left(279^{\circ}, 10^{\circ}\right) \pm 3^{\circ}$ within a $100 h^{-1} \mathrm{Mpc}$ sphere; Ma and Scott [23] find amplitudes in the range $220-370 \mathrm{~km} \mathrm{~s}^{-1}$ in four different samples in an average direction $(\ell, b)=\left(280^{\circ} \pm 8^{\circ}, 5^{\circ} \pm 6^{\circ}\right)$ within a $80 h^{-1} \mathrm{Mpc}$ sphere. Turnbull et al. 24] have 
made a study using 245 type Ia supernova (SneIa) distances on somewhat larger scales $r \lesssim 190 h^{-1} \mathrm{Mpc}$. They find a bulk flow $249 \pm 76 \mathrm{~km} \mathrm{~s}^{-1}$ in the direction $\ell=$ $319^{\circ} \pm 18^{\circ}, b=7^{\circ} \pm 14^{\circ}$, which is consistent with the predictions of the $\Lambda \mathrm{CDM}$ model, but also marginally consistent with the larger bulk flow of Watkins, Feldman and Hudson [18, 19]. The results of Turnbull et al. [24] appear to be inconsistent, however, with the larger amplitude bulk flow of $600-1,000 \mathrm{~km} \mathrm{~s}^{-1}$ found by Kashlinsky et al. 20, 21] using the kinematic Sunyaev-Zel'dovich effect, principally on larger scales $120 \lesssim r \lesssim 600 h^{-1} \mathrm{Mpc}$.

In the above papers and elsewhere in the literature, with very few exceptions [25, 26], variance in the Hubble flow is generally attributed to peculiar velocities whose radial components are defined as deviations from a linear Hubble law

$$
v_{\mathrm{pec}}=c z-H_{0} r
$$

where $z$ is the redshift, $c$ the speed of light and $r$ an appropriate distance measure. Such a definition implicitly makes a strong assumption about spacetime geometry, namely on the scales of interest spatial curvature can be neglected and the redshift associated with the Hubble expansion can be treated in the manner of a recession velocity as in special relativity.

From the point of view of general relativity, without any a priori assumptions about the background geometry, such an assumption must be questioned. In general relativity in an arbitrary spacetime background the only velocities that are uniquely related to observables are those corresponding to local boosts at a point. Given that the dust approximation is not rigorously defined for the complex cosmic web of voids, walls and filaments that constitute the present day universe on $\lesssim 100 h^{-1} \mathrm{Mpc}$ scales [27], then the extension of the concept of a velocity in (11) over the vast distances over which space is expanding is merely an ansatz, whose validity remains to be justified. In any general relativistic framework there must be some local peculiar velocities, which arise from the local dynamics of galaxies within bound clusters. However, there is no a priori reason for assuming that all redshifts on scales $\lesssim 100 h^{-1} \mathrm{Mpc}$ can be treated in terms of a simple Doppler shift in Euclidean space, which is in practice the method of analysis adopted by most observationalists.

From the point of view of general relativity, variance in the Hubble flow in its nonlinear regime is more naturally viewed as the differential expansion of regions of different local densities, which have experienced different local expansion histories over the billions of years that have elapsed since the density field was close to uniform. While particular geometrical assumptions would lead to the standard FLRW geometry with Newtonian perturbations, the lack of convergence of bulk flows to the CMB dipole and the puzzle of several possible anomalies associated both with bulk flows and the large angle multipoles of the CMB anisotropy spectrum [20, 28 33], suggest that one should reconsider the problem from first principles.
In this paper we will therefore reanalyse the largest available data set, the COMPOSITE sample of Watkins, Feldman and Hudson [18, 19], from a fresh perspective. While the particular analysis we adopt is one which is naturally suggested by the cosmological model of Refs. [34 37], our actual analysis is independent of any cosmological model assumptions other than the most elementary one that a suitably defined average linear Hubble law exists.

The plan of the paper is as follows. In Sec. II we first consider the spherical (monopole) variation of the Hubble law in independent radial shells, obtain statistical bounds on the differences between the CMB and Local Group (LG) frames, and provide an explanation of the systematic differences between the two frames. In Sec. [II we consider angular averages, using a Gaussian window function average to estimate the ratios of large angle multipoles, and by fitting a dipole Hubble law in independent radial shells for those shells with a dominant dipole. We determine the statistical significance of the dipole and its amplitude in relation to the monopole variations. We also identify those structures in that might be responsible for the density gradient which induces both the monopole and dipole variations. In Sec. IV we check that the Hubble variance dipole correlates with the component of the CMB dipole usually attributed to the motion of the LG, with very strong significance. In Sec. V. given that our combined results do not support a purely kinematic response of the LG to the clustering dipole, we suggest a new alternative mechanism for the generation of this contribution to the CMB dipole. Finally in Sec.VI we discuss some potential implications of our results.

\section{SPHERICAL AVERAGES IN RADIAL SHELLS}

We adopt the point of view that on scales of order $10 h^{-1}-30 h^{-1} \mathrm{Mpc}$ the regional expansion history, and the regional average Hubble law should be determined primarily by the relevant regional average density. From the point of view of any observer, underdense voids will appear to be expanding faster than denser wall regions, on account of the wall regions having decelerated more. This is true independently of whether or not there is a homogeneous dark energy which acts to accelerate the expansion by the same amount in all regions.

The largest typical voids are shown by surveys to have a diameter $\sim 30 h^{-1} \mathrm{Mpc}$ [38 40]. Our galaxy is located in a filamentary sheet on the edge of a Local Void of at least this diameter, formed from a complex of three smaller voids 10 ].

Although the expansion rate just to the other side of a local void (wall) will appear faster (slower) than average, whole sky spherical averages that include many structures in different directions can be expected to have a reduced variance as compared to measurements in particular directions. Furthermore, once one also averages 
on radial scales a few times larger than the largest typical structures then the variance of the Hubble parameter averaged in spherical shells will reduce to a level consistent with individual measurement uncertainties. (See Fig. 1) This provides an operational definition of the scale of statistical homogeneity independent of any detailed cosmological model assumptions.

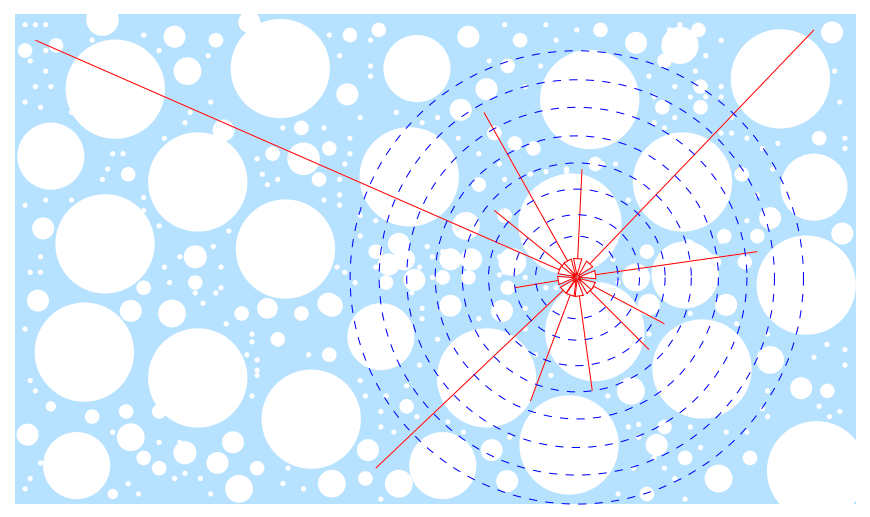

FIG. 1: Schematic diagram of spherical averaging. The universe is described as ensemble of filaments, walls and voids: expanding regions of different density which have decelerated by different amounts and therefore experience different local expansion rates at the present epoch. Observations show that the largest typical nonlinear structures are voids of $\sim 30 h^{-1} \mathrm{Mpc}$ 38 40], which occupy $40 \%$ of the volume of the present epoch universe. If one averages $c z / r$ in spherical shells (dotted lines) about a point then once the shells are a few times larger than the typical nonlinear structures, an average Hubble law with small statistical scatter is obtained (e.g., for longest null geodesics represented by arrowed lines), whereas there are considerable deviations for shells on scales comparable to the typical nonlinear structures. This approach is model independent since it makes no assumptions about the geometry of the universe on the scales in question.

Determining the scale of statistical homogeneity observationally is an interesting challenge and a matter of debate [3, 4], one of the issues being an appropriate definition of statistical homogeneity since there will always be some cosmic variance on the largest of scales. Any reasonable definition should encompass a notion of a transition between a nonlinear regime and a linear regime of inhomogeneous perturbations relative to an average almost homogeneous evolution, whether this is described by a FLRW model or not [27]. Since the largest typical nonlinear structures are voids of $30 h^{-1} \mathrm{Mpc}$ diameter, which occupy of order $40 \%$ of the volume of the present universe [38, 39], the scale of statistical homogeneity should be at least a few times larger. On the other hand, since the Baryon Acoustic Oscillation (BAO) feature is observed in galaxy clustering statistics in accord with the expectations of linear perturbation theory on a FLRW background, the scale of statistical homogeneity should necessarily be of the same order or smaller, i.e., $\lesssim 110 h^{-1} \mathrm{Mpc}$. This accords with a recent measurement of the scale of statistical homogeneity by Scrimgeour et al. [5] in the WiggleZ survey.

A study of the spherically averaged Hubble flow, as a function of radial distance was undertaken by $\mathrm{Li}$ and Schwarz [26] (henceforth LS08), using a subset of 54 distances from the Hubble Space Telescope (HST) Key project data 41]. Fig. 2 of LS08 shows the radial variation $\delta H(r)=\left(H(r)-H_{0}\right) / H_{0}$ that results from such an analysis, with data restricted to the range $r_{\min }<$ $r<r_{\max }$, where $r_{\min }=22.5 h^{-1} \mathrm{Mpc}$ and $r_{\max }=$ $130 h^{-1} \mathrm{Mpc}$. Furthermore, their fit is computed for redshifts referred to a single linear Hubble law $c z=H(r) r$ within a sphere of radius $r$, as $r$ is varied, and with redshifts referred to the CMB frame.

Our first aim here is to perform a similar analysis to LS08 using the COMPOSITE sample of cluster, group and galaxy distances compiled by Watkins, Feldman and Hudson [18] (henceforth WFH09) and slightly updated by Feldman, Watkins and Hudson [19] (henceforth FWH10). For each sample object, redshift, galactic latitude and longitude, distance, and distance uncertainty are given. Distance uncertainties are about $15 \%$ for most individual galaxies. We include all 4,534 data points outside the local group $r>2 h^{-1} \mathrm{Mpc}$, which extends the range of distances considered in LS08 to both smaller and larger values.

The data in the COMPOSITE sample combines nine independent, full-sky samples, nearly all major peculiar velocity surveys published to date. Although each survey uses a different distance measurement methodology, all of the surveys were shown to be statistically consistent with each other [18]. The survey of Ref. [42] was not included, as it gave inconsistent results [18. WFH09 provide a detailed discussion of the issues involved in combining subsamples with different characteristic depths and sky coverages. For our analysis it is important to note that outside the Zone of Avoidance (ZoA) the COMPOSITE sample has good all sky coverage, as is seen from Fig. 1 of FWH10, and is further discussed in Sec. IIA and Appendix $\mathrm{D}$ below.

\section{A. Methodology}

Our analysis will feature two key differences to that of LS08. Firstly, rather than simply performing the analysis in the CMB frame, we also perform the analysis in the local group (LG) and local sheet (LS) frames. A comparison of these frames is motivated by the Cosmological Equivalence Principle 36]: the LG frame corresponds to our "finite infinity region" 34], and should be close to the frame in which the variance in the Hubble flow is minimized in the approach to cosmological averages advocated in Refs. [34 36]. The LG has a small peculiar velocity of $66 \pm 24 \mathrm{~km} \mathrm{~s}^{-1}$ relative to the LS [10] within which a local Hubble flow is first defined.

Secondly, on account of the small number of data 
points, Li and Schwarz [26] included all data within a radius $r$, as $r$ was varied in steps between $r_{\text {min }}$ and $r_{\max }$. This has the effect that each binned data point shown in their Fig. 2 is correlated with the previous data point. With 4,534 available data points in the COMPOSITE sample such correlations can be avoided by the following technique: we will minimize the sum $\chi_{s}^{2}=\sum_{i}\left[\sigma_{i}^{-1}\left(r_{i}-c z_{i} / H\right)\right]^{2}$ with respect to $H$, as a means of fitting a Hubble law by a standard linear regression [43], in successive independent radial shells $r_{s}<r \leq r_{s+1}$. We consider the linear Hubble law with $r$ as a function of $z$ since all uncertainties have been included as distance uncertainties ${ }^{1}$ in the COMPOSITE sample. The value of the Hubble constant $H_{s}$ computed for the $s$ th shell is then

$$
H_{s}=\left(\sum_{i=1}^{N_{s}} \frac{\left(c z_{i}\right)^{2}}{\sigma_{i}^{2}}\right)\left(\sum_{i=1}^{N_{s}} \frac{c z_{i} r_{i}}{\sigma_{i}^{2}}\right)^{-1},
$$

where $\sigma_{i}$ denote individual distance uncertainties in $h^{-1} \mathrm{Mpc}$.

The total uncertainty for $H_{s}$ in each shell ${ }^{2}, \bar{\sigma}_{s}$, is determined by adding the following uncertainties in quadrature: (i) the uncertainty determined by standard error propagation for the linear fit (2) in the sth shell

$$
\bar{\sigma}_{1 s}=\left(\sum_{i=1}^{N_{s}} \frac{\left(c z_{i}\right)^{2}}{\sigma_{i}^{2}}\right)^{3 / 2}\left(\sum_{i=1}^{N_{s}} \frac{c z_{i} r_{i}}{\sigma_{i}^{2}}\right)^{-2},
$$

and (ii) a zero point uncertainty

$$
\bar{\sigma}_{0 s}=H_{s} \frac{\sigma_{0}}{\bar{r}_{s}}
$$

where $\bar{r}_{s}=\left(\sum_{i=1}^{N_{s}} \frac{r_{i}}{\sigma_{i}^{2}}\right)\left(\sum_{i=1}^{N_{s}} \frac{1}{\sigma_{i}^{2}}\right)^{-1}$ is the weighted mean distance of the $N_{s}$ points in the sth shell and $\sigma_{0}=0.201 h^{-1} \mathrm{Mpc}$ is the distance uncertainty arising

\footnotetext{
${ }^{1}$ While the measurement uncertainties in redshifts are negligible, using the standard peculiar velocity framework a uniform velocity noise uncertainty was added in quadrature to $H_{0} \sigma_{i}$ in FWH10 in defining the maximum likelihood weights. In the peculiar velocity framework galaxy motions are modeled using linear theory. The velocity noise term then accounts for the fact that individual peculiar velocities deviate from the local value of the linear peculiar velocity field due to small-scale nonlinear motions. Here we are not using linear theory to model deviations from a single global linear Hubble law, so the addition of velocity noise to our analysis is unnecessary. In our framework we would still have to take into account the noise due to peculiar velocities of galaxies within gravitationally bound clusters. However, in the COMPOSITE data set this has already been accounted for in gravitationally bound systems by assigning distances and associated uncertainties to clusters, rather than to the individual galaxies within the clusters.

${ }^{2}$ We use an overbar for uncertainties in the Hubble constant obtained by either radial or angular averages, to distinguish them from the distance uncertainties in individual data points.
}

from the $20 \mathrm{~km} \mathrm{~s}^{-1}$ uncertainty in the heliocentric peculiar velocity of both the Local Group and Local Sheet as given in Ref. [10] added in quadrature to the $0.4 \%$ uncertainty in the magnitude of the CMB dipole $^{3}[2]$.

The uncertainty (4) is included since the Hubble law is necessarily determined by a linear fit through the origin for each shell. The local velocity uncertainty when divided by $H_{0}$ provides an additional distance uncertainty in the mean distance of each shell relative to the origin, and the related uncertainty (44) in the mean slope $H_{s}$. This uncertainty is significant for shells close to the origin, but much smaller for shells at large radii for which the mean distance has a long lever arm.

In Eqs. (3), (4) $H_{0}=100 \mathrm{hm} \mathrm{s}^{-1} \mathrm{Mpc}^{-1}$ represents the normalization used to convert velocity uncertainties to distance uncertainties in the COMPOSITE data set. One other important issue is the asymptotic value of the Hubble constant in each frame to which the variance in the Hubble flow should be normalized, as this global value should have its own uncertainty. To this end we have divided the data in $12.5 h^{-1} \mathrm{Mpc}$ wide shells out to those radial distances of order $150 h^{-1} \mathrm{Mpc}$, for two different choices of shells differing by the initial inner shell boundary, as shown in Table [] The penultimate shell, 10 or $10^{\prime}$, has been made wider so that it contains a similar number of points to most inner shells.

For both choices of shells 91 data points with $r>$ $156.25 h^{-1} \mathrm{Mpc}$ in shell 11 have been used to determine the mean asymptotic value of the Hubble constant, $\bar{H}_{0}$, and its uncertainty. The inner boundary of this shell must be chosen at a sufficiently large distance that it is greater than the scale of statistical homogeneity. Thus we take the inner shell radius to be larger than the baryon acoustic oscillation (BAO) scale, that being the largest scale which could reasonably modify the gross features of the local Hubble flow ${ }^{4}$. Furthermore, in the CMB frame the asymptotic Hubble constant should match the

${ }^{3}$ Very slightly different temperature dipoles are given by Fixsen et al. 2] and Bennett et al. [44]. Since much of the COMPOSITE data set was determined before the Bennett et al. [44] result, we assume that it has been normalized relative to the heliocentric frame using the Fixsen et al. [2] value of $v_{\mathrm{CMB}}=371 \mathrm{~km} \mathrm{~s}^{-1}$ in a direction $\ell=264.14^{\circ}, b=48.26^{\circ}$, which is the standard used in the NASA/IPAC Extragalactic Database. Our heliocentric velocities of the LG and LS are taken from Ref. [10] as $v_{\mathrm{LG}}=318.6 \mathrm{~km} \mathrm{~s}^{-1}$ towards $\ell=106^{\circ}, b=-6^{\circ}$, and $v_{\mathrm{LS}}=318.2 \mathrm{~km} \mathrm{~s}^{-1}$ towards $\ell=95^{\circ}, b=-1^{\circ}$ respectively.

${ }^{4}$ In seeking convergence of bulk flows to the CMB dipole, researchers working in the peculiar velocity framework are currently considering the influence of the Shapley Concentration on our local motion. Since Shapley is at a distance of $138 h^{-1} \mathrm{Mpc}$, such a large scale correlation would have to represent a very unusual fluctuation relative to the statistical homogeneity scale if the standard framework were correct. The standard framework has focused attention on the largest overdense structures. However, the very underdense regions are equally important in determining averages and are naturally incorporated in the spherical averaging approach (c.f. Fig. 1). 


\begin{tabular}{|c|c|c|c|c|c|c|c|c|c|c|c|}
\hline Shell $s$ & 1 & 2 & 3 & 4 & 5 & 6 & 7 & 8 & 9 & 10 & 11 \\
\hline$N_{s}$ & 92 & 505 & 514 & 731 & 819 & 562 & 414 & 304 & 222 & 280 & 91 \\
\hline$r_{s}\left(h^{-1} \mathrm{Mpc}\right)$ & 2.00 & 12.50 & 25.00 & 37.50 & 50.00 & 62.50 & 75.00 & 87.50 & 100.00 & 112.50 & 156.25 \\
\hline $\bar{r}_{s}\left(h^{-1} \mathrm{Mpc}\right)$ & 5.43 & 16.33 & 30.18 & 44.48 & 55.12 & 69.24 & 81.06 & 93.75 & 105.04 & 126.27 & 182.59 \\
\hline$\left(H_{s}\right)_{\mathrm{CMB}}$ & 173.9 & 111.1 & 110.4 & 104.1 & 102.7 & 103.8 & 102.1 & 102.8 & 104.4 & 102.1 & 100.1 \\
\hline$\left(\bar{\sigma}_{s}\right)_{\mathrm{CMB}}$ & 6.8 & 1.5 & 1.1 & 0.7 & 0.7 & 0.8 & 0.9 & 0.9 & 1.0 & 0.8 & 1.7 \\
\hline$\left(Q_{s}\right)_{\mathrm{CMB}}$ & 0.000 & 0.000 & 0.000 & 0.037 & 0.985 & 0.997 & 1.000 & 1.000 & 1.000 & 0.979 & 0.999 \\
\hline$\left(\chi^{2} / \nu\right)_{\mathrm{CMB}}$ & 24.639 & 5.802 & 1.741 & 1.096 & 0.896 & 0.841 & 0.593 & 0.604 & 0.630 & 0.835 & 0.581 \\
\hline$\left(H_{s}\right)_{\mathrm{LG}}$ & 117.9 & 103.1 & 106.5 & 105.5 & 104.8 & 102.1 & 102.8 & 103.2 & 103.7 & 102.4 & 101.0 \\
\hline$\left(\bar{\sigma}_{s}\right)_{\mathrm{LG}}$ & 4.6 & 1.4 & 1.0 & 0.7 & 0.7 & 0.7 & 0.9 & 0.9 & 1.0 & 0.8 & 1.7 \\
\hline$\left(Q_{s}\right)_{\mathrm{LG}}$ & 0.000 & 0.000 & 0.000 & 0.000 & 0.998 & 0.940 & 1.000 & 1.000 & 1.000 & 0.993 & 0.999 \\
\hline$\left(\chi^{2} / \nu\right)_{\mathrm{LG}}$ & 23.656 & 7.767 & 2.185 & 1.419 & 0.864 & 0.909 & 0.594 & 0.542 & 0.622 & 0.803 & 0.590 \\
\hline $\ln B\left(r \geq r_{s}\right)$ & 58.62 & 16.95 & 8.43 & 1.71 & 1.98 & 2.71 & 1.64 & 1.78 & 1.67 & 0.49 & \\
\hline Shell $s$ & $1^{\prime}$ & $2^{\prime}$ & $3^{\prime}$ & $4^{\prime}$ & $5^{\prime}$ & $6^{\prime}$ & 7 & $8^{\prime}$ & $9^{\prime}$ & $10^{\prime}$ & 11 \\
\hline$N_{s}$ & 321 & 513 & 553 & 893 & 681 & 485 & 343 & 273 & 164 & 206 & 91 \\
\hline$r_{s}\left(h^{-1} \mathrm{Mpc}\right)$ & 6.25 & 18.75 & 31.25 & 43.75 & 56.25 & 68.75 & 81.25 & 93.75 & 106.25 & 118.75 & 156.25 \\
\hline $\bar{r}_{s}\left(h^{-1} \mathrm{Mpc}\right)$ & 12.26 & 23.46 & 37.61 & 49.11 & 61.74 & 73.92 & 87.15 & 99.12 & 111.95 & 131.49 & 182.59 \\
\hline$\left(H_{s}\right)_{\mathrm{CMB}}$ & 120.8 & 108.8 & 105.9 & 103.6 & 104.2 & 102.6 & 102.7 & 103.8 & 102.0 & 102.3 & 100.1 \\
\hline$\left(\bar{\sigma}_{s}\right)_{\mathrm{CMB}}$ & 2.1 & 1.2 & 0.9 & 0.7 & 0.8 & 0.8 & 0.9 & 0.9 & 1.0 & 0.9 & 1.7 \\
\hline$\left(Q_{s}\right)_{\mathrm{CMB}}$ & 0.000 & 0.000 & 0.000 & 0.555 & 0.959 & 1.000 & 1.000 & 1.000 & 0.992 & 0.997 & 0.999 \\
\hline$\left(\chi^{2} / \nu\right)_{\mathrm{CMB}}$ & 9.496 & 2.506 & 1.421 & 0.993 & 0.908 & 0.633 & 0.624 & 0.658 & 0.754 & 0.745 & 0.581 \\
\hline$\left(H_{s}\right)_{\mathrm{LG}}$ & 103.5 & 103.5 & 103.9 & 106.6 & 103.9 & 102.0 & 103.2 & 103.6 & 101.6 & 102.7 & 101.0 \\
\hline$\left(\bar{\sigma}_{s}\right)_{\mathrm{LG}}$ & 1.8 & 1.1 & 0.9 & 0.7 & 0.8 & 0.8 & 0.9 & 0.9 & 1.0 & 0.9 & 1.7 \\
\hline$\left(Q_{s}\right)_{\mathrm{LG}}$ & 0.000 & 0.000 & 0.000 & 0.031 & 0.960 & 1.000 & 1.000 & 1.000 & 0.996 & 0.999 & 0.999 \\
\hline$\left(\chi^{2} / \nu\right)_{\mathrm{LG}}$ & 11.427 & 3.246 & 1.792 & 1.090 & 0.907 & 0.701 & 0.592 & 0.608 & 0.728 & 0.711 & 0.590 \\
\hline $\ln B\left(r \geq r_{s}\right)$ & 30.09 & 8.99 & 3.22 & 0.92 & 2.47 & 1.68 & 1.37 & 1.30 & 0.64 & 0.39 & \\
\hline
\end{tabular}

TABLE I: Hubble flow variation in radial shells in CMB and LG frames. Spherical averages (2) are computed for two different choices of shells, $r_{s}<r \leq r_{s+1}$, the second choice being labeled by primes. In each case we tabulate the inner shell radius, $r_{s}$; the weighted mean distance, $\bar{r}_{s}$; the shell Hubble constants, $\left(H_{s}\right)_{\mathrm{CMB}}$ and $\left(H_{s}\right)_{\mathrm{LG}}$ in the CMB and LG frames, and their uncertainties determined by linear regression within each shell, together with its "goodness of fit" probability $Q_{s}$ and reduced $\chi^{2}$ (for $\left.\nu=N_{s}-1\right) ; \ln B$ where $B$ is the Bayes factor for the relative probability that the LG frame has more uniform $\delta H_{s}=0$ than the CMB frame when $\chi^{2}$ is summed in all shells with $r>r_{s} . H_{s}$ and $\bar{\sigma}_{s}$ are given in units $h \mathrm{kms}^{-1} \mathrm{Mpc}^{-1}$.

$100 h^{-1} \mathrm{Mpc}$ normalization used in the COMPOSITE data set. This is indeed satisfied by our choice. We find $\bar{H}_{0}=(100.1 \pm 1.7) h \mathrm{~km} \mathrm{~s}^{-1} \mathrm{Mpc}^{-1}$ for the CMB frame and $\bar{H}_{0}=(101.0 \pm 1.7) h \mathrm{~km} \mathrm{~s}^{-1} \mathrm{Mpc}^{-1}$ for the LG/LS frames. We thus see that although the LG/LS value is $1 \%$ larger than the CMB value, both values agree within uncertainties, and also with the distance normalization assumed in compiling the COMPOSITE sample.

We do not determine $\bar{H}_{0}$ from the whole COMPOSITE sample, since it is dominated by points in the foreground, with a mean weighted distance of $15.05 h^{-1} \mathrm{Mpc}$. The fit of a single linear Hubble law to the whole sample of 4,534 points gives $\bar{H}_{0}=(108.9 \pm 1.5) h \mathrm{~km} \mathrm{~s}^{-1} \mathrm{Mpc}^{-1}$ in the CMB frame or $\bar{H}_{0}=(104.4 \pm 1.4) h \mathrm{~km} \mathrm{~s}^{-1} \mathrm{Mpc}^{-1}$ in the $\mathrm{LG}$ and LS frames. It is precisely because voids dominate the volume of space that we expect radial averages on scales comparable to the diameters of the largest typical voids to skew the simple linear average to values greater than the asymptotic global value. This is confirmed by the full sample simple linear fit. Our purpose is to more carefully quantify the foreground Hubble flow variance.

The key statistical point about the determination of the mean asymptotic value, $\bar{H}_{0}$, in each case is that its uncertainty provides a significant contribution to the total uncertainty in the relative variation of the Hubble parameter in the $s$ th shell

$$
\delta H_{s}=\left(H_{s}-\bar{H}_{0}\right) / \bar{H}_{0} .
$$

We have checked that the angular sky coverage of the sample is consistent in individual shells. This is important since we could get spurious results if the data in any shell was limited to one side of the sky, and potentially dominated by particular structures. In Fig. 2 we plot figures similar to Fig. 1 of FWH10, which shows the sky coverage within each of the unprimed shells of Sec. [II We use a Mollweide projection in galactic coordinates $(\ell, b)$ with $\ell=360^{\circ}$ on the extreme left and $\ell=0^{\circ}$ on the extreme right. Additional peculiar velocity information is encoded in the relative sizes and colours of the data points.

We see that angular sky coverage is consistent in almost all shells, with some large gaps only the innermost shell $1, r<12.5 h^{-1} \mathrm{Mpc}$. While shells 9 and 10 contain 222 and 280 points respectively, i.e., of order half the number in most other shells, a Monte Carlo analysis discussed in Appendix D establishes that there is still sufficient data in these shells to support the existence of 


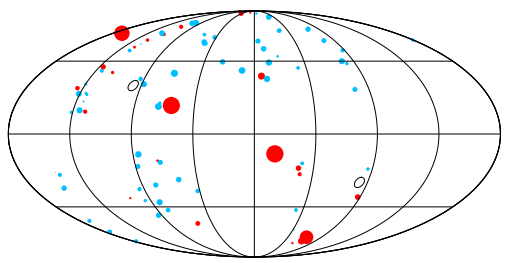

(a) 1: $6.25-18.75 h^{-1} \operatorname{Mpc} N=321$.

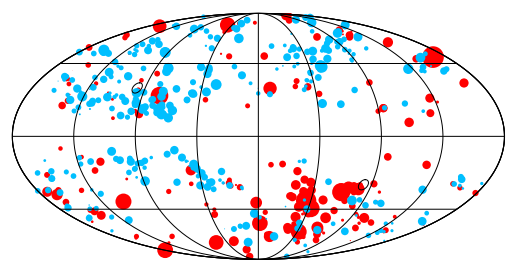

(c) 3: $31.25-43.75 h^{-1} \mathrm{Mpc} N=553$.

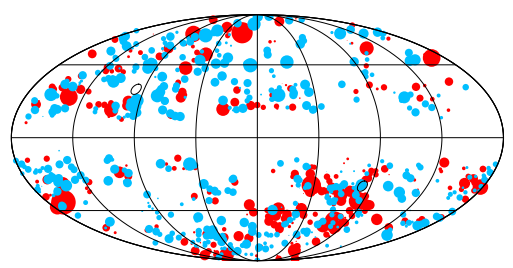

(e) 5: $56.25-68.75 h^{-1} \operatorname{Mpc} N=681$.

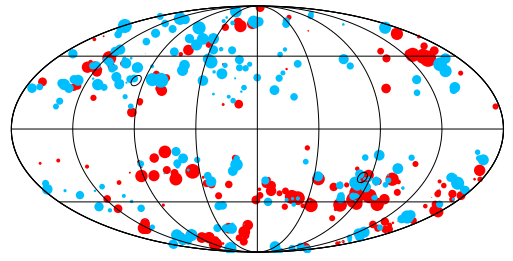

(g) 7: $81.25-93.75 h^{-1} \operatorname{Mpc} N=343$.

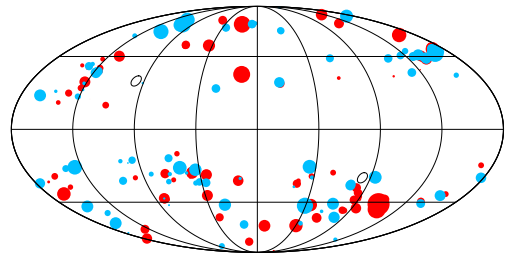

(i) 9: $106.25-118.25 h^{-1} \mathrm{Mpc} N=164$

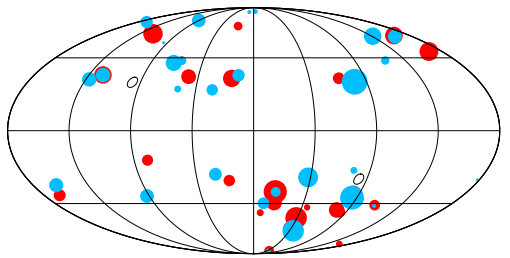

(k) 11: $156.25-417.4 h^{-1} \operatorname{Mpc} N=91$.

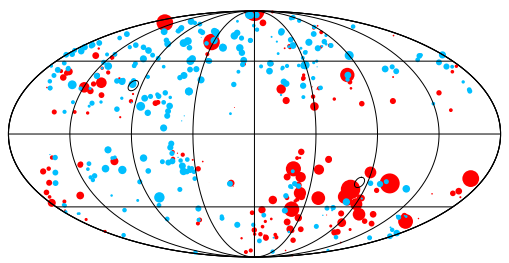

(b) 2: $18.75-31.25 h^{-1} \operatorname{Mpc} N=513$.

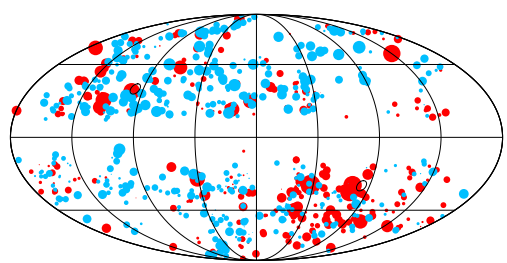

(d) 4: $43.75-56.25 h^{-1} \operatorname{Mpc} N=893$.

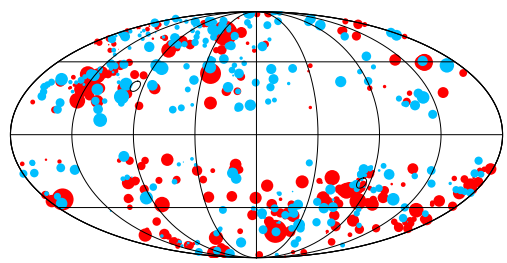

(f) 6: $68.75-81.25 h^{-1} \operatorname{Mpc} N=485$.

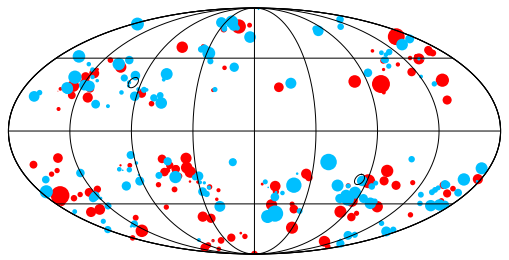

(h) 8: $93.75-106.25 h^{-1} \operatorname{Mpc} N=273$.

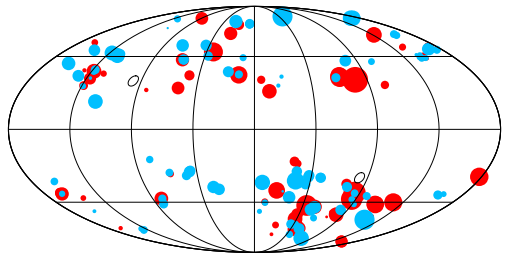

(j) 10: $118.25-156.25 h^{-1} \operatorname{Mpc} N=206$. 
a dipole feature in the CMB frame in at the $97.6 \%$ and $99.7 \%$ confidence levels respectively. There are insufficient data points in the outermost shell 11 to reliably distinguish any angular variations. However, this shell is only used as a check on the asymptotic spherically averaged Hubble constant, for which there are no statistical problems, the goodness of fit statistic being 0.999.

For the primed shells, where the inner boundary is offset by $6.25 h^{-1} \mathrm{Mpc}$ there is no sky coverage problem, even in the innermost shell. We will retain the (unprimed) shell 1 in our analysis, but our statistical conclusions will not rely on it.

\section{B. Results}

The results of our analysis are shown in Fig. 3, where we plot $\delta H_{s}$ for both the CMB and LG frames, for the two independent choices of shells given in Table प along with the difference $\left(H_{s}\right)_{\mathrm{CMB}}-\left(H_{s}\right)_{\mathrm{LG}}$. We computed the result for the LS also; it is essentially indistinguishable from the LG frame.

The values of $\delta H_{s}$ are positive, consistent with the results of LS08, and consistent with the fact that in a universe whose volume is dominated by voids a spherical average will inevitably include more voids than the denser filaments and walls if one averages on scales comparable to the diameters of the largest typical voids, leading to a higher than average $H_{s}$ as compared to the asymptotic value $\bar{H}_{0}$.

It is clear that the variance of the spherically averaged LG or LS frame Hubble flow is less than that of the CMB frame. In both frames the Hubble flow averaged in spherical shells gives $\delta H_{s}$ within $2 \sigma$ of uniform in almost all shells ${ }^{5}$ for $\bar{r}_{s} \geq 55.1 h^{-1} \mathrm{Mpc}$. However, particularly for values $\bar{r}_{s}<37.6 h^{-1} \mathrm{Mpc}$, the LG/LS frame has $\delta H_{s}$ much closer to uniform than the CMB frame, and the average LG/LS frame flow is even within $1.2 \sigma$ of uniform in the range $12.3 h^{-1}<\bar{r}_{s} \leq 23.5 h^{-1} \mathrm{Mpc}$, whereas the average CMB frame flow is $4.0 \sigma-7.0 \sigma$ from uniform in the same range. Since the Local Sheet itself is defined within $r<6.25 h^{-1} \mathrm{Mpc}$, this is not a result that would be readily expected with the standard interpretation of peculiar velocities.

The statistical significance of the relative uniformity of the averaged flow in the two frames can now be determined by summing the mean square differences from a

\footnotetext{
5 The one small exception is that $\delta H_{\mathrm{CMB}}$ is $2.1 \sigma$ from uniform for shell 9 with $100 h^{-1}<r \leq 112.5 h^{-1} \mathrm{Mpc}$ and $\bar{r}_{9}=$ $105.0 \mathrm{~h}^{-1} \mathrm{Mpc}$. In general, the LG frame flow is still somewhat closer to uniform than the CMB frame flow in the outer regions. For all shells with $\bar{r}_{s} \geq 69.2 h^{-1} \mathrm{Mpc}$ the LG frame flow is within $1.36 \sigma$ of uniform.
}

uniform $\delta H=0$ expectation,

$$
\chi^{2}\left(r_{s}\right)=\sum_{j=s}^{12} \frac{\bar{H}_{0}^{4} \delta H_{j}^{2}}{\bar{H}_{0}^{2} \bar{\sigma}_{H_{j}}^{2}+H_{j}^{2} \bar{\sigma}_{\bar{H}_{0}}^{2}},
$$

for each choice of rest frame in all shells outside an inner cutoff shell, $r_{s}$, as the inner cutoff is varied. An inner cutoff is commonly applied to eliminate the contribution of large peculiar velocities near the origin, and given the reinterpretation we follow in this paper, the effect of varying the cutoff is particularly interesting. The probability, $P_{\mathrm{CMB}}\left(r_{s}\right)$ or $P_{\mathrm{LG}}\left(r_{s}\right)$, of a uniform Hubble flow for each choice of rest frame and cutoff is then calculated directly from the complementary incomplete gamma function for the chi square distribution with the relevant number of degrees of freedom. A Bayes factor $B\left(r_{s}\right)=P_{\mathrm{LG}} / P_{\mathrm{CMB}}$ for each choice of inner cutoff is determined for the two independent choices of shells in Table I] The resulting values of $\ln B$ are tabulated in Table \ and plotted as a function of $r_{s}$ in Fig. 4. We also determined $P_{\mathrm{LS}} / P_{\mathrm{CMB}}$ for the LS relative to the CMB; however, the values obtained gave Bayes factors which were essentially indistinguishable from those tabulated for the LG relative to the CMB.

Fig. 4 reveals a number of interesting features. The fact that overall the LG frame is more uniform is demonstrated by $\ln B$ being everywhere positive. If we consider a large inner cutoff, $r_{s} \geq 106.25 h^{-1} \mathrm{Mpc}$ then the difference in uniformity of the two frames has $\ln B \leq 1$, which is not statistically significant. With cutoffs in the range $37.5 h^{-1} \leq r_{s} \leq 100 h^{-1} \mathrm{Mpc}$ we find $1<\ln B<3$ with positive evidence in favour of the LG frame being the more uniform. Bringing the cutoff down to $r_{s}=$ $37.5 h^{-1} \mathrm{Mpc}$ gives $\ln B=3.6$ increasing the Bayesian evidence to strong. For cutoffs $r_{s} \leq 25 h^{-1} \mathrm{Mpc}$ the Bayesian evidence becomes very strong, $\ln B>5$. Different adjectives are used to describe the strongest Bayesian evidence [45, 46]; since $\ln B>10$ for any inner cutoff with $r_{s}<14.5 h^{-1} \mathrm{Mpc}$ Jeffreys' terminology of "decisive evidence" in favour of the relative uniformity of the Hubble flow in the LG frame seems to be appropriate.

We must be careful, however, in the determination of statistical confidence, since there are also statistically significant departures from uniformity in the LG and LS frames also, as is consistent with the presence of foreground structures.

The nonlinear effects of the foreground structures can be seen by computing the goodness of fit probability, $Q_{s}$, given by the complementary incomplete gamma function for $\chi_{s}^{2}$ in shell $s$ with $\nu=N_{s}-1$ degrees of freedom. In Table \a bad linear fit is indicated in both the CMB and LG frames for shells $s \leq 4$ (unprimed) or $s \leq 3^{\prime}$ (primed) since $Q_{s}$ is less than 0.1 and equivalently the reduced $\chi^{2}$ per degree of freedom is significantly in excess of one.

We have investigated the extent to which the relative magnitude of the Bayes factor is driven by the greater scatter relative to a linear law, rather than by the difference of the linear fit of the Hubble constant from its 


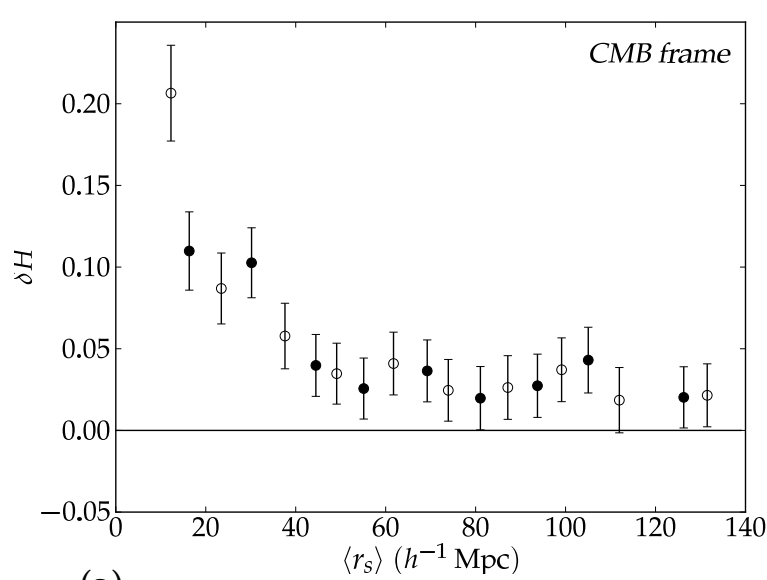

(a)

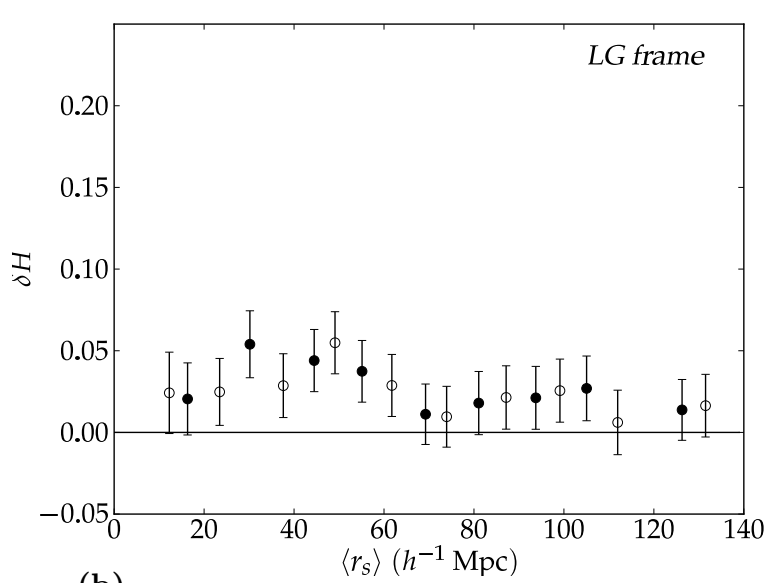

(b)

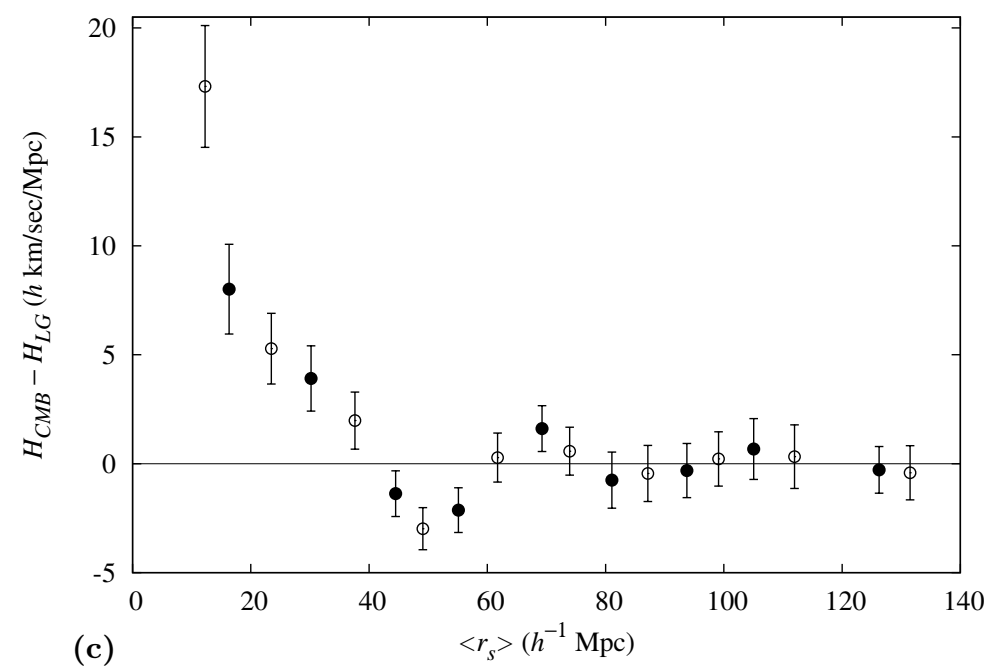

FIG. 3: Variation in the Hubble flow $\delta H_{s}=\left(H_{s}-\bar{H}_{0}\right) / \bar{H}_{0}$ in spherical shells as a function of weighted mean shell distance: (a) CMB frame; (b) LG frame. In panel (c) the difference $\left(H_{s}\right)_{\mathrm{CMB}}-\left(H_{s}\right)_{\mathrm{LG}}$ is plotted. In each case the filled data points represent the first choice of shells in Table I, and the unfilled circles the alternative second choice of shells. We have omitted the first shell from the plots since $\delta H$ is so large in the CMB frame that it is off-scale - for the first shell: with a mean weighted distance of $\left\langle r_{s}\right\rangle=5.43 h^{-1} \mathrm{Mpc}$ we have $\delta H_{\mathrm{CMB}}=0.737 \pm 0.029, \delta H_{\mathrm{LG}}=0.168 \pm 0.007$ and $H_{\mathrm{CMB}}-H_{\mathrm{LG}}=$ $56.0 \pm 8.2 \mathrm{~km} \mathrm{~s}^{-1} \mathrm{Mpc}^{-1}$.

asymptotic value. The results of this investigation are presented in Appendix $\mathrm{A}$. We find that when the data points which contribute the greatest scatter relative to a linear law are excluded, leading to a high goodness of the fit, then the Bayes factors are somewhat reduced. However, the Bayesian evidence for the greater uniformity of the LG frame Hubble flow, relative to that of the CMB frame, remains very strong.

The outer shells with $s \geq 5$ (unprimed) or $s \geq 4^{\prime}$ (primed) all have a strong goodness of fit in the full data set of Table [1. This is also true in the outermost, $r>$ $156.25 h^{-1} \mathrm{Mpc}$ shell, although it only contains 91 points. This outer shell, which is used to anchor the asymptotic value of the Hubble constant and its uncertainty, has an almost perfect goodness of fit $Q_{s}=0.999$ and a reduced $\chi^{2}$ of 0.59 per degree of freedom in both frames.
Some hints about the nature of the effects which contribute to the deviations from a uniform linear Hubble law can be discerned by comparing $\delta H_{s}$ in the shells where the deviations from uniformity become statistically significant. Perhaps the most interesting feature is that counter to the overall trend, the individual CMB frame shells $\left\{4,4^{\prime}, 5\right\}$ with mean distances in the range ${ }^{6}$ $41.0 h^{-1} \leq \bar{r}_{s} \leq 58.4 h^{-1} \mathrm{Mpc}$ have a closer to uniform $H_{s}$ than the corresponding LG frame shells. In the cumulative Bayes factor this adds a negative contribution, and reduces the overall $\ln B$ to 0.92 at $\bar{r}_{s}=49.1 h^{-1} \mathrm{Mpc}$,

\footnotetext{
${ }^{6}$ Each bound is the average of the mean distances of the shell where the CMB frame is more uniform with the mean distance of the neighbouring shell where the LG frame is more uniform.
} 


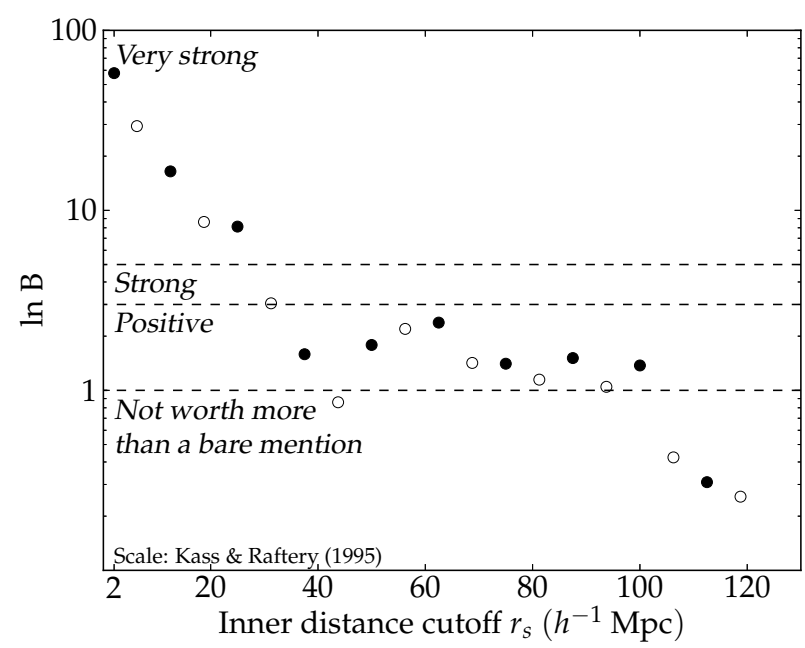

FIG. 4: The Bayes factor $\ln B$, where $B$ is the Bayes factor for the ratio of probability that the LG frame Hubble flow is uniform in the region $r>r_{s}$ outside the shell with inner radius $r_{s}$, divided by the equivalent probability for the CMB frame.

even though adjacent points have $\ln B>2$.

\section{Systematic offsets from choice of reference frame}

Another important point is to consider how the nonlinear dependence of $H_{s}$ on the individual $c z_{i}$ in the regression formula (2) can lead to systematic offsets when applying boosts. Suppose we are in a frame in which the spherically averaged variance in the Hubble flow is minimized, which of course can be a frame other than the LG or LS one. Now change reference frame by applying a uniform boost to all data points, so that

$$
c z_{i} \rightarrow c z_{i}^{\prime}=c z_{i}+v \cos \phi_{i}
$$

where $\phi_{i}$ is the angle on the sky between the data point and the boost direction. Then $\left(c z_{i}\right)^{2} \rightarrow\left(c z_{i}^{\prime}\right)^{2}=$ $c z_{i}^{2}+2 c z_{i} v \cos \phi_{i}+v^{2} \cos ^{2} \phi_{i}$ in the numerator of (2), and $c z_{i} r_{i} \rightarrow c z_{i}^{\prime} r_{i}=c z_{i} r_{i}+r_{i} \cos \left(\phi_{i}\right)$ in the denominator.

If we perform a spherically symmetric average (2) on data which is reasonably uniformly distributed over the celestial sphere $^{7}$ then on average each positive contribution of the linear terms $v \cos \phi_{i}$ in the sums in the numerator and denominator of the boosted frame $H_{s}^{\prime}$ will be counterbalanced by a negative contribution from a

\footnotetext{
7 The absence of data in the ZoA does not affect this argument, since the gaps in the data set are symmetrically distributed on opposite sides of the sky. The argument would fail if there was a significant lack of data on one side of the sky only.
}

$v \cos \phi_{j}$ on the opposite side of the sky. The linear contributions are therefore roughly self-canceling, leaving a dominant contribution to the difference

$$
\begin{aligned}
H_{s}^{\prime}-H_{s} & \sim\left(\sum_{i=1}^{N_{s}} \frac{\left(v \cos \phi_{i}\right)^{2}}{\sigma_{i}^{2}}\right)\left(\sum_{i=1}^{N_{s}} \frac{c z_{i} r_{i}}{\sigma_{i}^{2}}\right)^{-1} \\
& =\frac{\left\langle\left(v \cos \phi_{i}\right)^{2}\right\rangle_{s}}{\left\langle c z_{i} r_{i}\right\rangle_{s}} .
\end{aligned}
$$

where $\left\langle f_{i}\right\rangle_{s} \equiv\left(\sum_{i=1}^{N_{s}} f_{i} \sigma_{i}^{-2}\right)\left(\sum_{i=1}^{N_{s}} \sigma_{i}^{-2}\right)^{-1}$ is a weighted average. If we now consider (8) applied to successive shells with different values of $s$, then given a uniformly symmetrical distribution of data the weighted average $\left\langle\left(v \cos \phi_{i}\right)^{2}\right\rangle_{s} \sim \frac{1}{2} v^{2}$ in the numerator will be roughly constant from shell to shell, while putting the leading order approximation $c z_{i} \simeq H_{0} r_{i}$ in the denominator we find

$$
H_{s}^{\prime}-H_{s} \sim \frac{v^{2}}{2 H_{0}\left\langle r_{i}^{2}\right\rangle_{s}} .
$$

Consequently, for symmetrically distributed data the effect of incorporating a boost in the redshift data is an additional contribution to the spherically averaged Hubble constant which is inversely proportional to the averaged square distance. The difference between the CMB and LG frames in Fig. 3 does indeed show hints of such a dependence. Of course, the LG frame itself may incorporate such a dependence with respect to whatever frame has the minimum variance in $H_{s}$, only to a lesser extent.

We stress that by our method of analysis the effect of a spurious boost is to add a spherically symmetric, or monopole, "Hubble bubble" type variation to the Hubble relation. This feature makes the present analysis very different to the standard peculiar velocity approach, where the focus is on dipole or higher multipole variations.

We summarize the results of this section as follows. Although there are significant foreground structures which distort the spherically averaged Hubble flow in a statistically significant manner, the LG frame has a much smaller monopole Hubble flow variance than the CMB frame, counter to standard expectations. Nonetheless, there is a particular range of distances at roughly $40 h^{-1} \lesssim r \lesssim 60 h^{-1} \mathrm{Mpc}$ for which the boost to the standard CMB frame produces an apparently more uniform spherically symmetric average flow. This is the first evidence for the hypothesis we will present in Sec. V] namely that rather than being a transformation which puts us in the frame in which the Hubble flow is most uniform at our own point, the boost to the CMB frame is actually compensating for the effect of foreground structures largely associated with distance scales of order $40 h^{-1}$ to $60 h^{-1} \mathrm{Mpc}$. To better understand these structures we now consider angular averages. 


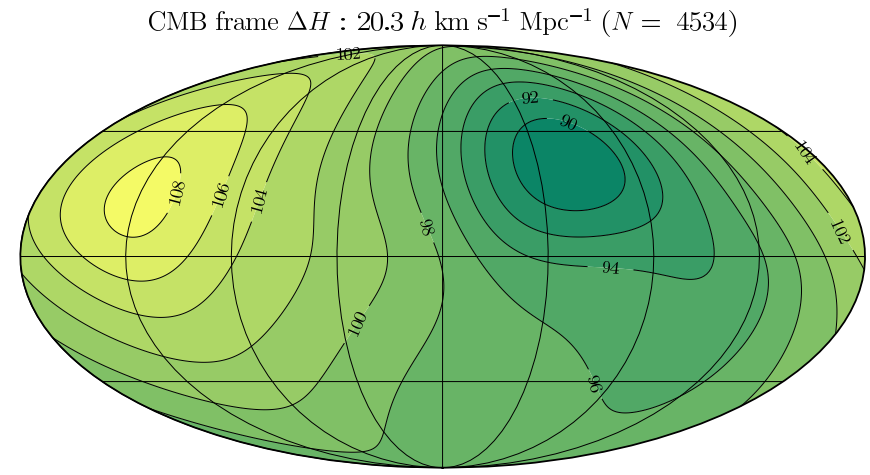

(a)

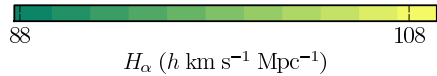

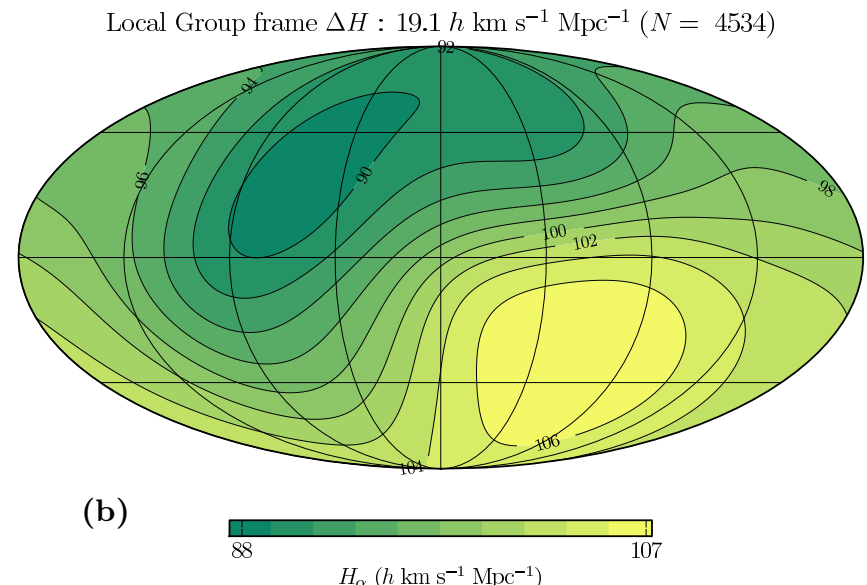

FIG. 5: Angular variance of the Hubble flow in the full COMPOSITE sample: (a) CMB rest frame; (b) LG rest frame. In all figures, the galactic longitudes $\ell=0^{\circ}, 180^{\circ}, 360^{\circ}$ are on the right edge, centre and left edge respectively.

\section{ANGULAR AVERAGES}

In order to associate variance in the Hubble flow with particular foreground structures angular information is also required. The angular variance of the Hubble flow in the same HST Key Data 41] investigated by Li and Schwarz 26] has been studied by McClure and Dyer 25] (henceforth MD07) in the CMB reference frame. McClure and Dyer [25] used all 76 points in the Key Data set, and concluded that a $13 \%$ variation in $H_{0}$ existed in the data. Once again, although individual distances in the COMPOSITE sample are noisier, the 60-fold increase in the size of data set enables a more detailed analysis.

Angular averages will in general have a complex multipole structure. The higher the order of the multipole probed, the greater the amount of data required to achieve sufficient angular resolution. Ideally one would split the sample into radial shells, as in Sec. II and perform a separate multipole analysis in each shell. Given limited data a trade-off can be made by making the width of the radial shells larger.

We find that the COMPOSITE sample has sufficient data to establish the existence of a simple dipole Hubble law in several of the shells of Sec. II with $99.99 \%$ confidence. While our main results in the present paper relate to the monopole and dipole variations, it is also necessary to perform a full multipole analysis in order to:

(i) check that multipoles higher than a dipole are sufficiently small that the dipole amplitude will not be significantly affected by leakage into higher multipoles if only a dipole Hubble law is considered;

(ii) establish a means for determining the degree of correlation between the map of angular Hubble flow variation, with all its multipoles, to the residual pure temperature dipole of the $\mathrm{CMB}$ in the rest frame of the LG or LS, as discussed in Sec. IV]

\section{A. Results of Gaussian window averages}

We have adapted the technique of MD07 to produce a series of maps of an angular average value of $c z / r$ over the sky. At each grid point on the sky, a mean $H_{\alpha}$ is calculated in which the value of $c z_{i} / r_{i}$ for each data point is weighted according to its angular separation from the grid point, with a Gaussian window function whose standard deviation, $\sigma_{\theta}$, determines the smoothing scale. The technical details are described in Appendix B. We applied the averages (B5)-(B7) both without an inverse variance (IV) weighting using (B1), and alternatively with an IV weighting using (B9). The small differences between these two methods did not affect our conclusions.

In Fig. 5 we plot the contour maps of angular Hubble flow variance produced using the whole COMPOSITE data set in a single sphere, in both the CMB and LG rest frames. This allows a direct comparison to MD07 who considered only 76 points in a single sphere in the CMB rest frame.

To study radial variations one can simultaneously break the sample into independent shells as in Sec. II. There is enough data in the COMPOSITE sample to reliably establish a quadrupole and perhaps higher order multipoles in many of the shells of Table [ However, our first aim is to determine the gross features of the relative angular variation. We will therefore perform the most simple of radial separations: we divide the data into an inner $\left(r<r_{o}\right)$ and an outer $\left(r>r_{o}\right)$ sphere, with a boundary $r_{o}$ which we vary, and reperform the Gaussian window averages.

We show a subset of the resulting sky maps in Figs. 6 


\section{CMB frame}

In
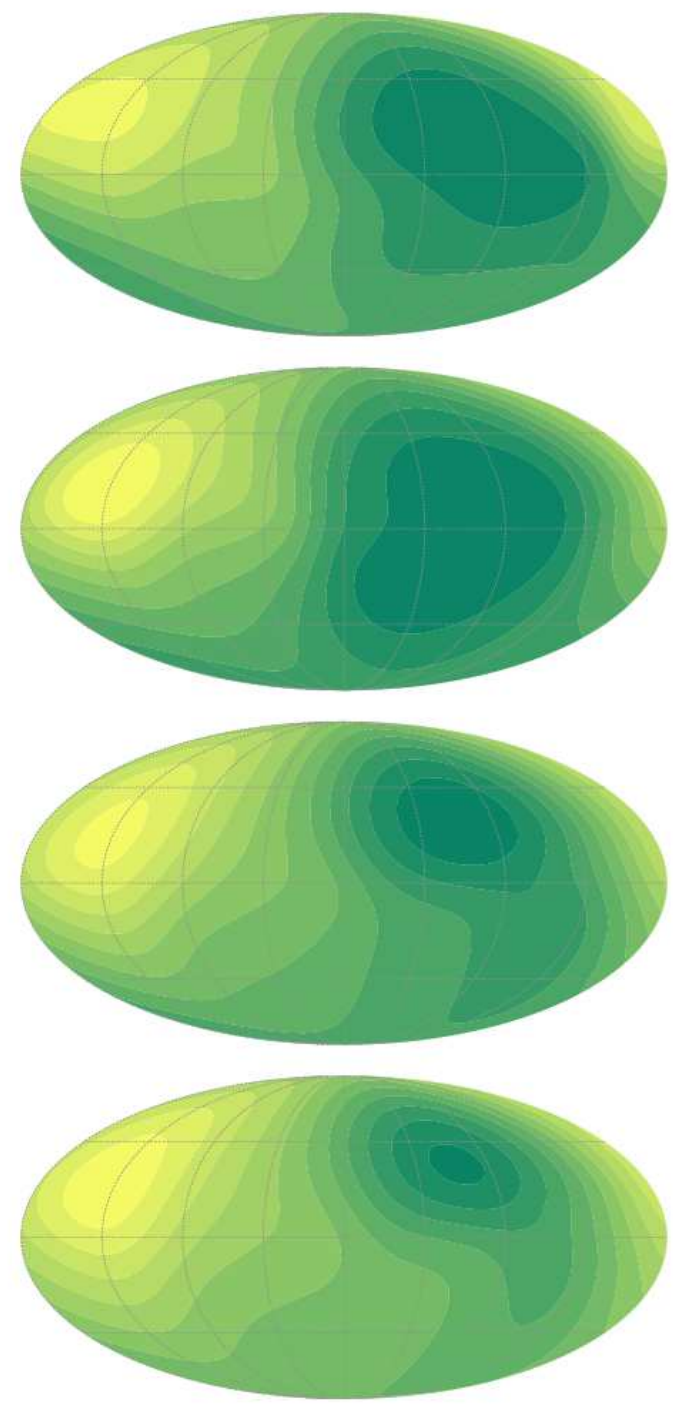

Out

$12.5 / \mathrm{h} \mathrm{Mpc}$

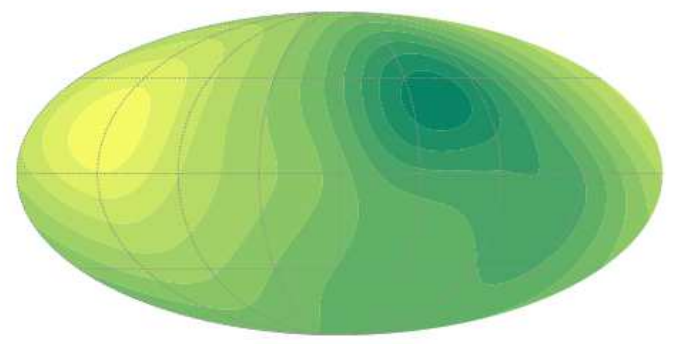

$20 / \mathrm{h} \mathrm{Mpc}$

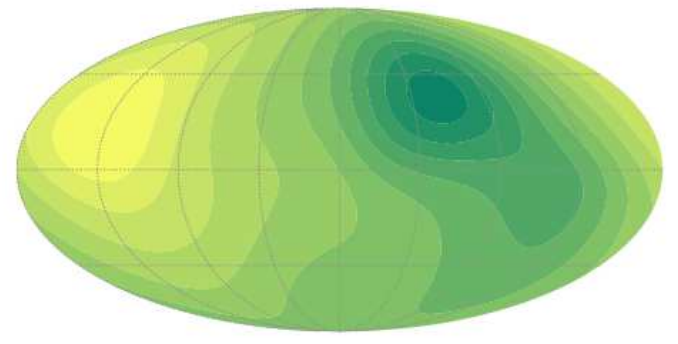

$40 / \mathrm{h} \mathrm{Mpc}$

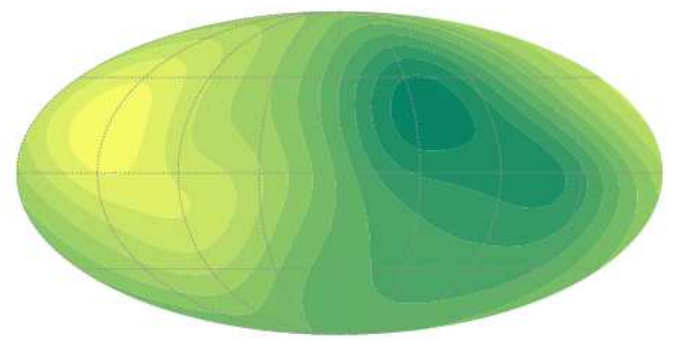

$60 / \mathrm{h} \mathrm{Mpc}$

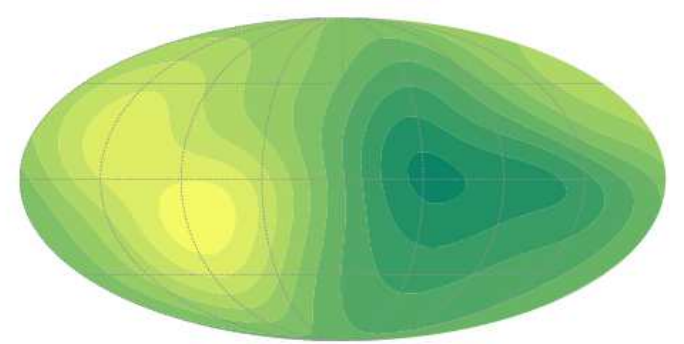

FIG. 6: Angular variance in the Hubble flow in the CMB rest frame for inner $\left(r<r_{o}\right.$ left panel) and outer $\left(r>r_{o}\right.$ right panel) spheres as $r_{o}$ is varied over the values $12.5,20,40$ and $60 \mathrm{~h}^{-1} \mathrm{Mpc}$. In all figures, the galactic longitudes $\ell=0^{\circ}, 180^{\circ}, 360^{\circ}$ are on the right edge, centre and left edge respectively.

and 7 for the CMB and LG rest frames, with the boundary between the inner and outer spheres taking the values $12.5 h^{-1}, 20 h^{-1}, 40 h^{-1}$ and $60 h^{-1} \mathrm{Mpc}$.

The maps are of course not entirely independent, as there is overlap of data between the outer shells for small $r_{o}$ and the inner shells of maps with larger $r_{o}$. The extent of overlap of sources, and their angular distribution, can be determined roughly from the numbers given in Table I and in Fig. 2, where points within individual shells are shown. Working with maps which are not independent shows how power in the dipole is transferred from the outer to inner sphere as $r_{o}$ is varied.

The first observation we make is that although both frames reveal a dipole structure, the nature of the dipole has important differences between the two frames. In the CMB frame the difference between the inner and outer spheres is not very strong. In the outer sphere the two poles migrate from being both in the northern hemisphere in the $r>12.5 h^{-1} \mathrm{Mpc}$ map to both being close to the galactic equator in the $r>60 h^{-1} \mathrm{Mpc}$ map, while the poles in the corresponding interior spheres become localized to the northern hemisphere. However, the 


\section{Local Group frame}
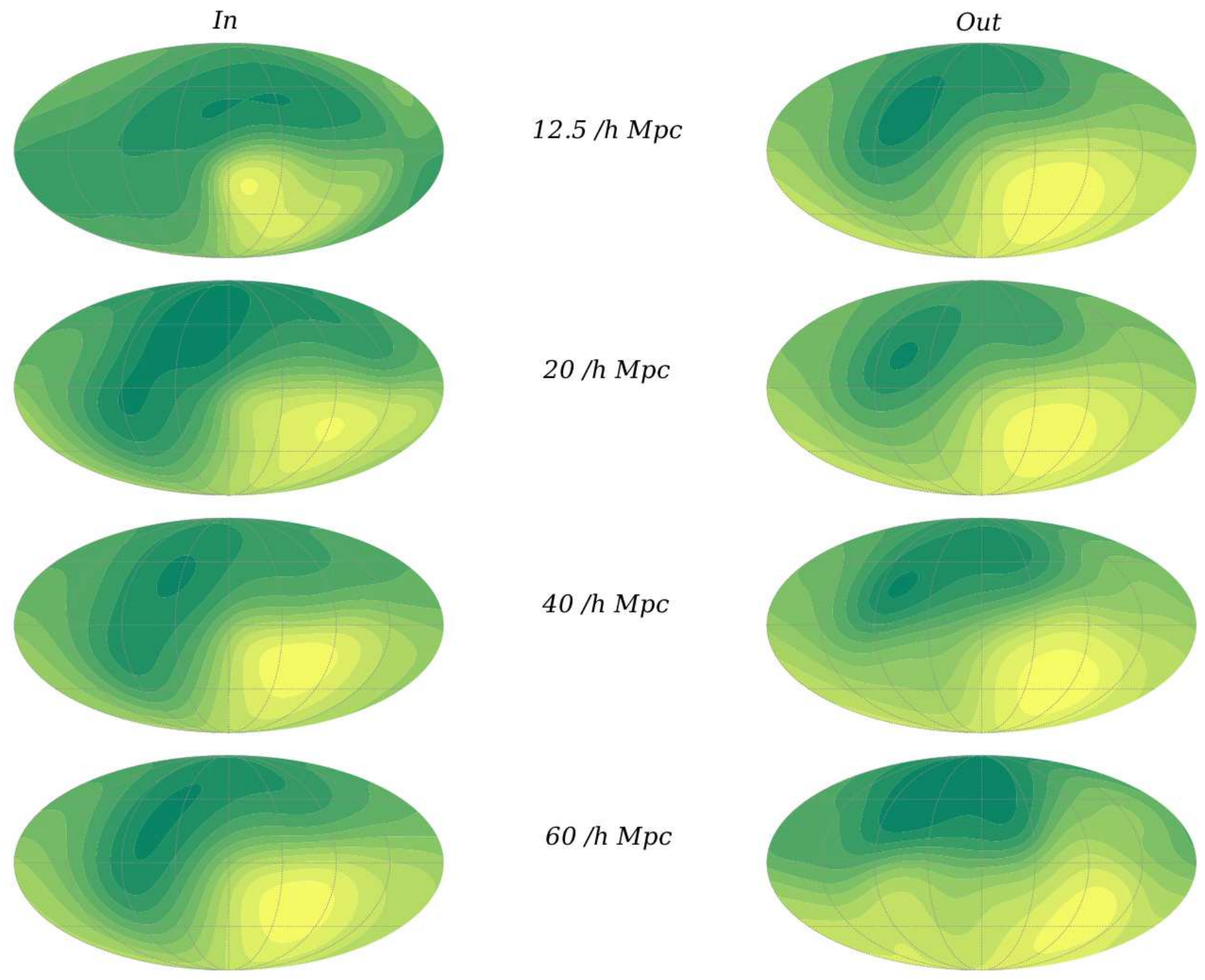

FIG. 7: Angular variance in the Hubble flow in the LG rest frame for inner $\left(r<r_{o}\right.$ left panel $)$ and outer $\left(r>r_{o}\right.$ right panel) spheres as $r_{o}$ is varied over the values $12.5,20,40$ and $60 h^{-1} \mathrm{Mpc}$. In all figures, the galactic longitudes $\ell=0^{\circ}, 180^{\circ}, 360^{\circ}$ are on the right edge, centre and left edge respectively.

strength of the dipole feature does not vary significantly between the inner and outer spheres, nor with the variation of the boundary $r_{o}$ between the inner and outer spheres. The fact that both poles are in the northern hemisphere in most of the CMB frame plots also means of course that the dipole is less strong than for example in the $r>12.5 h^{-1} \mathrm{Mpc}$ LG frame map, for which the poles are closer to $180^{\circ}$ apart.

By contrast to the CMB frame, in the LG frame there is a significant radial dependence to the Hubble variance dipole evident in Fig. 7. With the division set at $r_{o}=$
$12.5 h^{-1} \mathrm{Mpc}$ there is very strong dipole feature in the outer $r>12.5 h^{-1} \mathrm{Mpc}$ sphere, which is stronger than in the full sample map of Fig. 5 . By contrast, within the inner $12.5 h^{-1} \mathrm{Mpc}$ sphere any dipole signature is masked by other multipoles which appear equally as strong.

As the division scale $r_{o}$ is increased the relative power in the dipole in the inner sphere in the LG frame maps increases substantially, so that by the time we reach $r_{o}=$ $60 h^{-1} \mathrm{Mpc}$ the inner sphere shows a dipole almost as distinct as the outer sphere of the $r_{o}=12.5 h^{-1} \mathrm{Mpc}$ map. At the same time the dipole in the outer $r>60 h^{-1} \mathrm{Mpc}$ 


\begin{tabular}{|c|c|c|c|c|c|c|c|c|c|c|c|c|}
\hline$r<r_{o}\left(h^{-1} \mathrm{Mpc}\right)$ & & $<12.5$ & $<15$ & $<20$ & $<30$ & $<40$ & $<50$ & $<60$ & $<70$ & $<80$ & $<90$ & $<100$ \\
\hline $\mathrm{CMB} C_{2} / C_{1}$ & & 0.123 & 0.061 & 0.044 & 0.098 & 0.136 & 0.191 & 0.187 & 0.167 & 0.141 & 0.134 & 0.120 \\
\hline $\mathrm{CMB} C_{3} / C_{1}$ & & 0.010 & 0.011 & 0.007 & 0.003 & 0.005 & 0.010 & 0.009 & 0.011 & 0.011 & 0.012 & 0.012 \\
\hline $\mathrm{LG} C_{2} / C_{1}$ & & 0.653 & 0.179 & 0.123 & 0.135 & 0.116 & 0.103 & 0.104 & 0.103 & 0.092 & 0.089 & 0.085 \\
\hline $\mathrm{LG} C_{3} / C_{1}$ & & 0.067 & 0.018 & 0.008 & 0.005 & 0.008 & 0.006 & 0.007 & 0.009 & 0.011 & 0.011 & 0.011 \\
\hline $\mathrm{LS} C_{2} / C_{1}$ & & 0.861 & 0.197 & 0.133 & 0.146 & 0.124 & 0.112 & 0.113 & 0.112 & 0.101 & 0.097 & 0.093 \\
\hline $\mathrm{LS} C_{3} / C_{1}$ & & 0.068 & 0.015 & 0.006 & 0.005 & 0.007 & 0.006 & 0.007 & 0.009 & 0.011 & 0.012 & 0.011 \\
\hline$r>r_{o}\left(h^{-1} \mathrm{Mpc}\right)$ & $>2$ & $>12.5$ & $>15$ & $>20$ & $>30$ & $>40$ & $>50$ & $>60$ & $>70$ & $>80$ & $>90$ & $>100$ \\
\hline $\mathrm{CMB} C_{2} / C_{1}$ & 0.102 & 0.096 & 0.115 & 0.124 & 0.073 & 0.038 & 0.023 & 0.041 & 0.093 & 0.093 & 0.090 & 0.327 \\
\hline $\mathrm{CMB} C_{3} / C_{1}$ & 0.010 & 0.009 & 0.013 & 0.015 & 0.017 & 0.009 & 0.007 & 0.011 & 0.018 & 0.078 & 0.069 & 0.076 \\
\hline $\mathrm{LG} C_{2} / C_{1}$ & 0.072 & 0.061 & 0.064 & 0.064 & 0.053 & 0.042 & 0.032 & 0.045 & 0.068 & 0.077 & 0.066 & 0.151 \\
\hline $\mathrm{LG} C_{3} / C_{1}$ & 0.010 & 0.009 & 0.012 & 0.014 & 0.019 & 0.013 & 0.013 & 0.014 & 0.010 & 0.048 & 0.051 & 0.016 \\
\hline $\mathrm{LS} C_{2} / C_{1}$ & 0.079 & 0.065 & 0.068 & 0.068 & 0.054 & 0.044 & 0.033 & 0.048 & 0.074 & 0.079 & 0.070 & 0.162 \\
\hline $\mathrm{LS} C_{3} / C_{1}$ & 0.010 & 0.009 & 0.012 & 0.014 & 0.019 & 0.013 & 0.013 & 0.014 & 0.011 & 0.053 & 0.062 & 0.020 \\
\hline
\end{tabular}

TABLE II: Ratios $C_{2} / C_{1}, C_{3} / C_{1}$ of quadrupole/dipole and octupole/dipole for the multipoles of the angular Hubble variance maps in the CMB, LG and LS frames, using (B2) with no IV variance weighting. In each case the multipole ratios are computed inside $\left(r<r_{o}\right)$ and outside $\left(r>r_{o}\right)$ a bounding shell.

map becomes less distinct. This is consistent with our finding in the previous section that the structures principally responsible for the Hubble flow variance lie within $r<65 h^{-1} \mathrm{Mpc}$. This conclusion will be confirmed by an independent analysis of the data in Sec. IIIB below.

We remark that the dipole feature in the LG frame can be seen by eye in the colour coded peculiar velocities relative to $H_{s}$ in each shell, as shown in Fig. 2, in shells 2 and 3 , which cover the range $12.5 h^{-1}<r<37.5 h^{-1} \mathrm{Mpc}$ there is a clear concentration of negative peculiar velocities (blue) in the upper left quadrant and positive peculiar velocities (red) in the lower right quadrant, which correlate with the dipole structure in Fig. 7. These concentrations of peculiar velocities become more and more diluted by the contributions of peculiar velocities of the opposite sign in shells 4 and 5 , where $37.5 h^{-1}<r<$ $62.5 h^{-1} \mathrm{Mpc}$. In shells with $r>62.5 h^{-1} \mathrm{Mpc}$ the areas previously associated with the dipole feature contain similar numbers of positive and negative peculiar velocities.

The fact that the CMB frame dipole shows far less variation than the LG frame dipole as $r_{o}$ is varied is consistent with the hypothesis that it is not directly associated with the structures defining the Hubble flow variance but is rather due to an overall systematic, namely the relative boost to the CMB frame, as discussed in Sec. IIC.

The above statements are of course made from a simple inspection of the sky maps by eye. However, the statements can be quantified by performing a spectral analysis on the sky maps. To this end we digitized the contour maps into 1 square degree regions and performed a multipole analysis using HEALPIX $^{8}$ to determine the relative power in the $C_{\ell}$ coefficients. On account of the Gaussian window averaging there is aliasing at the $25^{\circ}$

\footnotetext{
${ }^{8}$ http://healpix.jpl.nasa.gov/ 47]
}

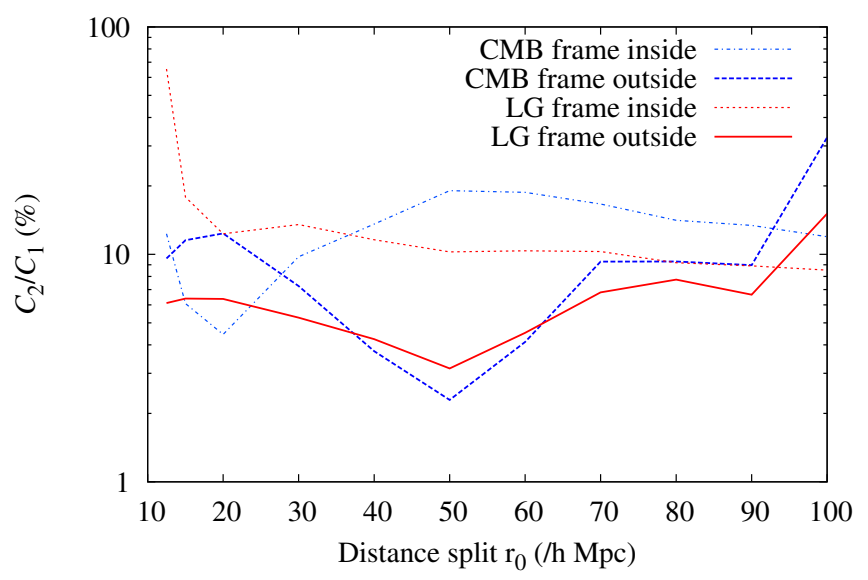

FIG. 8: Ratios $C_{2} / C_{1}$, of quadrupole to dipole, on inner $(r<$ $\left.r_{o}\right)$ and outer $\left(r>r_{o}\right)$ spheres, as $r_{o}$ is varied in the CMB and LG rest frames.

scale, and information for the high multipoles is not reliable. However, since multipoles with $\ell \geq 4$ are very much suppressed a good measure of the significance of the dipole can be estimated by determining the quadrupole to dipole ratio, $C_{2} / C_{1}$, and octupole to dipole ratio, $C_{3} / C_{1}$, as listed in Table $\amalg$ in the inner and outer spheres as the boundary, $r_{o}$, is varied in the CMB, LG and LS frames. The inner and outer $C_{2} / C_{1}$ ratios are also illustrated graphically for the CMB and LG frames in Fig. 8 ,

In the LG frame $C_{2} / C_{1}=0.061$ in the outer $r>$ $12.5 h^{-1} \mathrm{Mpc}$ sphere, representing a small quadrupole relative to dipole while $C_{2} / C_{1}=0.653$ in the corresponding inner sphere representing a quadrupole roughly comparable to the dipole. By contrast in the CMB frame with $r_{o}=12.5 h^{-1} \mathrm{Mpc}$, the respective ratios are $C_{2} / C_{1}=0.096(0.123)$ in the outer (inner) sphere, indicating a dipole which is similar in both spheres, and less 
clearly defined than in the outer LG frame.

In the inner sphere the ratio $C_{2} / C_{1}$ in the $\mathrm{LG}$ frame drops substantially for $r_{o} \geq 30 h^{-1} \mathrm{Mpc}$, and maintains a value in the range $0.09-0.12$ when $40 h^{-1} \leq r_{o} \leq$ $90 h^{-1} \mathrm{Mpc}$. This is of course higher than the same ratio in the outer sphere; but the inner sphere value includes in every case a contribution from the innermost shell in which the dipole and quadrupole are comparable.

The outer dipole is stronger in the LG frame than the CMB frame except for the values $40 h^{-1} \lesssim r \lesssim 60 h^{-1} \mathrm{Mpc}$, for which the situation is reversed. The outer $\mathrm{CMB}$ dipole is strongest for $r>50 h^{-1} \mathrm{Mpc}$, when the quadrupole/dipole ratio drops to a minimum $C_{2} / C_{1}=0.023$ as compared to $C_{2} / C_{1}=0.032$ in the $\mathrm{LG}$ frame. However, when $r_{o} \geq 70 h^{-1} \mathrm{Mpc}$ the outer CMB dipole becomes less distinct again. As we saw earlier the variance in the spherically averaged Hubble flow was less in the CMB frame in the range $35 h^{-1} \lesssim r \lesssim 60 h^{-1} \mathrm{Mpc}$. It appears that the boost to the $\mathrm{CMB}$ frame is also having the effect of making the angular variance of the CMB frame Hubble flow more dipole-like over this particular radial range.

Table III shows that the quadrupole and higher order multipoles are at least an order of magnitude smaller than the dipole in the range $15 \lesssim r \lesssim 65 h^{-1} \mathrm{Mpc}$, and therefore a simple dipole law can be reliably used in this range. However, for $r_{o}>90 h^{-1} \mathrm{Mpc}$ the ratio $C_{2} / C_{1}$ increases substantially, so caution should be exercised about fitting a simple dipole in the outermost shells.

\section{B. Dipole law averages in radial shells}

It is difficult to provide statistical bounds on the angular orientation and magnitude of the Hubble flow variance dipole with Gaussian window averaging. However, a completely independent analysis can be made by fitting the raw data to a simple linear dipole law

$$
\frac{c z}{r}=H_{d}+\beta \cos \phi
$$

for the LG and CMB rest frames, where in each case $\phi$ is the angle on the sky between each galaxy and the direction $\left(\ell_{d}, b_{d}\right)$ which defines the best fit dipole axis. This method is similar to that used in Fig. 9 of Ref. [48] or Fig. 8 of Ref. [49].

In each case we determine the four parameters $H_{d}$, $\beta, \ell_{d}$ and $b_{d}$ by a least squares fit of $H_{d}$ and the linear parameters $\beta_{x}=\beta \cos \ell_{d} \cos b_{d}, \beta_{y}=\beta \cos \ell_{d} \sin b_{d}$, and $\beta_{z}=\beta \sin b_{d}$. Details are given in Appendix C]. The results of the analysis for the same independent shells chosen in Table [I are tabulated in Table [VII] and the corresponding dipole amplitudes are plotted in Fig. 9 Here statistical $1 \sigma$ uncertainties are shown.

Monte Carlo simulations, discussed in Appendix D, establish that random reshuffles of the angular data in each shell are consistent with zero dipole. We also determine a

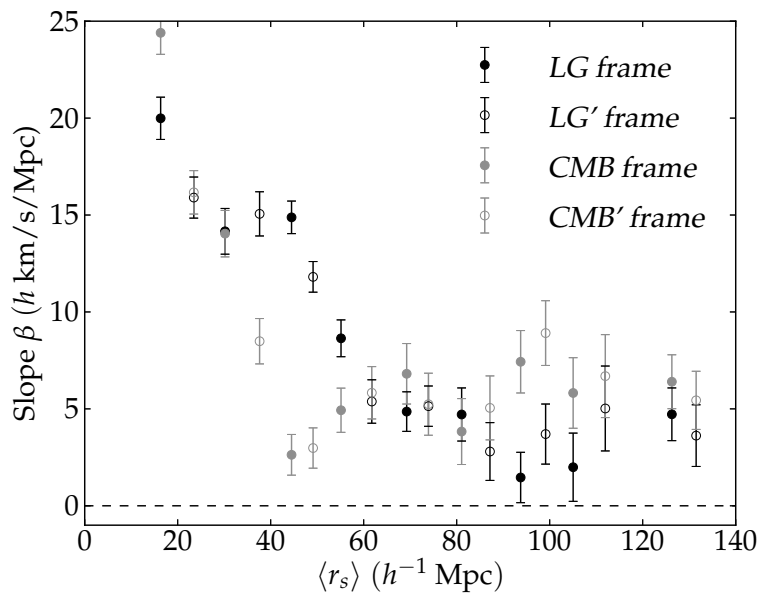

FIG. 9: The slope $\beta$ of the linear dipole relation $c z / r=H_{d}+$ $\beta \cos \phi$, as given in Table VII, is plotted by shell in the CMB and LG rest frames. The filled (unfilled) circles correspond to the unprimed (primed) shells.

probability in excess of random of the dipole law providing a better fit, and consequently a statistical confidence that the dipole in each shell is indeed non-zero.

We see from Fig. 9 that the range of radial shells over which the CMB frame provides a better fit to the monopole Hubble law than the LG frame also coincides with a dramatic difference in changes to the dipole in the two frames. The magnitudes of the dipoles in the two frames coincide in shell 3 with $\bar{r}_{3}=30.2 h^{-1} \mathrm{Mpc}$, taking the values $\beta_{\mathrm{CMB}}=(14.0 \pm 1.2) h \mathrm{kms}^{-1} \mathrm{Mpc}^{-1}$ and $\beta_{\mathrm{LG}}=(14.2 \pm$ 1.2) $h \mathrm{~km} \mathrm{~s}^{-1} \mathrm{Mpc}^{-1}$ respectively. They also coincide in shell $5^{\prime}$, with $\bar{r}_{5^{\prime}}=61.7 h^{-1} \mathrm{Mpc}$, where they take the reduced values $\beta_{\mathrm{CMB}}=(5.8 \pm 1.3) h \mathrm{~km} \mathrm{~s}^{-1} \mathrm{Mpc}^{-1}$ and $\beta_{\mathrm{LG}}=(5.4 \pm 1.2) h \mathrm{~km} \mathrm{~s}^{-1} \mathrm{Mpc}^{-1}$. However, the dipoles exhibit very different behaviour for the shells in between. In particular, the $\mathrm{CMB}$ dipole magnitude reaches a minimum of $\beta=(2.6 \pm 0.6) h \mathrm{~km} \mathrm{~s}^{-1} \mathrm{Mpc}^{-1}$ (close to zero dipole) in shell 4 , for which $\bar{r}_{4}=44.5 h^{-1} \mathrm{Mpc}$, whereas for the LG frame $\beta=(14.9 \pm 0.8) h \mathrm{~km} \mathrm{~s}^{-1} \mathrm{Mpc}^{-1}$ in the same shell. The CMB frame dipole then increases while the LG frame dipole decreases so the two take similar values at $\bar{r}_{5^{\prime}}=61.7 h^{-1} \mathrm{Mpc}$. The dipole directions in each frame are strongly consistent in shells 4 to 6 in the range $37.5 h^{-1} \leq r \leq 62.5 h^{-1} \mathrm{Mpc}$.

The analysis of Appendix $\mathrm{D}$ shows that in shells 3 to 5' our principal conclusions above concerning the relative magnitudes of the dipoles are supported to the level of at least $99.9 \%$ confidence. We therefore have a statistically robust justification for the conclusion that the boost from the $L G$ to $C M B$ frame is compensating for structures in the range $30 h^{-1} \lesssim r \lesssim 62 h^{-1} \mathrm{Mpc}$.

There are some further changes to the dipoles in the outer shells. A small residual dipole of amplitude $\sim 5 \mathrm{kms}^{-1} \mathrm{Mpc}^{-1}$ and roughly consistent direction is maintained in the LG frame in shells 5 ' to 7 , at the $90 \%$ 
confidence level. Beyond this scale there is no significant LG frame dipole, with the exception of shell 10. By contrast a dipole is found with at least $90 \%$ confidence level in all shells 5' to 10 the CMB frame, and with more than $95 \%$ confidence the shells 8 and 9 which lack a significant LG frame dipole.

Although there appears to be a significant dipole in both frames in shell 10, we note from Fig. 8 that the quadrupole to dipole ratio in the Gaussian window averages begins to increase significantly at $r_{o} \sim 100 h^{-1} \mathrm{Mpc}$. Given a strong quadrupole, and the fact that the LG dipole axis is roughly orthogonal to the largest mass concentration in shell 10 - the Shapley Concentration - we should be careful not to draw strong conclusions from the fit of a simple dipole law (10) in the outer shell, as the magnitude may change once higher multipoles are included. The COMPOSITE sample does not have enough data in shell 10 to constrain the quadrupole; considerably more data is required.

\section{Smoothed dipole law variation}

The analysis of the previous subsection provides the strongest direct evidence that there is a correlation between the structures responsible for both the monopole and dipole variations of the Hubble law in the range $30 h^{-1} \lesssim r \lesssim 62 h^{-1} \mathrm{Mpc}$. However, to make contact with the result of analyses in the peculiar velocity formalism, and with the Gaussian window averages, it is also useful to consider the results of a dipole law fit on all data outside (inside) a sphere $r>r_{o}\left(r \leq r_{o}\right)$.

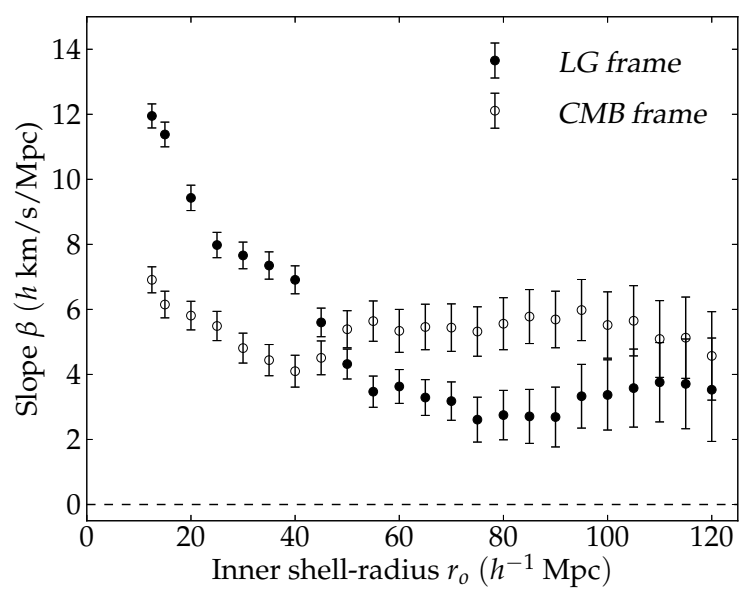

FIG. 10: All data in a sphere $r>r_{o}$ is fit to the linear dipole relation (10) in the CMB and LG rest frames. We plot the slope, $\beta$, of this relation as a function of the radius $r_{o}$ outside which the data is included. Open data points represent fits for the CMB rest frame and solid data points fits for the LG rest frame.

The results averaged in an outer sphere are tabulated in Table VIII of Appendix [C] and the corresponding dipole magnitudes are plotted in Fig.10. In the LG frame there is a very strong dipole with magnitude $\beta=11.4 \pm$ $0.4 \mathrm{~km} \mathrm{~s}^{-1} \mathrm{Mpc}^{-1}$ for $r_{o}=15 h^{-1} \mathrm{Mpc}$, which decreases to a typical value $\beta \sim 3.5 \mathrm{~km} \mathrm{~s}^{-1} \mathrm{Mpc}^{-1}$ for the largest values of $r_{o}$ in Table VIII, which is of order $3 \sigma$ different from zero. If we consider only those cases with goodness of fit $Q>0.1$ then the most abrupt decrease in $\beta$ occurs in the range $40 h^{-1} \leq r_{o} \leq 55 h^{-1} \mathrm{Mpc}$ where $\beta$ decreases from $6.9 \pm 0.4 \mathrm{~km} \mathrm{~s}^{-1} \mathrm{Mpc}^{-1}$ to $3.5 \pm 0.5 \mathrm{~km} \mathrm{~s}^{-1} \mathrm{Mpc}^{-1}$.

The diminishing of the LG frame dipole at is consistent with the results of the previous two subsections. Furthermore the angular position of the dipole for $20 h^{-1} \lesssim r_{o} \lesssim 45 h^{-1} \mathrm{Mpc}$ is consistently in the range $\left(\ell_{d}, b_{d}\right)=\left(83^{\circ} \pm 6^{\circ},-39^{\circ} \pm 3^{\circ}\right)$ while the dipole is strong, but the angular position then wanders once its magnitude is reduced to residual levels. For $r_{o} \gtrsim 80 h^{-1} \mathrm{Mpc}$ the typical position of the residual dipole differs from that of the inner dipole by $80^{\circ}-100^{\circ}$ in galactic longitude. The direction coincides with that of the data in shell 10, which contains the only significant dipole in the outer regions.

By contrast, the magnitude of $\beta$ is initially smaller in the $\mathrm{CMB}$ frame for small values of $r_{o}$, with a value $\beta=6.1 \pm 0.4 \mathrm{kms}^{-1} \mathrm{Mpc}^{-1}$ at $15 h^{-1} \mathrm{Mpc}$ which decreases somewhat to $\beta=4.1 \pm 0.5$ at $r_{o}=40 h^{-1} \mathrm{Mpc}$. However, $\beta$ then increases to $5.6 \pm 0.6 \mathrm{~km} \mathrm{~s}^{-1} \mathrm{Mpc}^{-1}$ at $r_{o}=55 h^{-1} \mathrm{Mpc}$, and for larger $r_{o}$ its value remains roughly constant at this level, which is of order $4 \sigma-$ $7 \sigma$ different from zero. Furthermore, over the entire range $15 h^{-1} \leq r \leq 120 h^{-1} \mathrm{Mpc}$ the direction $\left(\ell_{d}, b_{d}\right)$ remains within $1 \sigma$ of the "dark flow" direction $(\ell, b)=$ $\left(296^{\circ}, 14^{\circ}\right) \pm 13^{\circ}$ found by Kashlinsky et al. [49] for $\mathrm{X}-$ ray clusters in the range ${ }^{9} 0.05 \leq z \leq 0.3$. It also within $1 \sigma$ of the bulk flow direction $(\ell, b)=\left(287^{\circ} \pm 9^{\circ}, 8^{\circ} \pm 6^{\circ}\right)$ found by Watkins, Feldman and Hudson [18] for all values $20 h^{-1} \leq r_{o} \leq 115 h^{-1} \mathrm{Mpc}$. For the largest values of $r_{o}$ in Table VIII the direction ${ }^{10}$ remains consistent with the bulk flow direction $(\ell, b)=\left(319^{\circ} \pm 18^{\circ}, 7^{\circ} \pm 14^{\circ}\right)$ of Turnbull et al. [24]. This suggests that as far as the dipole direction is concerned the slight difference between the results of WFH09, FWH10 and Ref. [24] is accounted for by the latter study having a greater mean depth.

For the largest values of $r_{o}$ in Table VIII the CMB frame dipole direction is also consistent with some other cosmic dipoles that have been observed: a dipole in the fine structure constant [50]-[52] and the maximum temperature asymmetry [53].

A comparison of Fig. 9] and Fig. 10 shows that

${ }^{9}$ When restricted to the larger scales, $0.12 \leq z \leq 0.3$, the dark flow direction is $\left(267^{\circ}, 34^{\circ}\right) \pm 15^{\circ}$, which coincides with the direction of the residual CMB dipole in the LG frame.

${ }^{10}$ In the outermost shell 11 of Table VII with $r>156.25 h^{-1} \mathrm{Mpc}$, the uncertainty in the dipole position in the CMB frame is essentially the whole sky, meaning that there is not enough data to constrain the dipole in this range, as is confirmed by the analysis of section $D$ In the $L G$ frame the magnitude of the dipole in this shell is just $1.2 \sigma$ from $\beta=0$. 
analysing the data only in terms of smoothed dipoles on large scales - as is implicit in the peculiar velocities approach - can hide much information. In particular, the residual LG frame smoothed dipole at $r \gtrsim 80 h^{-1} \mathrm{Mpc}$ is accounted for by a feature in shell 10, whereas there is no significant LG frame dipole in shells 8 and 9. By contrast there is a CMB frame dipole in the shell by shell analysis; thus its nature is different, consistent with our hypothesis of Sec. II C.

\section{Identification of Hubble variance with particular structures}

A large dipole structure in the Hubble flow across the sky is consistent with a foreground density gradient leading to concentrations of more rapidly expanding void regions in one sector of the sky, and less rapidly expanding wall regions in the opposite sector. A detailed understanding of the structures within $30 h^{-1} \mathrm{Mpc}$ may be gained from the work of Ref. [10], and a viewing of the associated video ${ }^{11}$ is particularly instructive. Sky maps of structures on larger scales are given by Erdoğdu et al. [54. using the 2 Micron All-Sky Redshift Survey (2MASS).

The distinctive feature of the location of our galaxy is that it is in a thin filamentary sheet, the LS, which defines the supergalactic $(X, Y)$ plane, on the edge of a Local Void of at least $30 h^{-1} \mathrm{Mpc}$ diameter. While large void regions dominate one side of the sky, wall regions dominate the other side of the sky with the superclusters of Centaurus, Hydra and Norma being particularly prominent. Our Local Sheet and nearby filamentary sheets such as the Leo Spur are of modest density. The Virgo Cluster appears to be the closest region of the thick section of a dense nearby wall; however, it lies almost in the supergalactic plane of the LS, rather than along the axis which defines the greatest density contrast between the nearby voids and walls.

In this particular situation our galaxy is neither in the middle of one of the largest typical voids of $30 h^{-1} \mathrm{Mpc}$ diameter, nor is it in the middle of one of the thick wall regions. Rather it is in the transition zone between void and modest wall structures, close to the edge of both. In this circumstance the observed dipole pattern of Hubble flow variance might be expected to be the dominant one. Since the spatial width of typical walls is generally smaller than the diameter of the largest typical voids, observers located in the middle of a thick wall region with extent in their $(X, Y)$ plane, with larger typical voids some way off and equidistant along their $\pm Z$ axes, might in contrast to our situation expect to see a more dominant quadrupole Hubble variance.

The angular extent of various structures must also be important in determining how close the pattern of Hub-

11 http://ifa.hawaii.edu/ tully/pecv_12min_sound_qt.mov ble flow variance is to a dipole. A pure step function contains many higher multipoles, so a simple division of the whole sky into two hemispheres of uniform faster and slower expansion would contain many higher multipoles. Since voids have a purer ellipsoidal geometry than walls, in terms of defining the relevant angular scales it is the voids which will more clearly delineate the dipole density gradient.

Some estimate of the angular scales of the nearest voids can be obtained from the work of Tully et al. [10]. However, since the distances of many galaxies in their survey are not known Tully et al. [10] present their diagrams in redshift space, which are subject to redshift space distortions as large as the Hubble flow variance that we are endeavouring to measure.

Our own Local Void comprises three separate smaller volumes: the Inner Local Void, and the Local Voids North and South, which are separated by filamentary thin wall structures [10]. Here "north" and "south" refer to directions orthogonal to the LG in supergalactic coordinates; and since the plane of our galaxy is roughly orthogonal to that of the LS, this means that supergalactic "north" and "south" indicate directions principally along the galactic longitude axis relative to the supergalactic north pole at $\ell=47.37^{\circ}, b=6.32^{\circ}$.

The Inner Local Void, which is ellipsoidal with its major axis roughly parallel to the Local Sheet, is the structure that covers the largest fraction of the sky in the Local Void complex. From Fig. 10 of [10] we estimate that it covers at least $40-60 \%$ of one hemisphere, given the uncertainties of redshift space distortions. In any case we expect it to be too large a fraction of the sky to give a pure dipole. This is confirmed by splitting the inner and outer shells at $r_{o}=12.5 h^{-1} \mathrm{Mpc}$, since the inner shell should just exclude the Inner Local Void while retaining the Local Voids North and South. As shown in the first panel of Fig. 7 and in Table II, the inner sphere in these cases has similar power in the quadrupole.

The dipole axis appears to be principally defined by structures within the range $30 h^{-1}-62 h^{-1} \mathrm{Mpc}$, which lies beyond the scales explicitly identified by Tully et al. 10]. However, using the 2MASS survey Erdoğdu et al. 54] have reconstructed the density field in shells every $20 h^{-1} \mathrm{Mpc}$ out to $160 h^{-1} \mathrm{Mpc}$. To define a dipole, rather than simply locating the largest overdensity or underdensity, one must find an axis where the integrated density gradient, including foregrounds, is maximized. If we compare $^{12}$ the results of Sec. IIIB and B 1 to Fig. 3 of Ref. 54] we see that the minimum Hubble variance pole coincides with the near side of the Centaurus-Hydra Wall on one side of the sky and the maximum Hubble variance pole coincides with the Andromeda Void on the opposite side of the sky. Our axis to the Andromeda Void passes

\footnotetext{
12 Note that Ref. [54] places galactic longitude $\ell=0^{\circ}$ in the centre
} of their skymaps, rather than to the right edge as we do. 
through the Inner Local Void and the edge of the Local Void North ${ }^{13}$.

The centre of the Hydra Supercluster is at $r=(34.9 \pm$ 2.5) $h^{-1} \mathrm{Mpc},(\ell, b)=\left(269.6^{\circ}, 26.5^{\circ}\right)$, and the centre of the Centaurus Supercluster is at $(31.5 \pm 2.6) h^{-1} \mathrm{Mpc}$, $(\ell, b)=\left(302.4^{\circ}, 21.6^{\circ}\right)$. These radial distances are close to the radial scale at which the CMB frame Hubble flow begins to be more uniform than the LG one. In the next sky map plotted in Ref. [54], at $r=40 h^{-1} \mathrm{Mpc}$, the Hydra Supercluster remains very dense near the Hubble variance angular minimum while on the opposite side of the sky the Andromeda void has begun to close up, but an adjacent void complex Cygnus-Aquarius has opened up, maintaining the dipole density gradient. By Fig. 5 of Ref. [54] at $r=60 h^{-1} \mathrm{Mpc}$, on the other hand, there are now large overdensities, Pegasus and Pisces, in the angular patch that previously contained the Hubble variance maximum, while on the opposite side of the sky in Hydra underdensities have emerged. These opposing influences will now even out the density gradients along the dipole axis. We can therefore understand why the dipole diminishes beyond $r \sim 60 h^{-1} \mathrm{Mpc}$.

As remarked above there is no significant LG frame dipole in shells 8 and 9 there is some evidence for such dipole in shell 10. Its direction, $(\ell, b)=\left(348^{\circ} \pm\right.$ $24^{\circ},-38^{\circ} \pm 14^{\circ}$ ) is at $\sim 83^{\circ}$, roughly orthogonal to the Shapley Concentration (SC), which is centred in shell 10 and extends into parts of shells 9 and 11 . Thus the small LG frame dipole in shell 10 (which points from an underdense region to Abell 576) is not correlated to the SC. Indeed in the region, $300^{\circ}<\ell<330^{\circ}, 15^{\circ}<b<45^{\circ}$, bounding the SC the numbers of positive and negative peculiar velocities with respect to the shell mean Hubble constant are equal in both shells 10 and 11, and almost equal in shell 9. The fact that the SC does not participate in a strong dipole may be due to significant mass concentrations on roughly the opposite side of the sky: see Ref. [54], Figs. 8, 9.

Since the quadrupole/dipole ratio is strong in shell 10, extra data is required to isolate the quadrupole before drawing strong conclusions about the magnitude of the dipole in this shell. However, we remark that effects on the Hubble flow at this scale might indeed be expected if the wall-to-wall distance-redshift is modified at the BAO scale: we are near one wall (defined by VirgoCentaurus-Hydra) which is separated from more distant structures such as the SC by the $100 h^{-1} \mathrm{Mpc} \mathrm{BAO}$ distance. Since the BAO enhancement is treated in the linear regime of perturbation theory, we might naturally

13 In the terminology of Ref. [10] the "Local Void North" comprises the region denoted "Delphinus" in Fig. 3 of Ref. [54] together with an adjacent large $\delta<0$ area extending to just above the galactic plane, $b \sim 6$, with $47^{\circ}<\ell<90^{\circ}$. The "Local Void South" of Ref. [10] is similarly much larger than the area marked "LV" in Fig. 3 of Ref. [54] and extends to adjacent $\delta<0$ regions above the galactic plane, with $\ell<47^{\circ}$. expect the magnitude of nearby Hubble flow variations driven by a BAO enhancement to be significantly smaller than the "nonlinear regime" dipole amplitude observed at $r \lesssim 55 h^{-1} \mathrm{Mpc}$. This would also suggest that much high quality data in the range $100 h^{-1} \lesssim r \lesssim 150 h^{-1} \mathrm{Mpc}$ is needed to fully constrain any potential variations.

\section{CORRELATION OF HUBBLE VARIANCE AND CMB DIPOLES}

Having demonstrated that Hubble flow is more uniform in the LG and LS frames as compared to the CMB frame, and that there is a strong dipole in these frames with an amplitude correlated to the residual monopole variations, the natural question to ask is: to what degree is the Hubble flow variance dipole correlated with the component of the CMB dipole that is usually attributed to the motion of the LG?

To answer this question we must compensate for our heliocentric motion with respect to the rest frame of the LG or LS by performing a boost to the relevant rest frame and examine the residual CMB temperature dipole in the rest frame in question. We create an artificial residual CMB dipole temperature map by subtracting a boosted CMB sky with temperature

$$
T^{\prime}=\frac{T_{0}}{\gamma\left(1-(v / c) \cos \theta^{\prime}\right)}
$$

from the corresponding observed pure temperature monopole plus dipole maps using the values of Ref. [2] assumed in Sec. II Here $v=v_{\mathrm{LG}}$ or $v=v_{\mathrm{LS}}$ as appropriate, and $\gamma \simeq 1$ since velocities are nonrelativistic. This leaves us with a residual temperature dipole with poles $\pm 5.77 \mathrm{mK}$ at $(\ell, b)=$ $\{(96.4,-29.3),(276.4,29.3)\}$ in the LG frame, and $\pm 5.73 \mathrm{mK}$ at $(\ell, b)=\{(90.3,-26.7),(270.3,26.7)\}$ in the LS frame. The dipole amplitudes have a $6.3 \%$ uncertainty arising principally from the uncertainty in the heliocentric velocity of the LG and LS frames. The LG residual temperature dipole is shown in Fig. [11.

We compute a correlation function directly by using HEALPIX to digitize both the residual temperature dipole map, and also the corresponding Hubble flow variance maps for the LG or LS frame as relevant. We quantify the correlation between the variance of that Hubble expansion and the residual CMB temperature dipole by the Pearson correlation coefficient

$$
\rho_{H T}=\frac{\sqrt{N_{p}} \sum_{\alpha} \bar{\sigma}_{\alpha}^{-2}\left(H_{\alpha}-\bar{H}\right)\left(T_{\alpha}-\bar{T}\right)}{\sqrt{\left[\sum_{\alpha} \bar{\sigma}_{\alpha}^{-2}\right]\left[\sum_{\alpha} \bar{\sigma}_{\alpha}^{-2}\left(H_{\alpha}-\bar{H}\right)^{2}\right]\left[\sum_{\alpha}\left(T_{\alpha}-\bar{T}\right)^{2}\right]}},
$$

where $T_{\alpha}$ is the temperature in the pixel with angular 


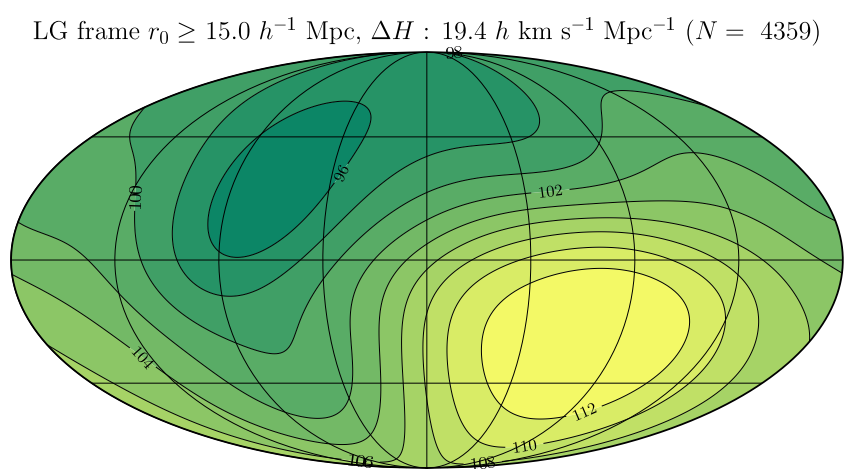

(a)

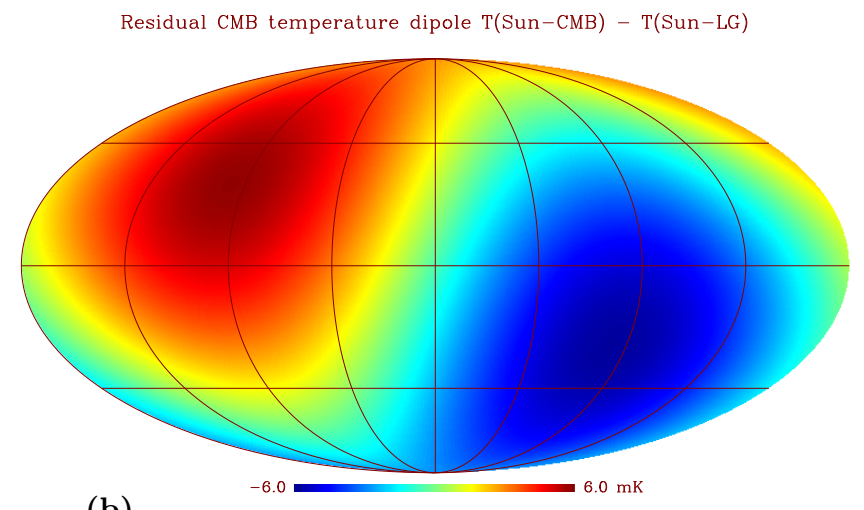

94

$H_{\alpha}\left(h \mathrm{~km} \mathrm{~s}^{-1} \mathrm{Mpc}^{-1}\right)$

(b)

FIG. 11: LG frame Hubble flow variance map for $r>15 h^{-1} \mathrm{Mpc}$ with IV weightings (panel(a)) compared to residual CMB temperature dipole in the LG rest frame (panel (b)). In all figures, the galactic longitudes $\ell=0^{\circ}, 180^{\circ}, 360^{\circ}$ are on the right edge, centre and left edge respectively.

coordinates $\alpha, \bar{T}$ is the mean temperature,

$$
\bar{H}=\frac{\sum_{\alpha}^{N_{p}} \bar{\sigma}_{\alpha}^{-2} H_{\alpha}}{\sum_{\alpha}^{N_{p}} \bar{\sigma}_{\alpha}^{-2}}
$$

$H_{\alpha}$ is given by (B5), $\bar{\sigma}_{\alpha}$ by (B8), and $N_{p}$ denotes the total number of pixels distributed over the sky. As we are considering a pure residual CMB temperature dipole there are no uncertainties in $T_{\alpha}$. Since HEALPIX partitions the celestial sphere into pixels of equal area, and since the CMB temperature dipole is assumed to be ideal, the only weighting in the sum comes from the measurement uncertainties of the Hubble flow.

With $\sigma_{\theta}=25^{\circ}$ we performed a correlation analysis between the Hubble variance dipole and the residual CMB temperature dipole in both the LG and LS frames, as the division radius, $r_{o}$, between the inner and outer spheres was varied. The results are shown in Fig. 12, We observe firstly that the correlation coefficient is negative since the maximum value of the Hubble parameter coincides with the minimum residual CMB temperature. The strongest anticorrelation is therefore represented by those values which are closest to -1 .

In all cases the result for the LS frame does not differ greatly from that of the LG frame. However, the anticorrelation is generally a bit stronger in the LG frame. The anticorrelation is stronger for the IV weighted sky maps in both frames. The anticorrelation is strongest in the outer sphere for $r_{o}=15 h^{-1} \mathrm{Mpc}$. As might be expected from Sec. IIIA, the anticorrelation remains strong in the outer sky maps for values of $r_{o}$ up to $40 h^{-1} \mathrm{Mpc}$. By contrast, the anticorrelation in the inner sphere is not at all strong for $r \leq r_{o}$ with small values of $r_{o}$. However, the anticorrelation in the inner sphere improves dramatically as $r_{o}$ is increased, and by the stage that we reach $r_{o}=50 h^{-1} \mathrm{Mpc}$ the anticorrelation is comparable in both spheres for the unweighted case, and stronger

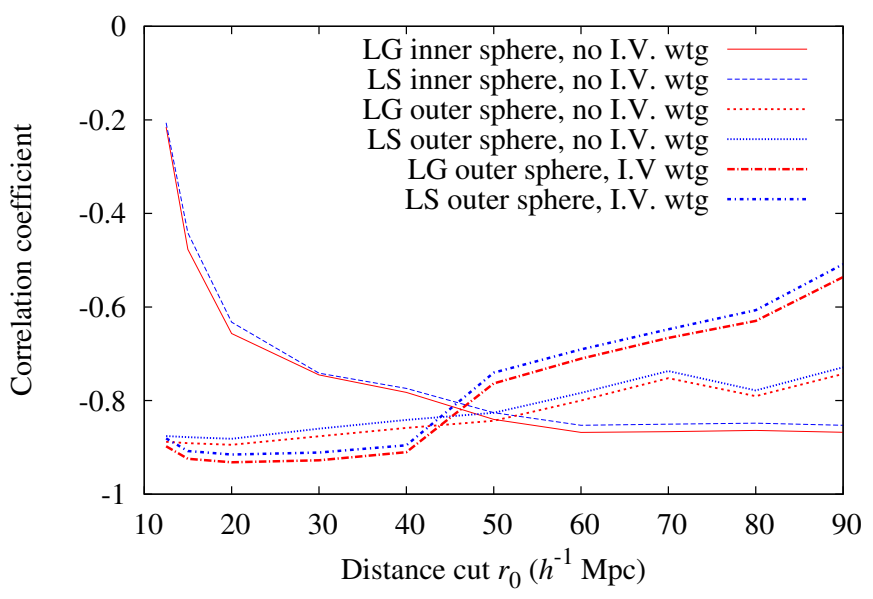

FIG. 12: Pearson correlation coefficient for the correlation of the residual CMB temperature dipole in the LG and LS rest frames, as compared to the Hubble variance sky map in inner $\left(r<r_{o}\right)$ and outer $\left(r>r_{o}\right)$ spheres, as $r_{o}$ is varied in the LG and LS rest frames. The correlation is computed both with and without the IV weighting.

in the inner sphere than in the outer sphere for the IV weighted case. There is no further improvement in the anticorrelation in the inner sphere for $r_{o}>60 h^{-1} \mathrm{Mpc}$, which is again consistent with the earlier indications that the structures responsible for the Hubble variance dipole are within $65 h^{-1} \mathrm{Mpc}$.

One final question is the extent to which the correlation depends on the Gaussian smoothing width, $\sigma_{\theta}$. We have checked this is two ways. Firstly, we have recomputed the correlation coefficient for a range of values of $\sigma_{\theta}$ for the $r>15 h^{-1} \mathrm{Mpc}$ map, the case which shows the strongest anticorrelation. The results are shown in Table III. We find that the anticorrelation in the IV weighted map is 


\begin{tabular}{lrrrr}
\hline$\sigma_{\theta}$ & LG u & LG w & LS u & LS w \\
$15^{\circ}$ & -0.8909 & -0.9056 & -0.8695 & -0.8750 \\
$20^{\circ}$ & -0.8945 & -0.9197 & -0.8774 & -0.8965 \\
$25^{\circ}$ & -0.8905 & -0.9240 & -0.8782 & -0.9077 \\
$30^{\circ}$ & -0.8847 & -0.9237 & -0.8769 & -0.9133 \\
$40^{\circ}$ & -0.8752 & -0.9187 & -0.8739 & -0.9160 \\
\hline
\end{tabular}

TABLE III: Correlation coefficient between the residual CMB temperature dipole sky map in the LG and LS rest frames, as compared to the Hubble flow variance sky map in the $r>15 h^{-1} \mathrm{Mpc}$ sphere, for different choices of Gaussian smoothing angle $\sigma_{\theta}$. Unweighted $(\mathrm{u})$ and IV weighted $(\mathrm{w})$ angular averaging is considered in each case.

stronger than the unweighted map for different choices of $\sigma_{\theta}$. Moreover, as well as the correlation coefficient in the LS frame being slightly weaker, it also varies a little more with smoothing angle. In the LG frame the correlation coefficient varies the least for varying $\sigma_{\theta}$ in the IV weighted case. Indeed to two significant figures the correlation coefficient of -0.92 is unchanged for $\sigma_{\theta}=$ $25^{\circ} \pm 5^{\circ}$.

A second check on the relation between the CMB temperature dipole and Hubble flow variance, that is completely independent of the Gaussian window averaging procedure, is given by evaluating a correlation coefficient between the residual temperature dipole and the raw COMPOSITE peculiar velocity data in the LG frame. In this case the relevant correlation coefficient is given by

$$
\begin{aligned}
& \rho_{v T}= \\
& \frac{\sqrt{N} \sum_{i} \sigma_{i}^{-2}\left(v_{i}-\bar{v}\right)\left(T_{i}-\bar{T}\right)}{\sqrt{\left[\sum_{i} \sigma_{i}^{-2}\right]\left[\sum_{i} \sigma_{i}^{-2}\left(v_{i}-\bar{v}\right)^{2}\right]\left[\sum_{i}\left(T_{i}-\bar{T}\right)^{2}\right]}} .
\end{aligned}
$$

where $v_{i}$ denote the peculiar velocities and $N$ is the number of data points. The weighted average peculiar velocity should approach zero for a large number of data points: here $\bar{v}=-64.9 \mathrm{~km} \mathrm{~s}^{-1}$ with a standard deviation of $722.4 \mathrm{~km} \mathrm{~s}^{-1}$. For the COMPOSITE LG-frame velocities from the data with $r \geq 15 h^{-1} \mathrm{Mpc}, N=4359$ and we obtain $\rho_{v T}=-0.35$. The magnitude of this correlation is naturally lower than it is for the weighted grid data which we calculated above due to the scatter in these data, but the well defined number of points implies we have better statistical tools to quantify our confidence that the correlation is indeed nonzero. We test this by evaluating the variable

$$
t=\rho_{v T} \frac{\sqrt{\nu}}{\sqrt{1-\rho_{v T}^{2}}},
$$

where $\nu=N-2$ is the number of degrees of freedom. If there is no correlation, the test variable $t$ in (15) should follow the standard normal distribution $N(0,1)$. For $\nu=$ 4357 and $\rho_{v T}=-0.346$, we obtain the value $t=-24.35$, i.e., a deviation of more than $24 \sigma$. This is extremely strong statistical evidence for a nonzero (anti)correlation.

We therefore have strong evidence that the dipole features of the two maps in Fig. 11 are related. From Fig. 13 we see that in the IV weighted sky map the cooler residual CMB temperature pole (marked by a cross) lies just $7.4^{\circ}$ from the maximum of the Hubble flow variance, well within the $1 \sigma$ contour. The hotter residual CMB temperature pole is separated by $22^{\circ}$ from the minimum of the Hubble flow variance, however, and lies $10^{\circ}$ outside the $1 \sigma$ contour but is within $3 \sigma$. It remains to be seen whether the uncertainty estimates in this case are significantly affected by the choice of weighting scheme. In particular, the left hand panel of Fig. 1 of FWH10 shows that with a maximum likelihood estimate based on traditional IV weightings there are a substantial number of very strongly weighted data points in the COMPOSITE sample in the region which coincides with that of minimum $\bar{\sigma}_{\alpha}$ to the north of residual CMB temperature pole in Fig. 13(b). With the alternative minimum variance weightings shown in the right hand panel of Fig. 1 of FWH10 the same data points are not strongly weighted.

\section{ORIGIN OF THE CMB DIPOLE}

Ever since the first bounds were placed on the anisotropy of the CMB in the mid 1960s [55] it has been assumed that the dipole anisotropy represents a measurement of our motion with respect to our surface of average homogeneity [56, 57]. Our results clearly show, however, that for spherical (monopole) averages the Hubble flow is closer to being uniform in the frame of the LG or LS, rather than the frame indicated by treating the $\mathrm{CMB}$ dipole as being entirely due to a boost. This is completely unexpected in the standard framework, since the cosmic rest frame and the frame of minimum Hubble flow variance should be one and the same. Moreover, we find a dipole in the LG frame whose amplitude diminishes in correlation to the diminishing of the monopole variations. This dipole is strongly correlated with the residual CMB temperature dipole.

\section{A. Puzzles of the bulk flow formalism}

If we set aside the question of the monopole variation and its relative uniformity in different rest frames, then a dipole is of course expected in the standard peculiar velocity framework. Indeed, in the standard framework the dipole pattern we observe was detected by Jha, Riess and Kirshner [60] in a sample of 69 MLCS2k2reduced SneIa distances in the range $0.005 \leq z \leq 0.025$, and interpreted by them as a positive detection of the motion of the LG with magnitude $541 \pm 75 \mathrm{~km} \mathrm{~s}^{-1}$ towards $(\ell, b)=\left(258^{\circ} \pm 18^{\circ}, 51^{\circ} \pm 12^{\circ}\right)$, which is $2 \sigma$ con- 

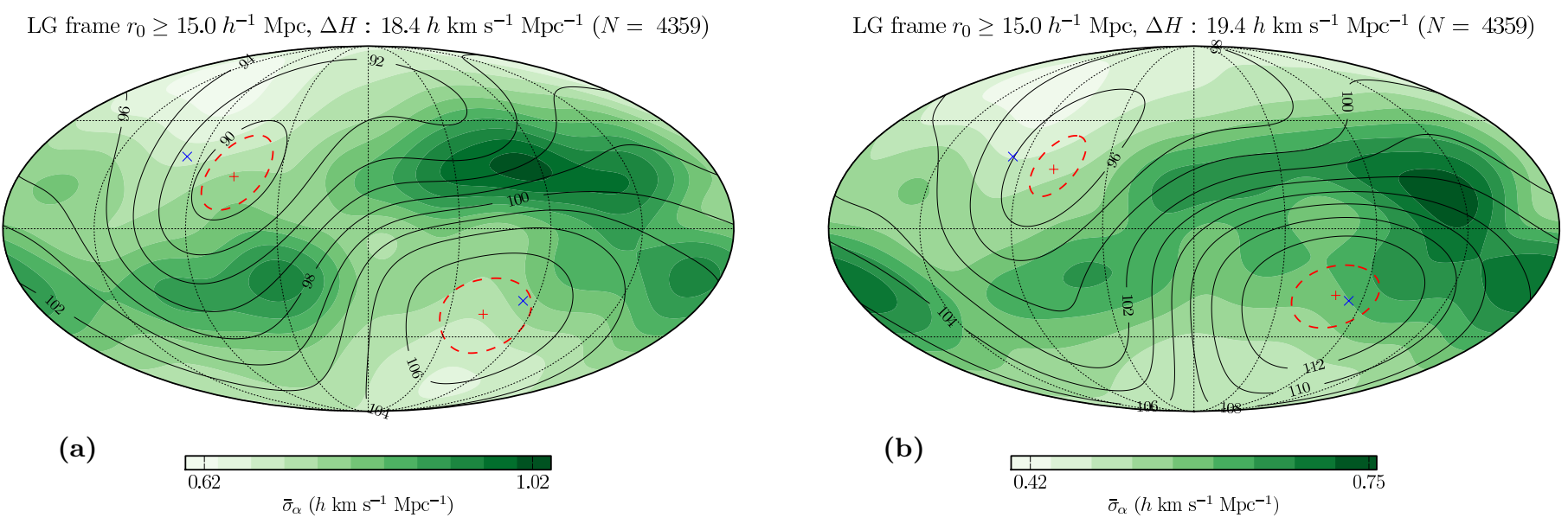

FIG. 13: Angular variance of the LG rest frame Hubble flow given by $H_{\alpha}$ in the $r>15 h^{-1}$ Mpc range (solid lines), overlaid with angular uncertainties $\bar{\sigma}_{\alpha}$ (colour map contours): (a) with no IV weights; (b) with IV weights (B9). The red dashed circle indicates a $1 \sigma$ region around the maximum/minimum. Blue crosses indicate the residual CMB temperature dipole poles. In all figures, the galactic longitudes $\ell=0^{\circ}, 180^{\circ}, 360^{\circ}$ are on the right edge, centre and left edge respectively.

sistent with the amplitude and magnitude of the boost of the LG with respect to the CMB frame. The problem was re-examined by Gordon, Land and Slosar 61. using a sample of 61 SALT-reduced SneIa in the range $0.0076 \leq z \leq 0.124$, who found $v_{\mathrm{LG}}=697 \pm 137$ in the direction $(\ell, b)=\left(220^{\circ} \pm 14^{\circ}, 32^{\circ} \pm 11^{\circ}\right)$. When the linear theory was corrected to account for correlated peculiar velocities, the uncertainties on these values were increased giving $v_{\mathrm{LG}}=690 \pm 201 \mathrm{~km} \mathrm{~s}^{-1} \mathrm{Mpc}^{-1}$ towards $(\ell, b)=\left(257^{\circ} \pm 24^{\circ}, 29^{\circ} \pm 16^{\circ}\right)$ [61], which is $1 \sigma$ consistent with the LG boost with respect to the CMB frame.

Phenomenologically, the above results in the linear theory show agreement with the expected LG boost, and the SneIa sample of Ref. [60] appears to show convergence to the expected result within the same radial scale we find in the COMPOSITE sample: in Sec. [I] we saw that the LG frame is within $1.36 \sigma$ of uniform in spherical shells with $\bar{r}_{s} \geq 69 h^{-1} \mathrm{Mpc}$, and in Sec. IIIB we saw that the large dipole feature in the LG frame diminished to its residual value by $r_{o}=55 h^{-1} \mathrm{Mpc}$. Thus the structures responsible for both the monopole and the dipole variation in the Hubble flow appear to be foregrounds within roughly ${ }^{14} 65 h^{-1} \mathrm{Mpc}$, or $z \simeq 0.022$.

In the standard peculiar velocity framework, however, demonstrating the convergence of bulk flows on this scale has proved challenging. In this framework peculiar velocities are estimated from linearly perturbed FLRW model

14 There is some ambiguity in defining this transition scale. Conservatively, we take the scale to be the average of the mean distances of shells $5^{\prime}$ and 6 , where $\delta H_{s}$ first drops below $1.5 \sigma$ difference from uniform. according to 62

$$
\mathbf{v}(\mathbf{r})=\frac{H_{0} \Omega_{M 0}^{0.55}}{4 \pi} \int \mathrm{d}^{3} \mathbf{r}^{\prime} \delta_{m}\left(\mathbf{r}^{\prime}\right) \frac{\left(\mathbf{r}^{\prime}-\mathbf{r}\right)}{\left|\mathbf{r}^{\prime}-\mathbf{r}\right|^{3}}
$$

where $\delta_{m}(\mathbf{r})=(\rho-\bar{\rho}) / \bar{\rho}$ is the density contrast, and the power 0.55 of the matter density parameter $\Omega_{M 0}$, rather than 0.6 , gives improved accuracy for models with dark energy [63].

The search for convergence of bulk flows within this framework has a three decade history, which is summarized by Lavaux et al. [12] and by Bilicki et al. 64]. Contrary to earlier investigations [54] Lavaux et al. [12] failed to find convergence in the 2MASS survey on scales up to $120 h^{-1} \mathrm{Mpc}$ : less than half the amplitude was generated on scales $40 h^{-1} \mathrm{Mpc}$, and whereas most of the amplitude was generated within $120 h^{-1} \mathrm{Mpc}$ the direction did not agree. Bilicki et al. [64] analysed a larger sample in the 2MASS survey using a different methodology and failed to find convergence within $150 h^{-1} \mathrm{Mpc}$.

In the COMPOSITE sample Watkins, Feldman and Hudson [18] failed to see convergence of peculiar velocities to the $\mathrm{CMB}$ dipole on scales of $50 h^{-1} \mathrm{Mpc}$. Even more puzzling in their results is the suggestion that the bulk flow actually increases with increasing scale above $20 h^{-1} \mathrm{Mpc}$ (see their Fig. 5). In the peculiar velocity framework, the only way to understand how a larger volume can have a larger bulk flow than a smaller volume contained within it is to posit that the inner volume has an additional compensating motion in the opposite direction. While possible, this arrangement seems unexpected at best. Furthermore, it offers no explanation for the surprising degree of uniformity of the spherically averaged Hubble law in the LG frame found in Sec. II. 


\section{B. Differential expansion of space as a foreground anisotropy}

The problems and puzzles of the standard linear theory alone might not justify a radical revision of the standard formalism. However, our new results concerning the monopole Hubble flow variations, their radial correlation with change of amplitude of the dipole variations, and the relative uniformity of the Hubble flow in the two frames, all defy explanation in the standard framework. Theoretically therefore the natural course of action is to go back to first principles.

Another potential source of the dipole anisotropy has been previously considered [58], namely the Rees-Sciama effect [59] (the nonlinear version of the integrated SachsWolfe effect). However, to generate a dipole anisotropy of magnitude $\delta T / T \sim 10^{-3}$ in this fashion would require a void of radius $300 h^{-1} \mathrm{Mpc}$ - an order of magnitude larger than than that of the observed voids - and furthermore it would also induce a quadrupole of the same magnitude as the dipole [58]. This effect therefore fails as a potential explanation.

The Rees-Sciama effect and other late-epoch sources of CMB anisotropies such as the Sunyaev-Zel'dovich effect are conventionally considered as the effects of inhomogeneities embedded in a background FLRW cosmology that applies at all scales. However, from first principles, differences in the observed mean CMB temperature will be also generated if the universe has grown sufficiently inhomogeneous by the present epoch that the distance to the surface of photon decoupling is slightly different in different directions. Over large distances photon paths see an average of all the structures, but the last section of a photon journey below the scale of statistical homogeneity will be influenced by the peculiar foregrounds.

Our universe is in fact sufficiently inhomogeneous on scales $\lesssim 100 h^{-1} \mathrm{Mpc}$ that the differential expansion of void and wall regions can be expected to produce differences in the distance to the surface of last scattering, if we are abandon the assumption that an exact FLRW description must apply at all scales. Our proposal is potentially consistent with a variety of non-FLRW models, such as the timescape scenario [34, 35], an alternative to the standard cosmology which has remained viable in tests to date [37, 65 68]. We do not wish to focus on any particular model-dependent estimates here, but rather to point out that the global FLRW geometry is a very special one which encourages conceptual simplifications that are not demanded by full general relativity. We will determine the magnitude of the effect required to produce the observed residual temperature dipole using the $\Lambda$ CDM model phenomenologically for distance estimates.

As long as the average evolution of the universe can be described by an average cosmic scale factor ${ }^{15}$ which is

${ }^{15}$ It is not necessary for the average evolution to obey the Fried- related to the observed cosmological redshift by $a_{0} / a=$ $1+z$, and which is inversely proportional to the mean CMB temperature, $T \propto 1 / a$, then a small change, $\delta z$, in the redshift of the surface of photon decoupling - due to foreground structures - will induce a CMB temperature increment $T=T_{0}+\delta T$, where $T_{0}=2.725 \mathrm{~K}$ is the present epoch mean temperature and

$$
\frac{\delta T}{T_{0}}=\frac{-\delta z}{1+z_{\mathrm{dec}}}
$$

$z_{\mathrm{dec}}=1089$ being the mean redshift of photon decoupling. For the LG residual dipole the increment $\delta T= \pm(5.77 \pm 0.36) \mathrm{mK}$ represents a redshift increment $\delta z=\mp(2.31 \pm 0.15)$.

The comoving distance of the surface of photon decoupling is given by

$$
D=\frac{c}{H_{0}} \int_{1}^{1+z_{\mathrm{dec}}} \frac{\mathrm{d} x}{\sqrt{\Omega_{\Lambda 0}+\Omega_{M 0} x^{3}+\Omega_{R 0} x^{4}}}
$$

in the standard spatially flat $\Lambda$ CDM model, where $\Omega_{\Lambda 0}=$ $1-\Omega_{M 0}-\Omega_{R 0}$ and $\Omega_{R 0}=4.15 h^{-2} \times 10^{-5}$. If we take $\Omega_{M 0}=0.25$ and $h=0.72$ we find that the comoving distance increment of $\delta D=\mp(0.33 \pm 0.02) h^{-1} \mathrm{Mpc}$ is what is required to generate the residual CMB dipole in the $\mathrm{LG}$ frame. For $\Omega_{M 0}=0.30$ the value is slightly reduced to $\delta D=\mp(0.32 \pm 0.02) h^{-1} \mathrm{Mpc}$. For the timescape model [37] the value is similar, with possible small differences depending on parameter values.

The results of the previous sections suggest that the structures responsible for the Hubble flow variance dipole lie at most within $65 h^{-1} \mathrm{Mpc}$. Since the differences in the distance to the surface of last scattering occur effectively at $z=0$, a $0.35 h^{-1} \mathrm{Mpc}$ difference in distance therefore would amount to a maximum $0.5 \%$ difference on these scales. Even if the whole difference was taken up within an average distance scale of $30 h^{-1} \mathrm{Mpc}$, leading to a $1 \%$ effect, this is still within the regime of plausibility given the degree of Hubble flow variance we observe in the COMPOSITE sample.

Our picture then, as in Fig. 1 is one of differential expansion of void and wall regions at late epochs leading to distance differences of up to the order of $1 \%$ between walls and voids on $30 h^{-1}-70 h^{-1} \mathrm{Mpc}$ average distance scales, a scale determined by the size of the largest typical nonlinear structures [38, 39] and their random correlations. While such differences are not isolated to our own immediate vicinity, when light travels over scales larger than the scale of statistical homogeneity the differences generally average out on any typical line of sight. It is on the last stretch of the journey, when the typical

mann equation for this statement to be true. In particular, it also applies to the timescape cosmology [34, 35, 37] which describes the average evolution of an ensemble of void and wall regions in the Buchert averaging scheme [69, 70], with additional physical assumptions about the interpretation of physical observables. 
nonlinear structures subtend a large angle on the sky, that the particular foreground inhomogeneities peculiar to our own location give a strong net anisotropic contribution $^{16}$. In our case the largest foreground density gradient defines an axis with the void direction yielding a slightly larger distance than average and a net CMB temperature decrement, and the opposite wall direction a slightly smaller distance than average and a CMB temperature increment.

Our proposal for an alternative origin for part of the CMB dipole should only have a very significant effect on the angular power spectrum on scales larger than $15^{\circ}$, on which anomalies are seen. High precision measurements of the acoustic peaks [71, 72] on scales of less than $1^{\circ}$ will be influenced in subtle ways, as discussed in Sec. VIG below.

\section{Ray-tracing estimates of dipole and quadrupole strengths}

One important question remains: why is the CMB dipole so large compared to other multipoles if a substantial contribution is due to a foreground anisotropy in the distance-redshift relation? In the case of the ReesSciama effect, for example, the quadrupole is comparable to the dipole 58]. The Rees-Sciama effect for structures on scales $\lesssim 100 h^{-1} \mathrm{Mpc}$ is estimated to be $\delta T / T \sim 10^{-7}$ $10^{-6}[73,[74]$, and is a secondary effect compared to the one we propose. Nonetheless, the relative strength of the observed CMB multipoles must be understood if our proposal is to be a viable explanation for the unexpected results concerning the uniformity of the Hubble flow.

The problem of describing the propagation of light through a realistic inhomogeneous structure is a complicated one. However, the plausibility of our proposal is readily demonstrated by adapting results [75] found in previous studies of ray tracing of the CMB sky as seen by an off-centre observer in a Lemaître-TolmanBondi (LTB) void. Although past investigations focused on large voids as toy models for explaining luminosity distances of supernovae without dark energy [75, 76], the ray-tracing results can also be applied to voids of any scale, and in particular to those of the size of observed local structures [54].

Alnes and Amarzguioui [75] showed that a Newtonian

16 The BAO scale is close to the statistical homogeneity scale, near $\sim 110 h^{-1} \mathrm{Mpc}$, and therefore we may also expect a small anisotropic enhancement to the Hubble flow near the BAO scale. Since this is a linear perturbation the amplitude of the anisotropy is likely to be smaller. Furthermore, once one considers larger shells then the angles subtended by typical nonlinear structures are smaller relative to the centre (see Fig. 1), so multipoles higher than the dipole are likely to come into play. approximation, with an effective peculiar velocity

$$
\frac{v_{\mathrm{p}}}{c}=\frac{\left(h_{\mathrm{in}}-h_{\mathrm{out}}\right) d_{\mathrm{off}}}{2998 \mathrm{Mpc}}
$$

where $H_{\text {in } 0}=100 h_{\text {in }} \mathrm{kms}^{-1} \mathrm{Mpc}^{-1}$ and $H_{\text {out } 0}=$ $100 h_{\text {out }} \mathrm{km} \mathrm{s}^{-1} \mathrm{Mpc}^{-1}$ are the Hubble constants inside and outside the void, and $d_{\text {off }}$ is the distance of the observer from the centre in Mpc, yields results for CMB multipoles which are numerically close to the ray-traced result. In this approximation the ratio of the quadrupole coefficient, $a_{20}$, to dipole coefficient, $a_{10}$, is

$$
\frac{a_{20}}{a_{10}}=\sqrt{\frac{4}{15}} \frac{\left(h_{\mathrm{in}}-h_{\mathrm{out}}\right) d_{\mathrm{off}}}{2998 \mathrm{Mpc}} .
$$

Our position in the thin filamentary Local Sheet on the edge of the Local Void [10] complex, at a distance $\gtrsim 12 h^{-1} \mathrm{Mpc}$ from the nearest wall region (Virgo Cluster), means that our location is sufficiently void-like for the approximation (19), (20) to be reasonable.

A value of $H_{\text {in } 0}-H_{\text {out } 0}$ can be estimated from the magnitude of the dipole in the LG frame from the first 4 shells in Table VII, which correspond to the range over which the LG frame dipole is strong before rapidly decreasing. The weighted mean is $\beta=16.1 h \mathrm{~km} \mathrm{~s}^{-1} \mathrm{Mpc}^{-1}$ in the unprimed shells $1-4$, and $\beta=14.1 h \mathrm{~km} \mathrm{~s}^{-1} \mathrm{Mpc}^{-1}$ in the primed shells 1 '-4', so that $H_{\text {in } 0}-H_{\text {out } 0} \simeq \beta=$ $(15.1 \pm 1.0) h \mathrm{~km} \mathrm{~s}^{-1} \mathrm{Mpc}^{-1}$. To reproduce the effective peculiar velocity $v_{\mathrm{p}}=635 \pm 38 \mathrm{~km} \mathrm{~s}^{-1}$ inferred for Local Group ${ }^{17}$ [10], eq. (19) therefore requires us to be $d_{\mathrm{off}}=(42 \pm 3) h^{-1} \mathrm{Mpc}$ from the centre of a void. As seen in Fig. 9 this does indeed match to the scale at which the dipole magnitude $\beta$ decreases rapidly in the LG frame, demonstrating the consistency of the approximation (19).

Using (19), (20) we find that for the values of $\beta$ and $v_{p}$ we find $a_{20} / a_{10}=0.0011 \pm 0.0002$. A quadrupole/dipole ratio $\sim 0.1 \%$ for relative $\mathrm{CMB}$ anisotropies is certainly within observational bounds. In fact, the Newtonian approximation (20) is known to overestimate the quadrupole by a factor of 2 [75]. This is confirmed in explicit ray tracing simulations in an LTB model with structures of the scales above. In particular, using the parametrization of Ref. 77] (model 1), with parameters adjusted so that $\beta=15.1 \mathrm{hm} \mathrm{s}^{-1} \mathrm{Mpc}^{-1}$ and $d_{\text {off }}=$ $42 h^{-1} \mathrm{Mpc}$ within a void of radius $54 h^{-1} \mathrm{Mpc}$ embedded in a background FLRW model with $\Omega_{M 0}=0.3=1-\Omega_{\Lambda 0}$, one finds $a_{20} / a_{10} \approx 0.0005[78]$.

The actual matter distribution is of course more complicated than that of a single LTB void in a homogeneous background, and the problem of determining the average propagation of CMB photons through the foregrounds is closely related to how to realistically average foreground

\footnotetext{
17 Numerical values in Ref. 75] differ by roughly a factor two, as they neglected to subtract the contributions of the known motions of the sun and galaxy to the CMB dipole.
} 
density fields. Work is in progress to trace rays through exact solutions of Einstein's equations which more closely emulate our peculiar density foregrounds [79]. The nonsymmetric Szekeres models [80] enable one to model the density gradient provided by a void and adjacent cluster, with the parametrization of Sec. IIIC of Ref. [81]. For example, by placing an observer $12 h^{-1} \mathrm{Mpc}$ from the centre of a $27 h^{-1} \mathrm{Mpc}$ radius void and at a distance of $15 h^{-1} \mathrm{Mpc}$ from an overdensity, one finds $a_{20} / a_{10} \approx 0.01$ [78], which is larger than in the LTB case but still observationally acceptable. (Reducing this configuration to spherical symmetry [82, 83], decreases the ratio to 0.0001 , which is within a factor of 2 agreement with Eq. (20).) More detailed results of this ray-tracing analysis will be presented in a forthcoming article [79].

\section{DISCUSSION}

In summary, we have shown with decisive Bayesian evidence that when averaged in spherical shells on scales $\lesssim 150 h^{-1} \mathrm{Mpc}$ the Hubble flow is more uniform in the rest frame of the LG or LS than in the standard "rest frame" of the CMB. An exception occurs for shells in the range $40 h^{-1} \lesssim r \lesssim 60 h^{-1} \mathrm{Mpc}$, where the boost to the CMB frame "almost works". This is echoed in the dipole variation: the CMB frame dipole in shell 4 at mean distance $\bar{r}_{4}=44.5 h^{-1} \mathrm{Mpc}$ is not statistically significant, but subsequently increases at larger radial distances. By contrast the LG frame dipole diminishes greatly, becoming consistent with zero in shells 8 and 9. A significant CMB frame dipole remains in all outer shells, its direction being consistent with previous peculiar velocity studies. While a smaller magnitude LG frame dipole reappears in shell 10 in the range $110 h^{-1} \lesssim r \lesssim 150 h^{-1} \mathrm{Mpc}$, its direction is different to the inner dipole, and unlike the inner dipole is not correlated with the residual CMB temperature dipole in the LG frame.

These results are difficult to reconcile with the standard kinematic interpretation of the Local Group moving in response to the gravitational attraction of the clustering dipole. However, they are consistent with a foreground different expansion of space of order $0.5 \%$ due to the density gradients of nonlinear structures on scales $30 h^{-1} \lesssim r \lesssim 62 h^{-1} \mathrm{Mpc}$, which we have tentatively identified in Sec. IIID, Such a foreground would also affect measurements on large scales $\gg 100 h^{-1} \mathrm{Mpc}$ as the typical distance for a given redshift would vary from the average distance by up to $0.35 h^{-1} \mathrm{Mpc}$, with a roughly dipolar distribution on the sky.

This suggestion challenges a basic assumption of observational cosmology, and if upheld by future investigations, will clearly have important consequences. From general relativity there is no a priori reason for assuming that space is flat with a simple Doppler law expansion on scales $\lesssim 150 h^{-1} \mathrm{Mpc}$. Nonetheless this assumption is so firmly embedded in much of the practice of observational cosmology that it is nontrivial to disentangle the conse- quences of revisiting this assumption. Here we will sketch just some of the directions which should be pursued in more detailed investigations.

\section{A. From bulk flows to Hubble flow variance}

A differential expansion of space arising from the differing histories of regions of different density may simply lead to an alternative interpretation of many of the phenomenological results of the peculiar velocity framework. For example, redshift space distortions are well understood in terms of the Kaiser effect [84]. Before tackling the broader treatment of redshift space distortions, we need to begin by understanding how convergence of the nearby Hubble flow to the CMB dipole should be defined.

The fractional dipole anisotropy, typically of order $\beta / H_{d} \sim 9 \%$ for shells in the range $30 h^{-1} \lesssim \bar{r}_{s} \lesssim 62 h^{-1} \mathrm{Mpc}$ (c.f. Fig. 9), is much larger than the $0.5 \%$ differential expansion required on these scales to produce the $\mathrm{CMB}$ dipole. However, these values of $\beta / H_{d}$ also include the monopole variation (c.f. Fig. 3), which first needs to be factored out before additional angular variation is considered.

A starting point for a multipole expansion of the Hubble flow variation would be to define the average comoving distance, $D$, to a redshift, $z<z_{\text {hom }}$, within the scale of statistical homogeneity, $z_{\text {hom }}$, according to

$$
D(z)=c \int_{0}^{z} \frac{\mathrm{d} z_{s}}{H_{s}\left(z_{s}\right)} .
$$

Here $H_{s}\left(z_{s}\right)$ is computed exactly as in (2) except that the shells on which the linear regression is performed are chosen by redshift ranges, $z_{s}<z \leq z_{s}+\sigma_{z}$, where $\sigma_{z}$ represents the width of the radial shells in redshift. For example, in Sec. III we chose shells of radial width $12.5 h^{-1} \mathrm{Mpc}$, which corresponds to taking $c \sigma_{z}=1250 \mathrm{~km} \mathrm{~s}^{-1}$, or $\sigma_{z}=0.0042$. The average luminosity and angular diameter distances are related to $D(z)$ by $D_{L}=(1+z) D=(1+z)^{2} D_{A}$.

The radial shell width, $\sigma_{z}$, is analogous to the angular smoothing width, $\sigma_{\theta}$, of Sec. IIIA The minimum shell width possible is set by the largest bound structures that exist, since a regression (2) can only be calculated on scales over which space is expanding and a Hubble law is defined. Thus $c \sigma_{z} / H_{0}$ should be larger than the diameter of the largest rich clusters of galaxies, which justifies the choice made in Sec. III There will be similar restrictions on the choice of smoothing angle, $\sigma_{\theta}$, depending on the details of angular averaging procedure.

For each shell redshift, $z_{s}$, the angular corrections $H\left(z_{s}, \theta, \phi\right)-H\left(z_{s}\right)$ will lead to corrections to the mean comoving distance (21) which might be expanded as multipoles. Eq. (21) defines the monopole contribution to the distance-redshift relation. Convergence of the Hubble flow variance in a large data set would then be obtained if the dipole anisotropy converges to a fixed 
value for $z>z_{\text {conv }}$, where our preliminary investigations suggest that the convergence scale is at least of order $z_{\text {conv }} \simeq 0.022$. To consistently account for the residual CMB temperature dipole the residual anisotropy in $D(z)$ would be up to the order of $\pm 0.35 h^{-1} \mathrm{Mpc}$, with the exact value depending on the cosmological model.

A further question to be resolved by future surveys is the split between the linear and nonlinear regimes in cosmology. In particular, the BAO enhancement is assumed to be in the linear regime of perturbation theory about a FLRW model in the standard model. Our results are tentatively consistent with the possibility that convergence of Hubble flow variance by $z_{\text {conv }} \simeq 0.022$, apart from very small features at $r_{\text {bao }} \pm r_{\text {off }}$, where $r_{\text {bao }}$ is the effective comoving BAO scale and $r_{\text {off }}$ is the distance by which we are offset from the centre of the nearest (VirgoCentaurus-Hydra) wall. Much more data is required in the range $100 h^{-1} \lesssim r \lesssim 150 h^{-1} \mathrm{Mpc}$ to confirm this. If confirmed, it would be consistent with the notion that scales $z \lesssim z_{\text {conv }}$ are in the "nonlinear regime" while the BAO scale is in the "linear regime" 18.

\section{B. The minimum Hubble variance rest frame}

The multipole expansion of the Hubble flow variance proposed in Sec. VIA should ideally be performed in the rest frame in which the radial variance in the Hubble flow with respect to the asymptotic global average $\bar{H}_{0}$ is minimized. We have shown that this frame is closer to the $\mathrm{LG}$ rest frame than the CMB rest frame. The flow in LS frame is very close to that of the LG frame, but very slightly more variable.

We should also consider the possibility that the LG has an additional peculiar velocity with respect to the frame in which variance in the Hubble flow is minimized. Such a task is feasible, even if computationally intensive, and we will report on this in future work. A further comparison is to independently determine the frame with the greatest anticorrelation between the residual CMB temperature dipole and the Hubble flow variance dipole. Does such a frame agree with the minimum Hubble variance frame, within uncertainties?

\section{The Hubble bubble and type Ia supernova systematics}

Type Ia supernovae (SneIa) provide the standardizable candles which are the cornerstone of many current cosmological tests. The use of SneIa is currently limited by sys-

18 Such an interpretation just relies on there existing a scale of statistical homogeneity above which average cosmological evolution can be described. It is not necessary for the average evolution to be exactly that of a homogeneous isotropic FLRW model. tematic uncertainties, and differences in cosmological parameter estimations can be obtained when different light curve reduction methods are used. In the SALT/SALTII method [85, 86] empirical light curve parameters are marginalized together with cosmological parameters over the whole data set, whereas in the MLCS2k2 method [60] template light curves are determined by minimizing the distance modulus residuals of a training set of nearby SneIa, which lie within the range in which the Hubble flow is linear, yet are sufficiently distant for peculiar velocities to be negligible compared to the Hubble-flow $c z$.

If the cosmic rest frame is taken to be that of minimum Hubble flow variance on $\lesssim 100 h^{-1} \mathrm{Mpc}$ scales, and if such a frame is close to the LG frame, then an interesting systematic issue arises. In both light curve calibration methods one seeks to minimize the distance modulus residuals with respect to a nearby global linear Hubble law, and by convention such a minimization in the rest frame of the CMB rather than the LG frame. The Union [87], Constitution [88] and Union2 samples [89] contain a significant number of data points in the range ${ }^{19}$ $0.015 \lesssim z \lesssim 0.02$ which is below the scale $z_{\text {conv }}$ but is still conventionally deemed to be "within the Hubble flow".

Interestingly, the redshift range $0.012 \lesssim z \lesssim 0.02$ corresponds to the range $36 h^{-1} \lesssim r \lesssim 60 h^{-1} \mathrm{Mpc}$ over which the boost to the CMB rest frame was found to produce a smaller deviation from a uniform Hubble flow than in the LG frame (c.f. Fig. 3), even though the Hubble flow is significantly more uniform in the LG frame overall. Thus the fact that the boost to the CMB rest frame appears to best compensate for structures in the range $30 h^{-1} \lesssim r \lesssim 62 h^{-1} \mathrm{Mpc}$ may have led to a misidentification of the minimum redshift, $z_{\text {conv }}$, at which a single global linear Hubble law can be safely assumed.

Fig. 3 and Table 1 indicate that in the LG frame convergence to an almost uniform Hubble flow is achieved by $\bar{r} \simeq 65 h^{-1} \mathrm{Mpc}$ or $z_{\mathrm{conv}} \simeq 0.022$. This scale coincides roughly with the cutoff scale of the Hubble bubble identified in the supernovae data by Zehavi et al. 91] at $z=0.24$, and confirmed by Jha, Riess and Kirshner [60], using a MLCS2k2 sample with a reddening parameter $R_{V}=3.1$. We note that over the range $60 h^{-1} \lesssim r \lesssim 70 h^{-1} \mathrm{Mpc}$ the Hubble flow is somewhat closer to uniform in the LG frame as opposed to the CMB frame, and the variance in these shells in either frame is less than the $6.5 \pm 2.2 \%$ found in Ref. 91]. However, Refs. [60, 91] worked with a far simpler model of Hubble flow variance in which the sample was divided into inner and outer spheres.

We have checked the result of Zehavi et al. [91] by

19 By contrast Kessler et al. [90], for their full MLCS2k2 Nearby+SDSS+SNLS+ESSENCE+HST sample, took a minimum redshift of $z=0.0218$. There are differences in cosmological parameters estimated from the SDSS sample [90] and the Union, Constitution and Union2 samples. 
repeating their analysis on the COMPOSITE sample, fitting a simple linear Hubble law to the 2222 data points in the interval $30 h^{-1}<r \leq 70 h^{-1} \mathrm{Mpc}$ chosen for the inner shell in Ref. [91]. We find $H_{0}=$ $(104.5 \pm 0.6) h \mathrm{kms}^{-1} \mathrm{Mpc}^{-1}$ in the CMB frame, and $H_{0}=(105.1 \pm 0.6) h \mathrm{~km} \mathrm{~s}^{-1} \mathrm{Mpc}^{-1}$ in the LG frame. These values are respectively $4.40 \pm 0.08 \%$ and $4.06 \pm$ $0.07 \%$ larger than the global asymptotic values of $\bar{H}_{0}$ determined in Sec. III. They are somewhat lower but consistent with the $6.5 \pm 2.2 \%$ effect found in Ref. 91.

The $30 h^{-1}<r \leq 70 h^{-1} \mathrm{Mpc}$ range chosen in Ref. 91] is equally divided between regimes in which the LG frame Hubble flow is closer to uniform in the COMPOSITE data, and alternatively in which the CMB frame is closer to uniform, as seen in Fig. 3. This explains why the average values of $H_{0}$ in this range are closer to each other than those determined in Sec. II by fitting a simple linear Hubble law to the whole sample. The latter values, which amounted to $8.8 \pm 0.2 \%$ in the $\mathrm{CMB}$ frame and $3.37 \pm$ $0.07 \%$ in the LG frame, might be taken as a sharper estimate of the Hubble bubble effect.

The existence of a Hubble bubble has been controversial since as far as SneIa data analysis is concerned the presence of the effect is dependent on the details of the treatment of extinction and reddening by dust [92]. A Hubble bubble is found if dust in other galaxies has the same reddening properties as dust in the Milky Way but not if the reddening parameter is significantly reduced. Hicken et al. 88 find no evidence for a Hubble bubble at $z=0.024$ if the reddening parameter is set to $R_{V}=1.7$.

Our results suggest that the combination of the boost to the rest frame of the $\mathrm{CMB}$ compensating for structures in the range $30 h^{-1} \lesssim r \lesssim 62 h^{-1} \mathrm{Mpc}$, together with the treatment of parameters such as $R_{V}$ as adjustable in light curve reduction, may contribute significantly to the systematic uncertainties associated with SneIa. Reddening by dust in other galaxies is after all a physical quantity which should be determined independently of SneIa. Ideally it should not be treated as a parameter which one can freely adjust to obtain the best fit of Hubble residuals.

This issue has been studied independently by Finkelman et al. [93, 94] who investigated dust lanes in $15 \mathrm{E} / \mathrm{S} 0$ galaxies and determined extinction properties by fitting model galaxies to the unextinguished parts of the images in each of six spectral bands, and then subtracting these from the actual images. They found an average value $R_{V}=2.82 \pm 0.38$ for 8 galaxies in their first study [93], and $R_{V_{0}}=2.71 \pm 0.43$ for 7 galaxies in their second investigation [94]. For the combined sample $R_{V}=2.77 \pm 0.41$. This value is a little lower than the Milky Way value $R_{V}=3.1$ but consistent with it within the uncertainty.

Our results suggest that the convergence scale $z_{\text {conv }} \simeq$ 0.022 is close to that of the Hubble bubble originally proposed by Zehavi et al. [91], but the magnitude of the Hubble bubble effect is smaller when viewed in the LG frame. For consistency MLCS2k2 SneIa data should be reduced using $R_{V}$ values consistent with independent determinations, e.g., $R_{V}=2.77 \pm 0.41$ as suggested by the work of
Finkelman et al. [93, 94]. As discussed in Ref. 66] this is also important for cosmological model comparison.

Since the First Amendment SneIa data of Ref. 24] was reduced with $R_{V}=1.7$, one should check to what extent the difference in the amplitude of the bulk flow velocity from that of the COMPOSITE sample of [18] is due to the choice of the $R_{V}$ parameter. We suggest that the data set of Ref. [24] should be reanalysed with $R_{V}=$ $2.77 \pm 0.41$, and by the method of Sec. II in the LG frame.

\section{The asymptotic global Hubble constant}

The COMPOSITE sample enables us to determine the relative Hubble flow, but does not constrain the overall normalization of the distance scale and consequently the precise value of the global asymptotic Hubble constant.

The Hubble constant has recently been determined to high accuracy by the SH0ES survey as $H_{0}=73.8 \pm$ $2.4 \mathrm{kms}^{-1} \mathrm{Mpc}^{-1}$ [95]. Independent estimates of the Hubble constant using BAO data at a variety of redshifts [96 98], have yielded values $H_{0}=\{68.2 \pm 2.2,67 \pm$ $3.2,68.1 \pm 1.7\} \mathrm{km} \mathrm{s}^{-1} \mathrm{Mpc}^{-1}$ respectively in the (possibly curved) $\Lambda \mathrm{CDM}$ model. The Planck fit of the $\mathrm{CMB}$ anisotropies to a spatially flat $\Lambda \mathrm{CDM}$ model yields 72 . $H_{0}=67.4 \pm 1.4 \mathrm{~km} \mathrm{~s}^{-1} \mathrm{Mpc}^{-1}$. While these $H_{0}$ values are consistent with Ref. [95] at the $2 \sigma$ level, a further increase in precision could lead to tension. The BAO and Planck analyses rely on fits to the $\Lambda \mathrm{CDM}$ model at large redshifts, whereas the SHOES survey is less model dependent but relies on an empirical ladder of cosmic distance indicators on very nearby scales.

If we identify the cosmic rest frame with that of minimum Hubble flow variance, then the impact of performing all cosmological tests in such a frame rather than in the CMB frame needs to be carefully considered. The impact is likely to be most significant on those tests which directly use measurements on $z \lesssim 0.022$ scales. Whether this has an impact on measurements that establish the cosmic distance ladder is an intriguing question which should be investigated once the minimum Hubble flow variance rest frame is positively identified.

\section{E. Large angle CMB anomalies}

There are several observations concerning the large angle multipoles of the CMB anisotropy spectrum, which may be considered anomalous to varying degrees of statistical significance. These include: (i) the power asymmetry between the northern and southern hemispheres [28, 30, 31, 99]; (ii) the low quadrupole power 28, 100]; (iii) the alignment of the quadrupole and octupole [29, 100 102]; and (iv) the parity asymmetry [32]. The significance of some of these problems has increased with the recent release of Planck satellite data [33].

It is beyond the scope of the present paper to investigate all these anomalies. However, it is clear that 
our proposal to revisit a significant feature of the $\mathrm{CMB}$ anisotropy analysis, namely the nature of the dipole, will introduce systematics which would necessitate a reassessment of all of these issues.

There are two obvious potential lines of inquiry:

- The propagation of photons through the foregrounds contributing to the Hubble flow variance may produce a multipole signature which differs subtly from the pure dipole signature (11) associated with a Lorentz boost;

- Since the dipole subtraction is an integral part of the map-making procedure, differences in dipole subtraction may lead to subtle differences in the cleaning of galactic foregrounds [28].

A study by Freeman et al. [103] found that of several possible systematic errors, a 1-2\% error in the CMB dipole subtraction stood out as being an effect which could potentially resolve the power asymmetry anomaly.

We note that while a 1-2\% change in the dipole would not affect the power on small angles, its effect on the large angle multipoles would require a redrawing of the CMB sky maps. Any such redrawing may potentially alter other large angle features, such as the Cold Spot.

\section{F. The dark flow}

The determination of peculiar velocities via the kinematic Sunyaev-Zel'dovich (kSZ) effect [20, 21, 49, 104, 105] is particularly interesting, since it is a method which uses the CMB anisotropy spectrum, rather than directly testing the distance-redshift relation. Furthermore, the very large bulk flow that has been claimed by Kashlinsky et al. 20, 21] has been controversial. The effect was not reproduced in the subsequent studies of Keisler [106] and Osborne et al. [107]. However, Atrio-Barandela et al. 108, 109] showed that these differences could be attributed to flaws in the filtering methodology of their critics. Following the first release of Planck data, the debate has continued [110, 111].

A significant feature of the measurements of Kashlinsky et al. [20, 21, 49] is the claim that the result is independent of many systematics, since both foreground and cosmological dipoles and quadrupoles have been subtracted in a consistent way. It is claimed that the peculiar velocities inferred are those of the galaxy clusters with respect to the CMB in their own rest frames. Nonetheless, the direction of the reported bulk "dark flow" on scales $0.12 \leq z \leq 0.3,(\ell, b)=\left(267^{\circ}, 34^{\circ}\right) \pm 15^{\circ}$, coincides closely with that of the residual $\mathrm{CMB}$ dipole in the LG rest frame, while its amplitude is consistent with the boost of $635 \pm 38 \mathrm{~km} \mathrm{~s}^{-1}$ that the LG would be required to have with respect to the CMB frame to account for the residual dipole in the standard framework. Given these coincidences all potential systematics need to be considered.
Our results suggest in particular, that if the CMB rest frame is to be identified as that of minimum Hubble flow variance then such a frame is closer to that of the LG than to the conventionally assumed rest frame. We are unable to identify any obvious systematic errors in the highly technical analysis of Ref. [49]. However, they do of course implicitly assume that the conventional CMB frame is our actual relevant cosmic rest frame, an assumption which is not questioned in the standard framework but which our analysis leads us to question.

This issue might be resolved by carefully reperforming the analysis of Refs. [20, 21, 49] with each step in the pipeline that implicitly or explicitly assumes a normalization to the $\mathrm{CMB}$ frame redone as if the observation was made by observers in the LG frame. In particular, in the case of the WMAP data this should be done before applying the Wiener filter, and in the case of the $\mathrm{X}$-ray clusters this should be done before any calibration of any quantity which depends on redshift. If the frame transformation has any consequences for the cluster redshifts this would be likely to affect the nearer sample more; however, an overall boost (11) of the CMB sky might affect the whole analysis. One should begin with raw skymaps in which only the dipole and all higher order multipoles which correspond to a boost from the heliocentric to LG frame have been removed. This will of course leave a global dipole that will be removed in the data analysis pipeline. The more pertinent issue is whether starting in the LG frame changes the way in which the SZ components are treated by the Wiener filter. The intrinsic optical depths, $\tau$, of the clusters are convolved with the filter in the process of estimating the final effective $\tau$, which is a crucial physical quantity in the $\mathrm{kSZ}$ determination. A transformation to the LG frame may therefore potentially affect the result.

The intrinsic kSZ effect is of course due to the local CMB dipole at the cluster location. With our interpretation, this temperature dipole will include contributions from both a peculiar velocity and from the differential expansion of space due to inhomogeneities in the vicinity of the galaxy cluster. Estimates of the maximum possible Hubble flow variance based on void/wall statistics should therefore put bounds on the magnitude of what is assigned to individual "peculiar velocities" in the standard framework.

\section{G. Direct tests of a nonkinematic dipole}

It is possible to directly test the extent to which the $\mathrm{CMB}$ dipole is kinematic by considering the combined effects of the frequency shift plus aberration that arise from the local boost of an observer who views the spectrum of an otherwise isotropic background of sources [112].

The Planck satellite team has recently examined the effects of Doppler boosting on the primary CMB anisotropies, and claims evidence in favour of the standard boost interpretation [113]. It is notable, however, their results agree with the conventional boost direction, 
$(\ell, b)=\left(264^{\circ}, 48^{\circ}\right)$ only if small angle multipoles are included in the analysis, with a definitive measurement for multipoles in the range $l_{\min }=500<l<l_{\max }=2000$. If large angle multipoles are included and $l_{\max }$ is reduced to $l_{\max }<100$, then the inferred boost direction moves across the sky to coincide with the modulation dipole anomaly direction [31], $(\ell, b)=\left(224^{\circ},-22^{\circ}\right) \pm 24^{\circ}$.

This provides evidence that a nonkinematic boost component and the large angle anomalies may be related, as pointed out in Sec.VIE In Sec. IIIB we found there is no statistically significant CMB frame dipole in shell 4 with $\bar{r}=44.5 h^{-1} \mathrm{Mpc}$, within the range $40 \lesssim r \lesssim 60 h^{-1} \mathrm{Mpc}$ for which the CMB frame also has a smaller monopole variation in $H_{0}$ than the $\mathrm{LG}$ frame. Thus the observed dipole has features very close those of a Lorentz boost dipole, which smooth the Hubble flow at a particular radial scale, but not exactly. Work needs to be done to establish whether the angular scale dependence of Ref. [113] can be explained in terms of a component of the dipole, with properties close to but not exactly those produced by a boost.

The effects of aberration and frequency modulation can also be readily tested in the radio spectrum. In recent work Rubart and Schwarz [114] have found that the assumption of kinematic origin for the cosmic radio dipole is inconsistent at the $99.5 \%$ confidence level, using the NRAO VLA Sky Survey (NVSS). Furthermore, through a careful analysis of the biases introduced by different statistical estimators Rubart and Schwarz [114] have been able to resolve apparently discrepant results [115 117] from previous studies.

The direction of the radio dipole in NVSS is found to be $(\mathrm{RA}, \operatorname{dec})=\left(154^{\circ} \pm 21^{\circ},-2^{\circ} \pm 21^{\circ}\right)$ [114] in equatorial coordinates (epoch J2000). By comparison, in Sec. IIIB the smoothed dipole in the LG frame was found to have a direction $\left(180+\ell_{d},-b_{d}\right)=\left(263^{\circ} \pm 6^{\circ}, 39^{\circ} \pm 3^{\circ}\right)$ for the spheres $r>r_{o}$ with $20 h^{-1} \lesssim r_{o} \lesssim 45 h^{-1} \mathrm{Mpc}$ which produced a strong amplitude dipole. In equatorial coordinates this corresponds to $(\mathrm{RA}, \mathrm{dec})=\left(162^{\circ} \pm 4^{\circ},-14^{\circ} \pm\right.$ $3^{\circ}$ ), which lies well within the error circle of the NVSS radio dipole. Since the directions are consistent, the nonkinematic nature of the dipole found by Rubart and Schwarz [114] provides independent evidence for our hypothesis of Sec. V.

The NVSS data is of course sampled over much larger distances than the COMPOSITE sample, with a mean redshift $z \sim 1$, and even if the radio dipole is nonkinematic it could in principle be generated by a completely different effect to the foregrounds we have identified. While there are numerous systematic uncertainties which complicate the analysis, future surveys such as the Square Kilometre Array should have the power to potentially falsify the hypothesis of Sec. V. In particular, it should be possible to eventually determine just what fraction of the radio dipole is kinematic, along with the magnitude and direction of the associated boost.

\section{H. Conclusion}

While much work remains to be done, our results suggest that a fundamental revision of the treatment of peculiar velocities may shed new light on many of the puzzles raised by bulk flows, and perhaps even resolve some of the associated anomalies. If a large fraction of the $\mathrm{CMB}$ dipole is due to a residual anisotropy in the distanceredshift relation, as our results seem to suggest, then this may also have important consequences for many aspects of theoretical and observational cosmology.

Peculiar velocities will always play a role in observational cosmology - galaxies in clusters exhibit peculiar velocities with respect to the cluster barycentres, and this is directly observed in the "fingers of god" redshift space distortions. However, the most natural conclusion of our work is that on scales larger than gravitationally bound systems the variance of the Hubble flow should be treated as the differential expansion of regions of varying density, which have decelerated by different amounts from the initial uniform distribution at the epoch of last scattering. One should only talk about "infall" if the physical distance between objects is actually decreasing with time, rather than applying it to the case of denser regions which are expanding slower than the average. While directional forces are the basis of Newtonian mechanics in Euclidean space, there is nothing in general relativity which demands that such notions should apply to scales of tens of megaparsecs over which space is expanding.

In recent years there has been some discussion about whether it is conceptually more correct to think of space as expanding, or whether the treatment of the expansion by a simple Doppler law on a fixed background is sufficient [118 125]. In particular, Abramowicz et al. [118] showed that the expansion of space can in principle be observationally determined. Some other authors, e.g., Bunn and Hogg 123], maintained that the Doppler law picture is still useful. The debate involved thought experiments conducted within homogeneous isotropic cosmological models. Our results suggest that, as far as actual observations are concerned, variance in the Hubble law over scales of tens of megaparsecs cannot be simply reduced to a boost at a point; space really is expanding, and by differential amounts.

Acknowledgements This work was supported by the Marsden Fund of the Royal Society of New Zealand. We are especially grateful to Krzysztof Bolejko for assistance with ray tracing studies. We also thank Anthony Brown, Chris Gordon, Loretta Dunne, Steve Maddox and Maria Mattsson for helpful discussions, and Fernando AtrioBarandela and Alexander Kashlinsky for correspondence. 


\begin{tabular}{|c|c|c|c|c|c|c|c|c|c|c|c|}
\hline Shell $s$ & 1 & 2 & 3 & 4 & 5 & 6 & 7 & 8 & 9 & 10 & 11 \\
\hline$N_{s}$ & 78 & 457 & 494 & 713 & 799 & 555 & 413 & 303 & 221 & 274 & 91 \\
\hline$r_{s}\left(h^{-1} \mathrm{Mpc}\right)$ & 2.00 & 12.50 & 25.00 & 37.50 & 50.00 & 62.50 & 75.00 & 87.50 & 100.00 & 112.50 & 156.25 \\
\hline $\bar{r}_{s}\left(h^{-1} \mathrm{Mpc}\right)$ & 7.23 & 16.22 & 30.12 & 44.52 & 55.11 & 69.25 & 81.06 & 93.68 & 105.10 & 126.51 & 182.59 \\
\hline$\left(H_{s}\right)_{\mathrm{CMB}}$ & 152.7 & 109.5 & 108.2 & 103.5 & 101.4 & 103.0 & 102.0 & 103.1 & 104.1 & 102.1 & 100.1 \\
\hline$\left(\bar{\sigma}_{s}\right)_{\mathrm{CMB}}$ & 4.9 & 1.5 & 1.1 & 0.7 & 0.7 & 0.7 & 0.9 & 0.9 & 1.0 & 0.8 & 1.7 \\
\hline$\left(Q_{s}\right)_{\mathrm{CMB}}$ & 0.000 & 0.000 & 0.024 & 1.000 & 1.000 & 1.000 & 1.000 & 1.000 & 1.000 & 1.000 & 0.999 \\
\hline$\left(\chi^{2} / \nu\right)_{\mathrm{CMB}}$ & 9.092 & 1.818 & 1.130 & 0.739 & 0.606 & 0.664 & 0.580 & 0.577 & 0.603 & 0.685 & 0.581 \\
\hline$\left(H_{s}\right)_{\mathrm{LG}}$ & 107.8 & 98.2 & 103.7 & 105.4 & 103.6 & 101.4 & 102.7 & 103.5 & 103.4 & 102.4 & 101.0 \\
\hline$\left(\bar{\sigma}_{s}\right)_{\mathrm{LG}}$ & 3.4 & 1.4 & 1.0 & 0.8 & 0.7 & 0.7 & 0.9 & 0.9 & 1.0 & 0.8 & 1.7 \\
\hline$\left(Q_{s}\right)_{\mathrm{LG}}$ & 0.000 & 0.000 & 0.000 & 0.980 & 1.000 & 1.000 & 1.000 & 1.000 & 1.000 & 1.000 & 0.999 \\
\hline$\left(\chi^{2} / \nu\right)_{\mathrm{LG}}$ & 4.048 & 2.340 & 1.431 & 0.894 & 0.583 & 0.723 & 0.578 & 0.513 & 0.595 & 0.667 & 0.590 \\
\hline $\ln B\left(r \geq r_{s}\right)$ & 50.84 & 11.42 & 5.97 & 1.04 & 1.58 & 2.16 & 1.53 & 1.67 & 1.52 & 0.44 & \\
\hline Shell $s$ & $1^{\prime}$ & $2^{\prime}$ & $3^{\prime}$ & $4^{\prime}$ & $5^{\prime}$ & $6^{\prime}$ & 7 & $8^{\prime}$ & $9^{\prime}$ & $10^{\prime}$ & 11 \\
\hline$N_{s}$ & 284 & 488 & 532 & 869 & 669 & 481 & 343 & 271 & 160 & 204 & 91 \\
\hline$r_{s}\left(h^{-1} \mathrm{Mpc}\right)$ & 6.25 & 18.75 & 31.25 & 43.75 & 56.25 & 68.75 & 81.25 & 93.75 & 106.25 & 118.75 & 156.25 \\
\hline $\bar{r}_{s}\left(h^{-1} \mathrm{Mpc}\right)$ & 12.25 & 24.05 & 37.47 & 49.17 & 61.75 & 73.92 & 87.15 & 99.13 & 111.81 & 131.52 & 182.59 \\
\hline$\left(H_{s}\right)_{\mathrm{CMB}}$ & 119.5 & 107.3 & 105.3 & 102.4 & 103.0 & 102.2 & 102.7 & 103.9 & 101.7 & 102.3 & 100.1 \\
\hline$\left(\bar{\sigma}_{s}\right)_{\mathrm{CMB}}$ & 2.2 & 1.2 & 1.0 & 0.7 & 0.8 & 0.8 & 0.9 & 0.9 & 1.1 & 0.9 & 1.7 \\
\hline$\left(Q_{s}\right)_{\mathrm{CMB}}$ & 0.000 & 0.000 & 0.649 & 1.000 & 1.000 & 1.000 & 1.000 & 1.000 & 1.000 & 1.000 & 0.999 \\
\hline$\left(\chi^{2} / \nu\right)_{\mathrm{CMB}}$ & 4.042 & 1.313 & 0.975 & 0.645 & 0.638 & 0.587 & 0.624 & 0.604 & 0.561 & 0.690 & 0.581 \\
\hline$\left(H_{s}\right)_{\mathrm{LG}}$ & 99.7 & 101.1 & 103.6 & 105.7 & 102.7 & 101.6 & 103.2 & 103.6 & 101.5 & 102.7 & 101.0 \\
\hline$\left(\bar{\sigma}_{s}\right)_{\mathrm{LG}}$ & 1.8 & 1.1 & 0.9 & 0.7 & 0.8 & 0.8 & 0.9 & 0.9 & 1.0 & 0.9 & 1.7 \\
\hline$\left(Q_{s}\right)_{\mathrm{LG}}$ & 0.000 & 0.000 & 0.001 & 1.000 & 1.000 & 1.000 & 1.000 & 1.000 & 1.000 & 1.000 & 0.999 \\
\hline$\left(\chi^{2} / \nu\right)_{\mathrm{LG}}$ & 3.581 & 1.496 & 1.197 & 0.699 & 0.653 & 0.650 & 0.592 & 0.552 & 0.563 & 0.655 & 0.590 \\
\hline $\ln B\left(r \geq r_{s}\right)$ & 23.97 & 6.29 & 2.31 & 0.59 & 1.86 & 1.53 & 1.32 & 1.24 & 0.57 & 0.42 & \\
\hline
\end{tabular}

TABLE IV: The analysis of Table $\llbracket$ is repeated removing all points which contribute a value $\chi^{2}>5$ in both the CMB and LG frames in both the primed and unprimed shells. A total 4398 points remain.

\begin{tabular}{|c|c|c|c|c|c|c|c|c|c|c|c|}
\hline Shell $s$ & 1 & 2 & 3 & 4 & 5 & 6 & 7 & 8 & 9 & 10 & 11 \\
\hline$N_{s}$ & 56 & 385 & 454 & 692 & 788 & 548 & 410 & 301 & 217 & 270 & 91 \\
\hline$r_{s}\left(h^{-1} \mathrm{Mpc}\right)$ & 2.00 & 12.50 & 25.00 & 37.50 & 50.00 & 62.50 & 75.00 & 87.50 & 100.00 & 112.50 & 156.25 \\
\hline $\bar{r}_{s}\left(h^{-1} \mathrm{Mpc}\right)$ & 9.04 & 17.87 & 30.33 & 44.58 & 55.10 & 68.74 & 80.95 & 93.74 & 105.08 & 126.20 & 182.59 \\
\hline$\left(H_{s}\right)_{\mathrm{CMB}}$ & 155.4 & 109.3 & 107.6 & 103.0 & 101.4 & 103.9 & 102.0 & 103.4 & 104.6 & 102.6 & 100.1 \\
\hline$\left(\bar{\sigma}_{s}\right)_{\mathrm{CMB}}$ & 5.0 & 1.6 & 1.2 & 0.8 & 0.7 & 0.8 & 0.9 & 0.9 & 1.0 & 0.8 & 1.7 \\
\hline$\left(Q_{s}\right)_{\mathrm{CMB}}$ & 0.000 & 0.228 & 0.991 & 1.000 & 1.000 & 1.000 & 1.000 & 1.000 & 1.000 & 1.000 & 0.999 \\
\hline$\left(\chi^{2} / \nu\right)_{\mathrm{CMB}}$ & 2.895 & 1.053 & 0.850 & 0.632 & 0.546 & 0.616 & 0.550 & 0.538 & 0.498 & 0.613 & 0.581 \\
\hline$\left(H_{s}\right)_{\mathrm{LG}}$ & 114.3 & 101.3 & 104.7 & 105.0 & 103.5 & 102.8 & 102.9 & 103.6 & 103.7 & 102.8 & 101.0 \\
\hline$\left(\bar{\sigma}_{s}\right)_{\mathrm{LG}}$ & 3.6 & 1.5 & 1.1 & 0.8 & 0.7 & 0.8 & 0.9 & 0.9 & 1.0 & 0.8 & 1.7 \\
\hline$\left(Q_{s}\right)_{\mathrm{LG}}$ & 0.000 & 0.529 & 0.778 & 1.000 & 1.000 & 1.000 & 1.000 & 1.000 & 1.000 & 1.000 & 0.999 \\
\hline$\left(\chi^{2} / \nu\right)_{\mathrm{LG}}$ & 2.275 & 0.993 & 0.949 & 0.745 & 0.523 & 0.647 & 0.549 & 0.482 & 0.519 & 0.606 & 0.590 \\
\hline $\ln B\left(r \geq r_{s}\right)$ & 50.81 & 10.83 & 5.31 & 1.78 & 2.40 & 3.02 & 1.99 & 2.19 & 1.98 & 0.59 & \\
\hline Shell $s$ & $1^{\prime}$ & $2^{\prime}$ & $3^{\prime}$ & $4^{\prime}$ & $5^{\prime}$ & 6 ' & $7^{\prime}$ & $8^{\prime}$ & $9^{\prime}$ & $10^{\prime}$ & 11 \\
\hline$N_{s}$ & 223 & 436 & 501 & 856 & 657 & 477 & 339 & 268 & 158 & 201 & 91 \\
\hline$r_{s}\left(h^{-1} \mathrm{Mpc}\right)$ & 6.25 & 18.75 & 31.25 & 43.75 & 56.25 & 68.75 & 81.25 & 93.75 & 106.25 & 118.75 & 156.25 \\
\hline $\bar{r}_{s}\left(h^{-1} \mathrm{Mpc}\right)$ & 12.92 & 24.39 & 37.80 & 49.23 & 61.51 & 73.84 & 87.08 & 99.03 & 111.89 & 131.38 & 182.59 \\
\hline$\left(H_{s}\right)_{\mathrm{CMB}}$ & 124.5 & 106.4 & 105.7 & 102.3 & 102.9 & 103.0 & 103.0 & 104.0 & 101.9 & 103.1 & 100.1 \\
\hline$\left(\bar{\sigma}_{s}\right)_{\mathrm{CMB}}$ & 2.4 & 1.3 & 1.0 & 0.7 & 0.8 & 0.8 & 0.9 & 0.9 & 1.1 & 0.9 & 1.7 \\
\hline$\left(Q_{s}\right)_{\mathrm{CMB}}$ & 0.000 & 0.994 & 1.000 & 1.000 & 1.000 & 1.000 & 1.000 & 1.000 & 1.000 & 1.000 & 0.999 \\
\hline$\left(\chi^{2} / \nu\right)_{\mathrm{CMB}}$ & 2.414 & 0.839 & 0.794 & 0.588 & 0.568 & 0.556 & 0.568 & 0.554 & 0.486 & 0.610 & 0.581 \\
\hline$\left(H_{s}\right)_{\mathrm{LG}}$ & 105.2 & 101.3 & 104.4 & 105.3 & 102.9 & 102.9 & 103.5 & 103.6 & 101.6 & 103.4 & 101.0 \\
\hline$\left(\bar{\sigma}_{s}\right)_{\mathrm{LG}}$ & 2.0 & 1.2 & 1.0 & 0.7 & 0.8 & 0.8 & 0.9 & 0.9 & 1.1 & 0.9 & 1.7 \\
\hline$\left(Q_{s}\right)_{\mathrm{LG}}$ & 0.000 & 0.863 & 0.972 & 1.000 & 1.000 & 1.000 & 1.000 & 1.000 & 1.000 & 1.000 & 0.999 \\
\hline$\left(\chi^{2} / \nu\right)_{\mathrm{LG}}$ & 1.444 & 0.926 & 0.883 & 0.616 & 0.566 & 0.588 & 0.539 & 0.506 & 0.510 & 0.591 & 0.590 \\
\hline $\ln B\left(r \geq r_{s}\right)$ & 29.83 & 5.66 & 2.75 & 1.08 & 2.29 & 2.01 & 1.66 & 1.59 & 0.83 & 0.62 & \\
\hline
\end{tabular}

TABLE V: The analysis of Table $\prod$ is repeated removing all points which contribute a value $\chi^{2}>5$ in either the CMB or LG frames in both the primed and unprimed shells. A total 4212 points remain. 


\section{Appendix A: Nonlinearity from foreground structures and statistical issues}

We have investigated the extent to which the relative magnitude of the Bayes factors in Fig. 4 is driven by the greater scatter relative to a linear law, rather than by the difference of the linear fit of the Hubble constant from its asymptotic value. To investigate this in Tables IV D and VI we have repeated the analysis of Secs. IIA IIB that led to Table【 by successively removing points which contribute the greatest scatter:

(1) Firstly, in Table IV we remove points which contribute an individual value $\chi_{i, s}^{2} \equiv\left(r_{i}-c z_{i} / H_{s}\right)^{2} / \sigma_{i}^{2}$ with $\chi_{i, s}^{2}>5$ in both the CMB and LG frames for both the primed and unprimed choices of shell boundaries.

(2) Secondly, in Table $\nabla$ we remove points with $\chi_{i, s}^{2}>5$ in either the CMB or LG frame for both choices of shell boundaries.

(3) Finally, in Table V1 we remove points with $\chi_{i, s}^{2}>5$ in either the CMB or LG frame for either choice of shell boundaries.

Since the underlying Hubble relation is not linear, it is clear that we are rejecting some of the points with the strongest discriminating power in such a procedure. Indeed, only the points excluded in Table IV] could be said to be outliers in any sense ${ }^{20}$ since all other points have $\chi_{i, s}^{2}<5$ in at least one frame and shell slicing.

Nonetheless, although this procedure is not a perfect one it does illustrate that as the linear goodness of fit is improved the relative Bayes factor is somewhat reduced but still remains significant. In Table IV only shell 4 has a significantly improved goodness of fit in both frames. However, in Table $\mathrm{V}$ the shells $\left\{2,2^{\prime}, 3,3^{\prime}\right\}$ now all have an acceptable goodness of fit and a reduced $\chi^{2}$ close to unity. Although $\ln B$ is somewhat reduced, a very strong value $\ln B>5$ is still found in shells $\left\{2,2^{\prime}, 3\right\}$ and indeed in shell 2 we still have $\ln B>10$. Our statistical conclusions are thus robust.

In the final Table VI even shells 1 and $1^{\prime}$ have an acceptable goodness of fit. However, for shell 1 this comes at the expense of having removed two thirds of the 92 points originally present. The $12.5 h^{-1} \mathrm{Mpc}$ radius of shell 1 is simply so small relative to the foreground structures that a notion of a spherically averaged linear Hubble law is not really applicable. However, our statistical conclusions do not rely on the innermost shell.

20 The points excluded in Table IV are still only outliers relative to the CMB and LG frames. Since we have not yet established a "minimum variance Hubble frame", it is perfectly possible that some of the points so excluded in fact have little scatter relative to a linear Hubble law in some other minimum variance frame.
A robust optimization procedure could also be used to estimate $H_{s}$ in the inner shells. However, that would also require modeling the nonlinearity of the inner shells, and in the present paper we aim to be model-independent, without any particular theoretical biases. To this end, we believe the very strong evidence for the relative uniformity of the LG and LS frames as compared to the CMB frame, has been clearly demonstrated.

\section{Appendix B: Angular Gaussian window averages}

In Sec. III] we follow McClure and Dyer [25] (MD07) to produce contour maps of the angular variation in the Hubble flow. At each grid point on the sky, a mean $H_{\alpha}$ is calculated in which the value of $c z_{i} / r_{i}$ for each data point is weighted according to its angular separation from the grid point. The $i$ th data point is weighted by

$$
W_{i \alpha}=\frac{1}{\sqrt{2 \pi} \sigma_{\theta}} \exp \left(\frac{-\theta_{i}^{2}}{2 \sigma_{\theta}^{2}}\right),
$$

where $\cos \theta_{i}=\vec{r}_{\text {grid }} \cdot \overrightarrow{r_{i}}, \sigma_{\theta}=25^{\circ}$ is the smoothing scale and the Greek subscript $\alpha$ is used to represent the angular dependence on the spherical polar coordinates, $(\ell, b)$ encoded in $\theta_{i}$.

The choice of smoothing scale, $\sigma_{\theta}$, is constrained by the fact that the diameter $2 \sigma_{\theta}$ of the Gaussian window function should be wider than the angular width of the Zone of Avoidance $(\mathrm{ZoA})$. If it were smaller then windows centred on gridpoints close to the galactic plane would have insufficient data to give reliable results in those regions. The $\mathrm{ZoA}$ is typically $30^{\circ}$ wide for the COMPOSITE sample, which means that the smoothing scale must be greater than $15^{\circ}$. On the other hand we cannot make the smoothing scale so large that we lose all angular resolution. This determines the choice $\sigma_{\theta}=25^{\circ}$, which matches that made by MD07. This angle subtends an area 0.59 steradians, $4.8 \%$ of the full sky. We have checked that varying the smoothing scale in the range $15^{\circ}<\sigma_{\theta}<40^{\circ}$ does not significantly change our results.

Since (B1) determines a mean value of $H_{\alpha}$ at each grid point on the sky in which each data point is weighted by its distance from the grid point, there will be larger uncertainties for grid points near the ZoA, as can be seen in Fig. 13.

In the method adopted by MD07, the weighted mean $H_{\alpha}$ is calculated at each spherical polar grid point by

$$
H_{\alpha}=\frac{\sum_{i=1}^{N} W_{i \alpha} c z_{i} r_{i}^{-1}}{\sum_{j=1}^{N} W_{j \alpha}},
$$

with the weight (B1). The variance of this sample mean at each grid point is given by

$$
\bar{\sigma}_{\alpha}^{2}=\frac{\sum_{i=1}^{N} W_{i \alpha}^{2} \sigma_{H_{i}}^{2}}{\left(\sum_{j=1}^{N} W_{j \alpha}\right)^{2}},
$$




\begin{tabular}{|c|c|c|c|c|c|c|c|c|c|c|c|}
\hline Shell $s$ & 1 & 2 & 3 & 4 & 5 & 6 & 7 & 8 & 9 & 10 & 11 \\
\hline$N_{s}$ & 30 & 366 & 447 & 691 & 786 & 547 & 410 & 301 & 217 & 270 & 91 \\
\hline$r_{s}\left(h^{-1} \mathrm{Mpc}\right)$ & 2.00 & 12.50 & 25.00 & 37.50 & 50.00 & 62.50 & 75.00 & 87.50 & 100.00 & 112.50 & 156.25 \\
\hline $\bar{r}_{s}\left(h^{-1} \mathrm{Mpc}\right)$ & 8.44 & 18.32 & 30.20 & 44.58 & 55.10 & 68.74 & 80.95 & 93.74 & 105.08 & 126.20 & 182.59 \\
\hline$\left(H_{s}\right)_{\mathrm{CMB}}$ & 138.4 & 107.2 & 107.1 & 103.0 & 101.2 & 103.8 & 102.0 & 103.4 & 104.6 & 102.6 & 100.1 \\
\hline$\left(\bar{\sigma}_{s}\right)_{\mathrm{CMB}}$ & 6.3 & 1.6 & 1.2 & 0.8 & 0.7 & 0.8 & 0.9 & 0.9 & 1.0 & 0.8 & 1.7 \\
\hline$\left(Q_{s}\right)_{\mathrm{CMB}}$ & 0.320 & 0.992 & 0.997 & 1.000 & 1.000 & 1.000 & 1.000 & 1.000 & 1.000 & 1.000 & 0.999 \\
\hline$\left(\chi^{2} / \nu\right)_{\mathrm{CMB}}$ & 1.103 & 0.829 & 0.823 & 0.627 & 0.531 & 0.611 & 0.550 & 0.538 & 0.498 & 0.613 & 0.581 \\
\hline$\left(H_{s}\right)_{\mathrm{LG}}$ & 106.9 & 101.7 & 104.2 & 104.9 & 103.4 & 102.7 & 102.9 & 103.6 & 103.7 & 102.8 & 101.0 \\
\hline$\left(\bar{\sigma}_{s}\right)_{\mathrm{LG}}$ & 4.9 & 1.5 & 1.1 & 0.8 & 0.7 & 0.8 & 0.9 & 0.9 & 1.0 & 0.8 & 1.7 \\
\hline$\left(Q_{s}\right)_{\mathrm{LG}}$ & 0.464 & 0.530 & 0.907 & 1.000 & 1.000 & 1.000 & 1.000 & 1.000 & 1.000 & 1.000 & 0.999 \\
\hline$\left(\chi^{2} / \nu\right)_{\mathrm{LG}}$ & 1.001 & 0.993 & 0.912 & 0.738 & 0.519 & 0.638 & 0.549 & 0.482 & 0.519 & 0.606 & 0.590 \\
\hline $\ln B\left(r \geq r_{s}\right)$ & 21.00 & 8.00 & 4.88 & 1.74 & 2.33 & 2.96 & 1.99 & 2.19 & 1.98 & 0.59 & \\
\hline Shell $s$ & $1^{\prime}$ & $2^{\prime}$ & $3^{\prime}$ & $4^{\prime}$ & $5^{\prime}$ & $6^{\prime}$ & $7^{\prime}$ & $8^{\prime}$ & $9^{\prime}$ & $10^{\prime}$ & 11 \\
\hline$N_{s}$ & 181 & 429 & 498 & 854 & 655 & 477 & 339 & 268 & 158 & 201 & 91 \\
\hline$r_{s}\left(h^{-1} \mathrm{Mpc}\right)$ & 6.25 & 18.75 & 31.25 & 43.75 & 56.25 & 68.75 & 81.25 & 93.75 & 106.25 & 118.75 & 156.25 \\
\hline $\bar{r}_{s}\left(h^{-1} \mathrm{Mpc}\right)$ & 14.27 & 24.55 & 37.83 & 49.22 & 61.49 & 73.84 & 87.08 & 99.03 & 111.89 & 131.38 & 182.59 \\
\hline$\left(H_{s}\right)_{\mathrm{CMB}}$ & 112.5 & 106.3 & 104.9 & 102.2 & 102.7 & 103.0 & 103.0 & 104.0 & 101.9 & 103.1 & 100.1 \\
\hline$\left(\bar{\sigma}_{s}\right)_{\mathrm{CMB}}$ & 2.3 & 1.3 & 1.0 & 0.7 & 0.8 & 0.8 & 0.9 & 0.9 & 1.1 & 0.9 & 1.7 \\
\hline$\left(Q_{s}\right)_{\mathrm{CMB}}$ & 0.504 & 0.999 & 1.000 & 1.000 & 1.000 & 1.000 & 1.000 & 1.000 & 1.000 & 1.000 & 0.999 \\
\hline$\left(\chi^{2} / \nu\right)_{\mathrm{CMB}}$ & 0.995 & 0.803 & 0.770 & 0.578 & 0.555 & 0.556 & 0.568 & 0.554 & 0.486 & 0.610 & 0.581 \\
\hline$\left(H_{s}\right)_{\mathrm{LG}}$ & 103.0 & 100.9 & 104.3 & 105.2 & 102.7 & 102.9 & 103.5 & 103.6 & 101.6 & 103.4 & 101.0 \\
\hline$\left(\bar{\sigma}_{s}\right)_{\mathrm{LG}}$ & 2.1 & 1.2 & 1.0 & 0.7 & 0.8 & 0.8 & 0.9 & 0.9 & 1.1 & 0.9 & 1.7 \\
\hline$\left(Q_{s}\right)_{\mathrm{LG}}$ & 0.243 & 0.966 & 0.980 & 1.000 & 1.000 & 1.000 & 1.000 & 1.000 & 1.000 & 1.000 & 0.999 \\
\hline$\left(\chi^{2} / \nu\right)_{\mathrm{LG}}$ & 1.071 & 0.879 & 0.875 & 0.610 & 0.555 & 0.588 & 0.539 & 0.506 & 0.510 & 0.591 & 0.590 \\
\hline $\ln B\left(r \geq r_{s}\right)$ & 11.34 & 4.80 & 2.07 & 1.03 & 2.22 & 2.01 & 1.66 & 1.59 & 0.83 & 0.62 & \\
\hline
\end{tabular}

TABLE VI: The analysis of Table $\$ is repeated removing all points which contribute a value $\chi^{2}>5$ in either the CMB or LG frames in either the primed or unprimed shells. A total 4156 points remain.

where

$$
\sigma_{H_{i}}=\frac{c z_{i} \sigma_{i}}{r_{i}^{2}}
$$

is the standard uncertainty from error propagation of the uncertainty $\sigma_{i}$ in the $i$ th distance $r_{i}$ in (B2) $-(\bar{B} 4)$ produced values.

No additional uncertainty weighting of the weight $W_{i \alpha}$ was used by MD07 in the determination of $H_{\alpha}$, since they claimed that the impact of the errors in the individual data points is averaged out by the Gaussian weighting procedure. The individual uncertainties in the COMPOSITE sample are larger, and therefore the question of the treatment of the uncertainty weightings in the determination of the mean (B2) is an important one.

In order to manage the uncertainty weightings, rather than using Eqs. (B2)-(B4), we will instead determine the weighted mean $H_{\alpha}$ at each spherical polar grid point $(\ell, b)$, by first evaluating its inverse

$$
H_{\alpha}^{-1}=\frac{\sum_{i=1}^{N} W_{i \alpha} r_{i}\left(c z_{i}\right)^{-1}}{\sum_{j=1}^{N} W_{j \alpha}}
$$

with the weight (B1). The variance of $H_{\alpha}^{-1}$ at each grid point is then given by

$$
\bar{\sigma}_{H_{\alpha}^{-1}}^{2}=\frac{\sum_{i=1}^{N} W_{i \alpha}^{2} \sigma_{H_{i}^{-1}}^{2}}{\left(\sum_{j=1}^{N} W_{j \alpha}\right)^{2}},
$$

where

$$
\sigma_{H_{i}^{-1}}=\frac{\sigma_{i}}{c z_{i}}
$$

is the standard uncertainty in $H_{i}^{-1}=r_{i} /\left(c z_{i}\right)$. Then

$$
\bar{\sigma}_{\alpha}=\bar{\sigma}_{H_{\alpha}^{-1}} H_{\alpha}^{2}
$$

is the standard uncertainty in $H_{\alpha}$. If, following MD07, no additional uncertainty weightings are used then in (B5) $W_{i \alpha}$ is given by (B1). Alternatively, if inverse variance (IV) uncertainty weightings are used then we replace (B1) by

$$
W_{i \alpha}=\frac{1}{\sigma_{H_{i}^{-1}}^{2} \sqrt{2 \pi} \sigma_{\theta}} \exp \left(\frac{-\theta_{i}^{2}}{2 \sigma_{\theta}^{2}}\right) .
$$

The reason that it is preferable to work with $H_{\alpha}^{-1}$ is a consequence of the dominant uncertainties in the COMPOSITE sample being those associated with the distance measure, $\sigma_{i}$. In the case of the radial shells we chose to minimize $\sum_{i}\left[\sigma_{i}^{-1}\left(r_{i}-c z_{i} / H\right)\right]^{2}$ with respect to $H$ for the same reason. The Gaussian window averaging adds a nonlinear weighting to what is otherwise a linear regression. Using (B9) ensures that the nonlinear weighting is added to IV weightings determined from uncertainties (B7) which are themselves linear in the measurement 
uncertainties. The alternative procedure of (B2)-(B4) introduces a different distance weighting of each point in (B4) which leads to different results ${ }^{21}$ when combined with the Gaussian window averaging in an equation analogous to (B9).

Use of a standard IV weighting in (B9) may not be the most robust method for uncertainty estimates for this data set. In particular, as has been discussed in WFH09 and FWH10 in the standard peculiar velocity framework the nonideal geometry of typical surveys can lead to an aliasing of small scale power. Where the data is sparse biases can be introduced relative to the bulk flow of a regular volume that one is ideally interested in. To deal with these issues Watkins, Feldman and Hudson [18, 19] have developed a minimum variance weighting method with respect to the leading peculiar velocity moment amplitudes (dipole, quadrupole and octupole). To treat these issues in our framework requires the development of a similar methodology, as is discussed in Sec. VIA and is left for future work.

McClure and Dyer 25] performed Monte Carlo simulations to assess the significance of the variation that they found. While a similar analysis has been undertaken by one of us [126], in the present paper we will use the more direct method of plotting the contours of the uncertainty $\bar{\sigma}_{\alpha}-$ as given by (B8) using either (B1) or (B9) - on the same map as the Hubble flow contours. Such a plot of the angular variation of the uncertainty contains much detailed information, uncertainties being larger in some angular regions rather than others.

\section{Uncertainties in Gaussian window averages}

Dealing with the uncertainties in the Gaussian window averaged sky maps is complicated if we wish to constrain individual large angle multipoles. Since we have not done a Fourier analysis on the raw data, we have avoided the direct problems of nonorthogonality of Fourier modes that arise from an analysis on a cut sky determined by the Zone of Avoidance. However, the finite area of the Gaussian window function may introduce its own systematic issues. This area is 0.59 steradians for $\sigma_{\theta}=25^{\circ}$, giving effectively 21.4 independent patches on the sky to constrain the 5 independent degrees of freedom that define the quadrupole, and the 7 that define the octupole.

Putting aside the question of the multipole decomposition, the overall uncertainties (B8) in the Gaussian window averages are readily represented as a function of angular position. In Fig. 13] we show the example of the

${ }^{21}$ We found that using Eqs. B2 - B4 in place of B5 - B7 gives results which differ very little from each other if IV weightings are not used. However, once IV weightings are included using (B2) - (B4) gives values of $H_{\alpha}$ with a mean which is $10 \%$ lower than the mean values determined from the averages in spherical shells. outer $r>15 h^{-1} \mathrm{Mpc}$ LG frame sky map, with the contours of the Hubble flow variance plotted as solid lines overlaid with colour map contours showing the angular uncertainties as a function of angular position on the sky. In each case we have used $\sigma_{\theta}=25^{\circ}$.

The angular uncertainties are somewhat greater in a curved band near the galactic plane. This effect is due to the absence of data in the ZoA as well as the propagation of measurement uncertainties through the Gaussian window averaging procedure. We have checked that the same band of greater uncertainties is obtained in the CMB frame map, even though the positions of the extrema are quite different in that case.

In Fig. 13 we identify the poles corresponding to the maximum and minimum Hubble variance in the $\mathrm{LG}$ frame, and plot a $1 \sigma$ contour around each pole, where the $1 \sigma$ value is taken as the maximum on the map, i.e., $1.02 h \mathrm{~km} \mathrm{~s}^{-1} \mathrm{Mpc}^{-1}$ in the unweighted case and $0.75 \mathrm{hms}^{-1} \mathrm{Mpc}^{-1}$ in the weighted case. In the unweighted case, we find $(\ell, b)=\left(116^{\circ},-35^{\circ}\right)$ and $(\ell, b)=$ $\left(249^{\circ}, 21^{\circ}\right)$ for the maximum and minimum respectively. Similarly, $(\ell, b)=\left(105^{\circ},-27^{\circ}\right)$ and $(\ell, b)=\left(253^{\circ}, 24^{\circ}\right)$ for the maximum and minimum in the IV weighted case. The poles are somewhat squeezed in galactic longitude as compared to a pure dipole ${ }^{22}$.

The mean value of $H_{0}$ obtained by Gaussian window averaging differs in general from the values obtained by spherical averages. For the $r>15 h^{-1} \mathrm{Mpc}$ LG frame Gaussian window average, for example, $H_{0}$ is $97.3 h \mathrm{~km} \mathrm{~s}^{-1} \mathrm{Mpc}^{-1}$ in the unweighted case and $102.6 \mathrm{hms}^{-1} \mathrm{Mpc}^{-1}$ in the IV weighted case. In the unweighted case the maximum and minimum values of $H_{\alpha}$ are $+9.3 \%$ and $-9.4 \%$ from the mean respectively. With IV weightings the differences are $+11.0 \%$ and $-7.9 \%$ respectively ${ }^{23}$. In each case these differences are considerably larger than the standard uncertainty at any angle, which is of order $1 \%$ of the mean $H_{0}$.

\section{Appendix C: Dipole estimation}

In Sec. III B we perform a $\chi^{2}$ minimization of the simple dipole Hubble law (10). For each radial shell we minimize $\chi^{2}=\sum_{i}\left[\sigma_{H_{i}}^{-1}\left(H_{i}-H_{d}-\boldsymbol{\beta} \cdot \mathbf{n}_{i}\right)\right]^{2}$, where $\boldsymbol{\beta} \equiv$ $\left(\beta_{x}, \beta_{y}, \beta_{z}\right)=\left(\beta \cos \ell_{d} \cos b_{d}, \beta \cos \ell_{d} \sin b_{d}, \beta \sin b_{d}\right)$, $\mathbf{n}_{i} \equiv\left(n_{x i}, n_{y i}, n_{z i}\right)=\left(\cos \ell_{i} \cos b_{i}, \cos \ell_{i} \sin b_{i}, \sin b_{i}\right)$, $H_{i}=c z_{i} / r_{i}$ and its uncertainty $\sigma_{H_{i}}$ is given by (B4). Minimization with respect to the four independent pa-

22 The latitude of the unweighted maximum matches that of the corresponding pure dipole law fit, $\left(\ell_{d}, b_{d}\right)=\left(68^{\circ} \pm 3,-38^{\circ} \pm 2^{\circ}\right)$ from Table VIII within $1 \sigma$, while the longitude of the minimum matches that of $\left(\ell_{d}+180^{\circ},-b_{d}\right)$.

23 The maximum variation is comparable to the ratio $\beta / H_{d}$ in Table VIII below, which is $12.0 \pm 0.5 \%$ for $r>15 h^{-1} \mathrm{Mpc}$. 
rameters $X^{a} \equiv\left(H_{d}, \beta_{x}, \beta_{y}, \beta_{z}\right)$ yields the linear system

$$
\begin{aligned}
& H_{d} \sum_{i} \frac{1}{\sigma_{H_{i}}^{2}}+\beta_{x} \sum_{i} \frac{n_{x i}}{\sigma_{H_{i}}^{2}}+\beta_{y} \sum_{i} \frac{n_{y i}}{\sigma_{H_{i}}^{2}} \\
& +\beta_{z} \sum_{i} \frac{n_{z i}}{\sigma_{H_{i}}^{2}}=\sum_{i} \frac{H_{i}}{\sigma_{H_{i}}^{2}} \\
& H_{d} \sum_{i} \frac{n_{x i}}{\sigma_{H_{i}}^{2}}+\beta_{x} \sum_{i} \frac{n_{x i}^{2}}{\sigma_{H_{i}}^{2}}+\beta_{y} \sum_{i} \frac{n_{x i} n_{y i}}{\sigma_{H_{i}}^{2}} \\
& +\beta_{z} \sum_{i} \frac{n_{x i} n_{z i}}{\sigma_{H_{i}}^{2}}=\sum_{i} \frac{H_{i} n_{x i}}{\sigma_{H_{i}}^{2}} \\
& H_{d} \sum_{i} \frac{n_{y i}}{\sigma_{H_{i}}^{2}}+\beta_{x} \sum_{i} \frac{n_{y i} n_{x i}}{\sigma_{H_{i}}^{2}}+\beta_{y} \sum_{i} \frac{n_{y i}^{2}}{\sigma_{H_{i}}^{2}} \\
& +\beta_{z} \sum_{i} \frac{n_{y i} n_{z i}}{\sigma_{H_{i}}^{2}}=\sum_{i} \frac{H_{i} n_{y i}}{\sigma_{H_{i}}^{2}} \\
& H_{d} \sum_{i} \frac{n_{z i}}{\sigma_{H_{i}}^{2}}+\beta_{x} \sum_{i} \frac{n_{z i} n_{x i}}{\sigma_{H_{i}}^{2}}+\beta_{y} \sum_{i} \frac{n_{z i} n_{y i}}{\sigma_{H_{i}}^{2}} \\
& +\beta_{z} \sum_{i} \frac{n_{z i}^{2}}{\sigma_{H_{i}}^{2}}=\sum_{i} \frac{H_{i} n_{z i}}{\sigma_{H_{i}}^{2}}
\end{aligned}
$$

which is readily solved. The covariance matrix for the original variables $Y^{a} \equiv\left(H_{d}, \beta, \ell_{d}, b_{d}\right)$ is obtained straightforwardly from the covariance matrix $\operatorname{Cov}\left(X^{c}, X^{d}\right)$ by the standard relation

$$
\operatorname{Cov}\left(Y^{a}, Y^{b}\right)=\frac{\partial Y^{a}}{\partial X^{c}} \operatorname{Cov}\left(X^{c}, X^{d}\right) \frac{\partial Y^{b}}{\partial X^{d}}
$$

where $\partial Y^{a} / \partial X^{c}$ is the relevant Jacobian.

More robust statistical results might be obtained if in place of (10) we were to fit an alternative dipole law

$$
\frac{r}{c z}=\frac{1}{H_{d}}-\frac{\beta^{\prime}}{H_{d}^{2}} \cos \phi^{\prime},
$$

since the uncertainties (B7) in $H_{i}^{-1}=r_{i} /\left(c z_{i}\right)$ are more directly related to measurement uncertainties than the uncertainties (B4) in $H_{i}$. However, while the two laws will agree if $|\beta| \ll H_{d}$, in general the relationship between (10) and (26) is not linear, so that $\beta \neq \beta^{\prime}$ and $\phi \neq \phi^{\prime}$ when $\beta / H_{d}$ can be of order $10 \%$, as is typical for the data here. Since we have plotted the variation of $H_{\alpha}$ in Figs. 57 7) rather than $H_{\alpha}^{-1}$, the only way we can expect to obtain angular agreement of the dipoles is to use (10). Furthermore, fitting (10) should give results for the dipole in angular agreement with bulk flows found in the standard peculiar velocity framework.

We have performed the analysis in the CMB and LG frames in two ways: (i) in each of the radial shells defined in Table I] and (ii) with data aggregated into inner and outer shells split at a radius $r_{o}$, as it is varied. In this way we have counterparts for both the analysis of section II and of section III A. The results, by radial shell, are given in TableVII In TableVIII we show the equivalent results for all data outside a cutoff $r>r_{o}$ as $r_{o}$ is varied.
As in the case of the spherical averages the goodness of fit of the linear relation is poor in the first few radial shells, whose radius is smaller than the typical largest voids. We have checked that using the reduced datasets of Appendix A leads to a goodness of fit close to 1.0 without substantially changing any of the conclusions.

We note that the values of $H_{d}$ are smaller than those found in Sec. II] This is a direct consequence of fitting the law (10), rather than the alternative (C6), and agrees with our observation in the case of the Gaussian window averages that when IV weightings are used a fit to (B2)(B4) rather than (B5)-(B7) gives lower mean values of $H_{\alpha}$. We have checked that if (C6) is used in place of (10) then the values of $H_{d}$ agree within $1 \sigma$ with the values of $H_{s}$ in Table $\Pi$ for those shells with $Q_{s}>0.2$ in both fits. Thus it is the relative value $\beta / H_{d}$ which is of most interest, rather than the absolute value of $\beta$, in Tables VII and VIII

\section{Appendix D: Statistical significance of results: Monte Carlo simulations}

To check that there are no systematic effects introduced by incomplete sky coverage in outer shells or the nonlinear nature of the Hubble law in inner shells, within each shell we have performed $12 \times 10^{6}$ random shuffles of the angular positions of the data points with respect to their distances and redshifts, and have recomputed the dipoles. Such a procedure will not significantly change the value of the spherically averaged Hubble constant in each shell, but would lead to a nonzero value of the dipole slope $\beta$ if the dipole in any shell originated from insufficient sky cover, for example.

The results of the Monte Carlo analysis are presented in TableIX. We find in particular that in each of $10^{6}$ shuffles per shell the weighted mean Cartesian projections of the dipole slope, $\beta_{x}, \beta_{y}$ and $\beta_{z}$ are zero to within $0.01 \sigma$ for all shells other than shell 1 which is not used in drawing statistical conclusions. For the outer 7 shells (primed or unprimed) we find $\beta_{s} / \sigma_{\beta s}<0.005$ for the random reshuffles, consistent with no dipole to high accuracy. In particular, although there are fewer data points in the outermost three shells we can be confident that any conclusions drawn from the dipole analysis are not due to unaccounted systematics.

The standard deviations $\sigma_{\beta}$ s obtained from the Monte Carlo analysis in Table IX are generally somewhat larger than the values in in Table VII, and provide a better estimate of uncertainty which accounts for systematic and nonlinear effects, particularly in the innermost shells.

In order to assess statistical confidence we have computed the $\chi^{2}$ statistic for each random shuffle, and have determined the probability, $P_{\text {ran }}$, that a random reshuffle of the angular positions of the data points yields a better linear dipole fit than that of the actual data determined in Table VII. The statistic $1-P_{\text {ran }}$ then determines the statistical confidence that we have for a non-zero dipole 


\begin{tabular}{|c|c|c|c|c|c|c|c|c|c|c|c|}
\hline Shell $s$ & 1 & 2 & 3 & 4 & 5 & 6 & 7 & 8 & 9 & 10 & 11 \\
\hline$N_{s}$ & 92 & 505 & 514 & 731 & 819 & 562 & 414 & 304 & 222 & 280 & 91 \\
\hline$r_{s}\left(h^{-1} \mathrm{Mpc}\right)$ & 2.00 & 12.50 & 25.00 & 37.50 & 50.00 & 62.50 & 75.00 & 87.50 & 100.00 & 112.50 & 156.25 \\
\hline $\bar{r}_{s}\left(h^{-1} \mathrm{Mpc}\right)$ & 5.43 & 16.33 & 30.18 & 44.48 & 55.12 & 69.24 & 81.06 & 93.75 & 105.04 & 126.27 & 182.59 \\
\hline$\left(H_{d s}\right)_{\mathrm{CMB}}$ & 95.2 & 87.2 & 94.7 & 97.5 & 96.0 & 97.9 & 97.0 & 99.7 & 100.3 & 98.4 & 95.8 \\
\hline$\left(\sigma_{H_{d s}}\right)_{\mathrm{CMB}}$ & 1.3 & 0.6 & 0.7 & 0.6 & 0.6 & 0.7 & 0.9 & 0.8 & 1.0 & 0.7 & 1.9 \\
\hline$\left(\beta_{s}\right)_{\mathrm{CMB}}$ & 95.8 & 24.4 & 14.0 & 2.6 & 4.9 & 6.8 & 3.8 & 7.4 & 5.8 & 6.4 & 0.9 \\
\hline$\left(\sigma_{\beta} s\right)_{\mathrm{CMB}}$ & 1.5 & 1.1 & 1.2 & 1.1 & 1.1 & 1.6 & 1.7 & 1.6 & 1.8 & 1.4 & 2.9 \\
\hline$\left(\ell_{d s}\right)_{\mathrm{CMB}}$ & 308.7 & 300.1 & 290.2 & 309.6 & 285.5 & 279.3 & 264.6 & 274.7 & 282.0 & 309.0 & 147.2 \\
\hline$\left(\sigma_{\ell_{d s}}\right)_{\mathrm{CMB}}$ & 1.9 & 3.0 & 6.0 & 33.9 & 13.6 & 12.7 & 25.8 & 14.7 & 21.4 & 14.5 & 389.1 \\
\hline$\left(b_{d s}\right)_{\mathrm{CMB}}$ & -5.9 & 17.3 & -5.8 & -42.0 & -3.0 & 2.2 & 12.9 & 18.8 & 11.7 & -2.8 & 48.9 \\
\hline$\left(\sigma_{b_{d s}}\right)_{\mathrm{CMB}}$ & 0.9 & 1.7 & 4.5 & 16.8 & 10.7 & 8.0 & 20.5 & 9.6 & 15.6 & 9.7 & 195.9 \\
\hline$\left(\chi^{2} / \nu\right)_{\mathrm{CMB}}$ & 15.0 & 3.1 & 1.6 & 1.0 & 0.8 & 0.7 & 0.6 & 0.6 & 0.7 & 0.9 & 0.6 \\
\hline$\left(Q_{s}\right)_{\mathrm{CMB}}$ & 0.000 & 0.000 & 0.000 & 0.332 & 1.000 & 1.000 & 1.000 & 1.000 & 1.000 & 0.817 & 0.999 \\
\hline$\left(H_{d s}\right)_{\mathrm{LG}}$ & 79.1 & 86.6 & 94.6 & 97.4 & 95.9 & 97.9 & 97.0 & 99.7 & 100.3 & 98.5 & 95.9 \\
\hline$\left(\sigma_{H_{d s}}\right)_{\mathrm{LG}}$ & 1.5 & 0.6 & 0.7 & 0.6 & 0.6 & 0.7 & 0.8 & 0.8 & 1.0 & 0.7 & 1.9 \\
\hline$\left(\beta_{s}\right)_{\mathrm{LG}}$ & 15.9 & 20.0 & 14.2 & 14.9 & 8.6 & 4.9 & 4.7 & 1.5 & 2.0 & 4.7 & 3.6 \\
\hline$\left(\sigma_{\beta s}\right)_{\mathrm{LG}}$ & 1.7 & 1.1 & 1.2 & 0.8 & 0.9 & 1.0 & 1.4 & 1.3 & 1.8 & 1.4 & 3.1 \\
\hline$\left(\ell_{d s}\right)_{\mathrm{LG}}$ & 324.6 & 52.7 & 50.3 & 87.2 & 85.3 & 86.3 & 107.0 & 265.4 & 353.4 & 348.1 & 111.3 \\
\hline$\left(\sigma_{\ell_{d s}}\right)_{\mathrm{LG}}$ & 12.7 & 3.9 & 8.3 & 4.9 & 10.1 & 32.9 & 27.9 & 115.6 & 217.1 & 24.2 & 76.0 \\
\hline$\left(b_{d s}\right)_{\mathrm{LG}}$ & -30.5 & -34.8 & -55.4 & -36.3 & -44.2 & -58.7 & -42.5 & -51.0 & -76.2 & -38.1 & -17.8 \\
\hline$\left(\sigma_{b_{d s}}\right)_{\mathrm{LG}}$ & 6.4 & 2.2 & 5.5 & 4.3 & 8.0 & 18.5 & 20.7 & 59.3 & 57.3 & 14.3 & 36.5 \\
\hline$\left(\chi^{2} / \nu\right)_{\mathrm{LG}}$ & 7.8 & 3.6 & 1.6 & 1.1 & 0.8 & 0.7 & 0.6 & 0.6 & 0.7 & 0.9 & 0.6 \\
\hline$\left(Q_{s}\right)_{\mathrm{LG}}$ & 0.000 & 0.000 & 0.000 & 0.158 & 1.000 & 1.000 & 1.000 & 1.000 & 1.000 & 0.882 & 1.000 \\
\hline Shell $s$ & $1^{\prime}$ & $2^{\prime}$ & $3^{\prime}$ & $4^{\prime}$ & $5^{\prime}$ & $6^{\prime}$ & 7 & $8^{\prime}$ & 9' & $10^{\prime}$ & 11 \\
\hline$N_{s}$ & 321 & 513 & 553 & 893 & 681 & 485 & 343 & 273 & 164 & 206 & 91 \\
\hline$r_{s}\left(h^{-1} \mathrm{Mpc}\right)$ & 6.25 & 18.75 & 31.25 & 43.75 & 56.25 & 68.75 & 81.25 & 93.75 & 106.25 & 118.75 & 156.25 \\
\hline $\bar{r}_{s}\left(h^{-1} \mathrm{Mpc}\right)$ & 12.26 & 23.46 & 37.61 & 49.11 & 61.74 & 73.92 & 87.15 & 99.12 & 111.95 & 131.49 & 182.59 \\
\hline$\left(H_{d s}\right)_{\mathrm{CMB}}$ & 87.5 & 90.4 & 93.8 & 97.5 & 96.1 & 98.2 & 98.5 & 100.5 & 97.2 & 99.3 & 95.8 \\
\hline$\left(\sigma_{H_{d s}}\right)_{\mathrm{CMB}}$ & 0.7 & 0.6 & 0.7 & 0.6 & 0.7 & 0.7 & 0.9 & 0.9 & 1.0 & 0.9 & 1.9 \\
\hline$\left(\beta_{s}\right)_{\mathrm{CMB}}$ & 39.5 & 16.2 & 8.5 & 3.0 & 5.8 & 5.2 & 5.0 & 8.9 & 6.7 & 5.4 & 0.9 \\
\hline$\left(\sigma_{\beta s}\right)_{\mathrm{CMB}}$ & 1.2 & 1.1 & 1.2 & 1.0 & 1.3 & 1.6 & 1.6 & 1.7 & 2.1 & 1.5 & 2.9 \\
\hline$\left(\ell_{d s}\right)_{\mathrm{CMB}}$ & 294.9 & 296.1 & 307.3 & 273.9 & 276.2 & 270.1 & 289.3 & 279.7 & 314.8 & 308.8 & 147.2 \\
\hline$\left(\sigma_{\ell_{d s}}\right)_{\mathrm{CMB}}$ & 2.6 & 4.6 & 10.1 & 20.2 & 12.8 & 17.8 & 21.0 & 12.0 & 17.0 & 20.7 & 389.1 \\
\hline$\left(b_{d s}\right)_{\mathrm{CMB}}$ & 17.9 & 15.2 & -13.4 & -16.6 & 14.9 & -0.1 & 26.8 & 7.0 & 4.3 & 2.1 & 48.9 \\
\hline$\left(\sigma_{b_{d s}}\right)_{\mathrm{CMB}}$ & 1.2 & 3.5 & 7.0 & 15.4 & 11.4 & 10.2 & 16.9 & 8.4 & 13.9 & 14.2 & 195.9 \\
\hline$\left(\chi^{2} / \nu\right)_{\mathrm{CMB}}$ & 3.9 & 2.1 & 1.4 & 0.9 & 0.8 & 0.6 & 0.6 & 0.7 & 1.0 & 0.6 & 0.6 \\
\hline$\left(Q_{s}\right)_{\mathrm{CMB}}$ & 0.000 & 0.000 & 0.000 & 0.997 & 1.000 & 1.000 & 1.000 & 1.000 & 0.333 & 1.000 & 0.999 \\
\hline$\left(H_{d s}\right)_{\mathrm{LG}}$ & 88.2 & 89.4 & 94.2 & 97.4 & 96.2 & 98.2 & 98.4 & 100.5 & 97.3 & 99.4 & 95.9 \\
\hline$\left(\sigma_{H_{d s}}\right)_{\mathrm{LG}}$ & 0.8 & 0.7 & 0.7 & 0.5 & 0.7 & 0.7 & 0.9 & 0.9 & 1.0 & 0.9 & 1.9 \\
\hline${ }^{\left(\beta_{s}\right)} \mathrm{LG}$ & 20.9 & 15.9 & 15.1 & 11.8 & 5.4 & 5.1 & 2.8 & 3.7 & 5.0 & 3.6 & 3.6 \\
\hline$\left(\sigma_{\beta s}\right)_{\mathrm{LG}}$ & 1.6 & 1.1 & 1.1 & 0.8 & 1.1 & 1.0 & 1.5 & 1.6 & 2.2 & 1.6 & 3.1 \\
\hline$\left(\ell_{d s}\right)_{\mathrm{LG}}$ & 42.9 & 51.9 & 64.0 & 94.5 & 94.1 & 103.7 & 70.4 & 286.4 & 0.0 & 360.0 & 111.3 \\
\hline$\left(\sigma_{\ell_{d s}}\right)_{\mathrm{LG}}$ & 4.5 & 4.8 & 6.0 & 6.3 & 17.2 & 33.9 & 43.3 & 35.4 & 27.1 & 33.9 & 76.0 \\
\hline$\left(b_{d s}\right)_{\mathrm{LG}}$ & -35.5 & -31.8 & -43.4 & -38.0 & -38.8 & -57.8 & -33.8 & -37.0 & -29.5 & -36.7 & -17.8 \\
\hline$\left(\sigma_{b_{d s}}\right)_{\mathrm{LG}}$ & 2.7 & 3.7 & 5.1 & 5.1 & 14.9 & 17.1 & 31.4 & 23.1 & 16.0 & 24.4 & 36.5 \\
\hline$\left(\chi^{2} / \nu\right) \mathrm{LG}$ & 4.3 & 2.7 & 1.4 & 0.9 & 0.8 & 0.6 & 0.6 & 0.7 & 1.0 & 0.6 & 0.6 \\
\hline$\left(Q_{s}\right)_{\mathrm{LG}}$ & 0.000 & 0.000 & 0.000 & 0.988 & 1.000 & 1.000 & 1.000 & 1.000 & 0.404 & 1.000 & 1.000 \\
\hline
\end{tabular}

TABLE VII: Least squares fit of dipole Hubble law (10) in radial shells in CMB and LG frames, for the same two choices of shells given in Table [. We tabulate $N_{s}, r_{s}, \bar{r}_{s}$; the best fit dipole Hubble constant, $H_{d s}$ (units $h \mathrm{~km} \mathrm{~s}^{-1} \mathrm{Mpc}^{-1}$ ), dipole slope $\beta_{s}$ (units $h \mathrm{~km} \mathrm{~s}^{-1} \mathrm{Mpc}^{-1}$ ), the galactic longitude $\ell_{d s}$ and latitude $b_{d s}$ of the dipole apex, and their respective standard deviations $\sigma_{H_{d s}}, \sigma_{\beta s}, \sigma_{b_{d s}}$ and $\sigma_{\ell_{d s}}$. We also tabulate the reduced $\chi^{2}$ (for $\nu=N_{s}-4$ ) and goodness of fit probability $Q_{s}$ in each case.

in each shell. For example, in the outermost control shell $98.3 \%$ of random angular reshuffles in the CMB frame and $69.1 \%$ of reshuffles in the LG frame give a better fit than the data, meaning that there is no evidence for a dipole in that shell in either frame, consistent with the angular uncertainties already noted. Since the same conclusion applies to both frames, there are insufficient data points to establish the existence of a dipole, and much more data beyond $150 h^{-1} \mathrm{Mpc}$ is required to determine whether a dipole is genuinely absent. 


\begin{tabular}{|c|c|c|c|c|c|c|c|c|c|c|c|}
\hline \multicolumn{12}{|c|}{ CMB frame } \\
\hline$r_{o}$ & $N$ & $H_{d}$ & $\sigma_{H_{d}}$ & $\beta$ & $\sigma_{\beta}$ & $\ell_{d}$ & $\sigma_{\ell_{d}}$ & $b_{d}$ & $\sigma_{b_{d}}$ & $\chi^{2} / \nu$ & $Q$ \\
\hline 15 & 4358 & 96.0 & 0.2 & 6.1 & 0.4 & 300.2 & 4.3 & 6.1 & 3.1 & 1.18 & 0.000 \\
\hline 20 & 4158 & 97.1 & 0.2 & 5.8 & 0.4 & 289.6 & 4.7 & 1.0 & 3.5 & 1.04 & 0.032 \\
\hline 25 & 3937 & 97.6 & 0.2 & 5.5 & 0.5 & 290.4 & 5.2 & 0.7 & 3.8 & 0.92 & 1.000 \\
\hline 30 & 3742 & 97.6 & 0.2 & 4.8 & 0.5 & 291.7 & 6.0 & 2.9 & 4.4 & 0.87 & 1.000 \\
\hline 35 & 3538 & 97.8 & 0.3 & 4.4 & 0.5 & 292.0 & 6.7 & 3.3 & 4.9 & 0.83 & 1.000 \\
\hline 40 & 3308 & 97.8 & 0.3 & 4.1 & 0.5 & 288.7 & 7.4 & 4.8 & 5.5 & 0.78 & 1.000 \\
\hline 45 & 3055 & 98.1 & 0.3 & 4.5 & 0.5 & 283.4 & 7.1 & 7.8 & 5.3 & 0.76 & 1.000 \\
\hline 50 & 2692 & 97.9 & 0.3 & 5.4 & 0.6 & 289.0 & 6.5 & 8.3 & 4.6 & 0.73 & 1.000 \\
\hline 55 & 2328 & 98.1 & 0.3 & 5.6 & 0.6 & 286.2 & 6.6 & 9.2 & 4.7 & 0.71 & 1.000 \\
\hline 60 & 2008 & 98.3 & 0.3 & 5.3 & 0.7 & 291.5 & 7.4 & 7.3 & 5.2 & 0.71 & 1.000 \\
\hline 65 & 1741 & 98.5 & 0.3 & 5.5 & 0.7 & 289.0 & 7.8 & 7.8 & 5.3 & 0.69 & 1.000 \\
\hline 70 & 1520 & 98.6 & 0.4 & 5.4 & 0.7 & 289.1 & 8.1 & 6.8 & 5.5 & 0.68 & 1.000 \\
\hline 75 & 1311 & 98.6 & 0.4 & 5.3 & 0.8 & 289.8 & 9.0 & 11.3 & 6.5 & 0.69 & 1.000 \\
\hline 80 & 1124 & 98.7 & 0.4 & 5.6 & 0.8 & 291.8 & 8.9 & 9.2 & 6.4 & 0.72 & 1.000 \\
\hline 85 & 965 & 99.0 & 0.4 & 5.8 & 0.8 & 291.1 & 9.2 & 7.8 & 6.5 & 0.72 & 1.000 \\
\hline 90 & 833 & 99.1 & 0.5 & 5.7 & 0.9 & 292.9 & 9.9 & 8.1 & 7.1 & 0.74 & 1.000 \\
\hline 95 & 704 & 99.1 & 0.5 & 6.0 & 0.9 & 297.8 & 10.1 & 3.3 & 7.0 & 0.75 & 1.000 \\
\hline 100 & 593 & 98.9 & 0.5 & 5.5 & 1.0 & 303.3 & 12.0 & 4.6 & 8.4 & 0.79 & 1.000 \\
\hline 105 & 480 & 98.2 & 0.6 & 5.7 & 1.1 & 308.8 & 13.0 & 6.6 & 9.3 & 0.79 & 1.000 \\
\hline 110 & 401 & 98.3 & 0.6 & 5.1 & 1.2 & 311.0 & 16.3 & 1.6 & 10.7 & 0.80 & 0.998 \\
\hline 115 & 343 & 98.3 & 0.7 & 5.1 & 1.3 & 312.4 & 18.0 & 3.5 & 11.9 & 0.86 & 0.975 \\
\hline 120 & 287 & 98.5 & 0.8 & 4.6 & 1.4 & 319.1 & 22.2 & 7.0 & 14.5 & 0.62 & 1.000 \\
\hline \multicolumn{12}{|c|}{ LG frame } \\
\hline$r_{o}$ & $N$ & $H_{d}$ & $\sigma_{H_{d}}$ & $\beta$ & $\sigma_{\beta}$ & $\ell_{d}$ & $\sigma_{\ell_{d}}$ & $b_{d}$ & $\sigma_{b_{d}}$ & $\chi^{2} / \nu$ & $Q$ \\
\hline 15 & 4358 & 94.7 & 0.2 & 11.4 & 0.4 & 68.3 & 2.7 & -38.0 & 2.0 & 1.39 & 0.000 \\
\hline 20 & 4158 & 96.1 & 0.2 & 9.4 & 0.4 & 78.0 & 3.4 & -36.2 & 2.6 & 1.18 & 0.000 \\
\hline 25 & 3937 & 97.1 & 0.2 & 8.0 & 0.4 & 80.8 & 4.4 & -40.2 & 3.2 & 0.97 & 0.906 \\
\hline 30 & 3742 & 97.2 & 0.2 & 7.7 & 0.4 & 83.1 & 4.6 & -38.2 & 3.4 & 0.93 & 0.999 \\
\hline 35 & 3538 & 97.5 & 0.3 & 7.4 & 0.4 & 82.8 & 4.9 & -38.1 & 3.7 & 0.89 & 1.000 \\
\hline 40 & 3308 & 97.7 & 0.3 & 6.9 & 0.4 & 85.6 & 5.4 & -38.6 & 4.0 & 0.84 & 1.000 \\
\hline 45 & 3055 & 98.1 & 0.3 & 5.6 & 0.4 & 89.4 & 7.5 & -42.7 & 5.3 & 0.79 & 1.000 \\
\hline 50 & 2692 & 97.8 & 0.3 & 4.3 & 0.5 & 68.4 & 12.6 & -52.2 & 7.6 & 0.75 & 1.000 \\
\hline 55 & 2328 & 98.0 & 0.3 & 3.5 & 0.5 & 55.1 & 21.4 & -60.7 & 10.2 & 0.71 & 1.000 \\
\hline 60 & 2008 & 98.2 & 0.3 & 3.6 & 0.5 & 45.9 & 20.1 & -57.8 & 10.1 & 0.72 & 1.000 \\
\hline 65 & 1741 & 98.4 & 0.3 & 3.3 & 0.6 & 31.3 & 26.5 & -61.9 & 11.7 & 0.69 & 1.000 \\
\hline 70 & 1520 & 98.5 & 0.4 & 3.2 & 0.6 & 20.6 & 29.4 & -62.9 & 12.6 & 0.68 & 1.000 \\
\hline 75 & 1311 & 98.6 & 0.4 & 2.6 & 0.7 & 17.0 & 31.2 & -56.6 & 15.7 & 0.68 & 1.000 \\
\hline 80 & 1124 & 98.7 & 0.4 & 2.8 & 0.8 & 0.2 & 28.3 & -53.3 & 15.1 & 0.71 & 1.000 \\
\hline 85 & 965 & 99.0 & 0.4 & 2.7 & 0.8 & 345.6 & 29.4 & -52.5 & 15.8 & 0.71 & 1.000 \\
\hline 90 & 833 & 99.1 & 0.5 & 2.7 & 0.9 & 347.1 & 28.1 & -48.2 & 16.2 & 0.73 & 1.000 \\
\hline 95 & 704 & 99.0 & 0.5 & 3.3 & 1.0 & 343.9 & 23.3 & -44.8 & 13.5 & 0.74 & 1.000 \\
\hline 100 & 593 & 98.8 & 0.5 & 3.4 & 1.1 & 355.9 & 23.8 & -41.2 & 14.6 & 0.78 & 1.000 \\
\hline 105 & 480 & 98.2 & 0.6 & 3.6 & 1.2 & 357.9 & 21.8 & -32.9 & 14.7 & 0.77 & 1.000 \\
\hline 110 & 401 & 98.3 & 0.6 & 3.8 & 1.2 & 3.7 & 25.4 & -40.1 & 17.2 & 0.79 & 0.999 \\
\hline 115 & 343 & 98.4 & 0.7 & 3.7 & 1.4 & 4.3 & 25.6 & -36.1 & 19.2 & 0.83 & 0.989 \\
\hline 120 & 287 & 98.6 & 0.8 & 3.5 & 1.6 & 18.1 & 25.5 & -29.9 & 22.3 & 0.61 & 1.000 \\
\hline
\end{tabular}

TABLE VIII: Least squares fit of dipole Hubble law (10) using data outside a radius $r>r_{o}$, as $r_{o}$ is varied, in both CMB and LG frames. We tabulate $r_{o}, N\left(r>r_{o}\right.$ ), the best fit dipole Hubble constant, $H_{d}$ (units $h \mathrm{~km} \mathrm{~s}^{-1} \mathrm{Mpc}^{-1}$ ), dipole slope $\beta$ (units $h \mathrm{~km} \mathrm{~s}^{-1} \mathrm{Mpc}^{-1}$ ), the galactic longitude $\ell_{d}$ and latitude $b_{d}$ of the dipole apex, and their respective standard deviations $\sigma_{H_{d}}$, $\sigma_{\beta}, \sigma_{b_{d}}$ and $\sigma_{\ell_{d}}$. We also tabulate the reduced $\chi^{2}$ (for $\nu=N-4$ ) and goodness of fit probability $Q$ in each case.

The values of $P_{\text {ran }}$ lead to a robust interpretation of Fig. 9. Tables VII and IX show that in the unprimed shells ${ }^{24}$ there is a significant dipole at the $90 \%$ confidence

24 The conclusions drawn from the primed shells are similar, with the proviso that in shell 9' the CMB frame dipole is only found at the $88.5 \%$ confidence level. Shell 9' has only 164 points, and level in all shells up to $s=10$ apart from shell 4 , with

the goodness of fit for a dipole Hubble law is only 0.132 and 0.404 in the CMB and LG frames, as compared to 0.992 and 0.996 respectively for the monopole Hubble law. Evidently the number of points in the outer shells needs to be of the order of at least 200 in order to make statements about the dipoles at the $90 \%$ confidence level in at least one frame. Fortunately this is true of the unprimed shells. 


\begin{tabular}{|c|c|c|c|c|c|c|c|c|c|c|c|}
\hline Shell $s$ & 1 & 2 & 3 & 4 & 5 & 6 & 7 & 8 & 9 & 10 & 11 \\
\hline$\left(\beta_{x s}\right)_{\mathrm{CMB}}$ & -0.561 & 0.002 & 0.004 & 0.002 & -0.002 & 0.000 & -0.001 & -0.006 & -0.006 & 0.001 & 0.004 \\
\hline$\left(\sigma_{\beta x s}\right)_{\mathrm{CMB}}$ & 55.8 & 4.3 & 3.1 & 1.5 & 1.2 & 1.3 & 1.6 & 1.9 & 2.3 & 2.1 & 3.5 \\
\hline$\left(\beta_{y s}\right)_{\mathrm{CMB}}$ & 2.533 & -0.002 & -0.011 & 0.000 & 0.001 & 0.003 & 0.002 & 0.007 & 0.002 & -0.004 & -0.017 \\
\hline$\left(\sigma_{\beta y} s\right)_{\mathrm{CMB}}$ & 46.4 & 4.1 & 2.8 & 1.5 & 1.3 & 1.3 & 1.6 & 1.7 & 2.1 & 2.1 & 2.7 \\
\hline$\left(\beta_{z s}\right)_{\mathrm{CMB}}$ & -6.212 & 0.000 & 0.006 & 0.000 & 0.001 & 0.002 & 0.000 & -0.001 & -0.004 & -0.001 & 0.002 \\
\hline$\left(\sigma_{\beta z s}\right)_{\mathrm{CMB}}$ & 36.3 & 3.2 & 2.4 & 1.3 & 1.0 & 0.9 & 1.3 & 1.5 & 1.7 & 1.5 & 2.2 \\
\hline$\left(\beta_{s}\right)_{\mathrm{CMB}}$ & 6.732 & 0.003 & 0.013 & 0.002 & 0.002 & 0.003 & 0.002 & 0.010 & 0.008 & 0.004 & 0.018 \\
\hline$\left(\sigma_{\beta s}\right)_{\mathrm{CMB}}$ & 55.6 & 6.3 & 4.4 & 1.7 & 1.8 & 1.6 & 2.2 & 2.7 & 3.2 & 2.5 & 3.6 \\
\hline$\left(P_{\text {ran }}\right)_{\mathrm{CMB}}$ & 0.000 & 0.000 & 0.000 & 0.435 & 0.001 & 0.000 & 0.093 & 0.000 & 0.034 & 0.003 & 0.983 \\
\hline$\left(\beta_{x s}\right)_{\mathrm{LG}}$ & -0.012 & -0.028 & 0.000 & 0.018 & -0.001 & -0.003 & -0.001 & -0.003 & -0.003 & 0.001 & 0.001 \\
\hline$\left(\sigma_{\beta_{x} s}\right)_{\mathrm{LG}}$ & 13.7 & 4.2 & 2.7 & 3.1 & 1.5 & 1.3 & 1.7 & 1.7 & 1.9 & 2.0 & 3.7 \\
\hline$\left(\beta_{y s}\right)_{\mathrm{LG}}$ & 0.322 & 0.053 & -0.001 & 0.009 & 0.000 & -0.003 & 0.002 & 0.003 & 0.001 & -0.004 & -0.008 \\
\hline$\left(\sigma_{\beta y s}\right)_{\mathrm{LG}}$ & 11.4 & 4.1 & 2.4 & 3.2 & 1.5 & 1.4 & 1.7 & 1.6 & 1.8 & 2.0 & 2.9 \\
\hline$\left(\beta_{z s}\right)_{\mathrm{LG}}$ & -0.478 & -0.019 & -0.001 & 0.002 & 0.000 & -0.007 & -0.001 & -0.001 & -0.002 & -0.001 & 0.000 \\
\hline$\left(\sigma_{\beta_{z} s}\right)_{\mathrm{LG}}$ & 8.4 & 3.2 & 2.0 & 2.7 & 1.2 & 1.0 & 1.3 & 1.3 & 1.4 & 1.5 & 2.4 \\
\hline$\left(\beta_{s}\right)_{\mathrm{LG}}$ & 0.576 & 0.064 & 0.001 & 0.020 & 0.001 & 0.008 & 0.002 & 0.004 & 0.003 & 0.004 & 0.008 \\
\hline$\left(\sigma_{\beta s}\right)_{\mathrm{LG}}$ & 13.6 & 6.3 & 3.3 & 4.5 & 1.9 & 1.8 & 2.6 & 2.6 & 2.8 & 2.5 & 3.2 \\
\hline$\left(P_{\text {ran }}\right)_{\mathrm{LG}}$ & 0.000 & 0.000 & 0.000 & 0.000 & 0.000 & 0.000 & 0.002 & 0.718 & 0.624 & 0.040 & 0.691 \\
\hline Shell $s$ & $1 '$ & 2 ' & $3^{\prime}$ & $4^{\prime}$ & 5 & $6^{\prime}$ & 7 & $8^{\prime}$ & 9' & 10 ' & 11 \\
\hline$\left(\beta_{x s}\right)_{\mathrm{CMB}}$ & -0.003 & -0.006 & 0.001 & 0.001 & 0.002 & -0.001 & -0.004 & -0.003 & -0.005 & 0.002 & 0.002 \\
\hline$\left(\sigma_{\beta_{x} s}\right)_{\mathrm{CMB}}$ & 8.0 & 3.3 & 2.5 & 1.1 & 1.5 & 1.4 & 1.6 & 2.1 & 3.2 & 2.0 & 3.5 \\
\hline$\left(\beta_{y s}\right)_{\mathrm{CMB}}$ & 0.012 & 0.016 & 0.002 & 0.000 & 0.001 & 0.002 & 0.000 & 0.010 & 0.001 & -0.006 & -0.015 \\
\hline$\left(\sigma_{\beta y} s\right)_{\mathrm{CMB}}$ & 7.2 & 3.1 & 2.4 & 1.2 & 1.6 & 1.3 & 1.7 & 1.9 & 2.9 & 2.1 & 2.7 \\
\hline$\left(\beta_{z s}\right)_{\mathrm{CMB}}$ & 0.000 & -0.008 & -0.004 & 0.001 & 0.000 & -0.001 & -0.003 & -0.003 & -0.004 & 0.001 & 0.000 \\
\hline$\left(\sigma_{\beta z_{s}}\right)_{\mathrm{CMB}}$ & 4.9 & 2.7 & 1.9 & 1.0 & 1.2 & 1.0 & 1.3 & 1.6 & 2.3 & 1.5 & 2.2 \\
\hline$\left(\beta_{s}\right)_{\mathrm{CMB}}$ & 0.012 & 0.019 & 0.004 & 0.001 & 0.002 & 0.002 & 0.005 & 0.011 & 0.006 & 0.006 & 0.015 \\
\hline$\left(\sigma_{\beta s}\right)_{\mathrm{CMB}}$ & 8.9 & 4.8 & 3.1 & 1.5 & 2.2 & 2.0 & 2.2 & 2.7 & 4.5 & 2.8 & 3.2 \\
\hline$\left(P_{\mathrm{ran}}\right)_{\mathrm{CMB}}$ & 0.000 & 0.000 & 0.000 & 0.112 & 0.000 & 0.003 & 0.008 & 0.000 & 0.115 & 0.008 & 0.983 \\
\hline$\left(\beta_{x s}\right)_{\mathrm{LG}}$ & -0.019 & -0.016 & 0.003 & 0.002 & -0.002 & 0.001 & -0.002 & -0.002 & -0.006 & 0.001 & 0.000 \\
\hline$\left(\sigma_{\beta x s}\right)_{\mathrm{LG}}$ & 5.2 & 4.4 & 3.4 & 1.9 & 1.5 & 1.5 & 1.7 & 1.8 & 3.0 & 1.8 & 3.7 \\
\hline$\left(\beta_{y s}\right)_{\mathrm{LG}}$ & 0.052 & 0.040 & 0.026 & 0.001 & -0.001 & -0.005 & -0.001 & 0.008 & -0.001 & -0.004 & -0.005 \\
\hline$\left(\sigma_{\beta y} s\right)_{\mathrm{LG}}$ & 4.7 & 4.2 & 3.3 & 2.0 & 1.6 & 1.5 & 1.7 & 1.6 & 2.7 & 1.9 & 2.9 \\
\hline$\left(\beta_{z s}\right)_{\mathrm{LG}}$ & -0.041 & -0.015 & -0.016 & 0.005 & -0.002 & 0.000 & -0.005 & -0.002 & -0.006 & 0.001 & -0.001 \\
\hline$\left(\sigma_{\beta_{z s}}\right)_{\mathrm{LG}}$ & 3.2 & 3.6 & 2.7 & 1.7 & 1.2 & 1.1 & 1.3 & 1.4 & 2.1 & 1.4 & 2.4 \\
\hline$\left(\beta_{s}\right)_{\mathrm{LG}}$ & 0.069 & 0.046 & 0.030 & 0.006 & 0.003 & 0.006 & 0.005 & 0.008 & 0.009 & 0.004 & 0.005 \\
\hline$\left(\sigma_{\beta s}\right)_{\mathrm{LG}}$ & 6.8 & 6.5 & 4.5 & 2.7 & 2.3 & 1.8 & 2.1 & 2.3 & 3.8 & 2.5 & 3.3 \\
\hline$\left(P_{\text {ran }}\right)_{\mathrm{LG}}$ & 0.000 & 0.000 & 0.000 & 0.000 & 0.000 & 0.000 & 0.255 & 0.080 & 0.422 & 0.146 & 0.691 \\
\hline
\end{tabular}

TABLE IX: Monte Carlo analysis of randomized angular variations by radial shell in CMB and LG frames. The analysis of Table VII is reperformed with random shuffling of angular coordinates of the data points in radial shells in each rest frame. For each shell, and each rest frame, we tabulate the weighted mean Cartesian projections $\beta_{x}, \beta_{y}, \beta_{z}$ (defined in Appendix C), the combined dipole magnitude, $\beta$, and their weighted standard deviations. We also tabulate the probability, $P_{\text {ran }}$, that a random shuffle in each shell produced a better fit to the linear dipole law (10) than the actual data. The results are based on $10^{6}$ shuffles in each shell, and 12 independent runs.

$\bar{r}_{4}=44.5 h^{-1} \mathrm{Mpc}$, where as has been noted the CMB frame also provides a better fit to a monopole Hubble law. In shell $4,43.5 \%$ of random angular shuffles in the CMB frame produce a better dipole fit, meaning that the dipole is found with only $56.5 \%$ confidence. By contrast, in the LG frame the dipole is found at a confidence level of more than $99.999 \%$ in shell 4 . The difference is therefore a frame effect.

In the unprimed shells in the LG frame there is a significant dipole at the $90 \%$ confidence level in all shells $s \leq 7$ and also in shell 10. In shells 8 and 9 the probability of a random angular shuffle giving a better fit than the data is greater than $50 \%$, consistent with the values of $\beta$ close to zero shown in Fig. 9 By contrast a dipole is found in the CMB frame in shells 8 and 9 respectively at the levels of $99.98 \%$ and $96.6 \%$ confidence. Thus we can be confident that the absence of the dipole in the LG frame in shells 8 and 9 is a genuine frame effect, rather than being due to small number statistics.

In shell 10, whose inner boundary $r_{10}=112.5 h^{-1} \mathrm{Mpc}$ is close to the $\mathrm{BAO}$ scale, there is a dipole at the level of $96 \%$ confidence in the LG frame and $99.7 \%$ in the CMB frame. Since shells 8 and 9 lack a significant dipole in the LG frame, it would appear that it is the feature 
in this shell that is driving the appearance of the residual LG frame dipole for the largest radii shown in the smoothed value of $\beta$ shown in Fig. 10 Furthermore, since there is only a LG frame dipole at the $85.4 \%$ confidence level in shell 10', whose inner boundary is at $r_{s}=118.75 h^{-1} \mathrm{Mpc}$, it would appear that the data in the closer portions of shell 10 contains the relevant structures. A great deal more data should be added to the analysis before we draw any firm conclusions about features in this shell, however.
[1] A. Kogut, C. Lineweaver, G.F. Smoot, et al., Astrophys. J. 419, 1 (1993).

[2] D.J. Fixsen, E.S. Cheng, J.M. Gales, J.C. Mather, R.A. Shafer, and E.L. Wright, Astrophys. J. 473, 576 (1996).

[3] D.W. Hogg, D.J. Eisenstein, M.R. Blanton, N.A. Bahcall, J. Brinkmann, J.E. Gunn, and D.P. Schneider, Astrophys. J. 624, 54 (2005).

[4] F. Sylos Labini, N.L. Vasilyev, L. Pietronero, and Y.V. Baryshev, Europhys. Lett. 86, 49001 (2009).

[5] M. Scrimgeour, T. Davis, C. Blake, et al., Mon. Not. R. Astr. Soc. 425, 116 (2012).

[6] R.G. Clowes, K.A. Harris, S. Raghunathan, L.E. Campusano, I.K. Soechting, and M.J. Graham, Mon. Not. R. Astr. Soc. 429, 2910 (2013).

[7] M.J. Hudson, R.J. Smith, J.R. Lucey, and E. Branchini, Mon. Not. R. Astr. Soc. 352, 61 (2004).

[8] D.D. Kocevski and H. Ebeling, Astrophys. J. 645, 1043 (2006).

[9] C.M. Springob, K.L. Masters, M.P. Haynes, R. Giovanelli, and C. Marinoni, Astrophys. J. Suppl. 172, 599 (2007).

[10] R.B. Tully, E.J. Shaya, I.D. Karachentsev, H.M. Courtois, D.D. Kocevski, L. Rizzi, and A. Peel, Astrophys. J. 676, 184 (2008).

[11] I.D. Karachentsev, O.G. Kashibadze, D.I. Makarov, and R.B. Tully, Mon. Not. R. Astr. Soc. 393, 1265 (2009).

[12] G. Lavaux, R.B. Tully, R. Mohayaee, and S. Colombi, Astrophys. J. 709, 483 (2010).

[13] I. Antoniou and L. Perivolaropoulos, J. Cosmol. Astropart. Phys. 12 (2010) 012.

[14] J. Colin, R. Mohayaee, S. Sarkar, and A. Shafieloo, Mon. Not. R. Astr. Soc. 414, 264 (2011).

[15] D.C. Dai, W.H. Kinney, and D. Stojkovic, J. Cosmol. Astropart. Phys. 04 (2011) 015.

[16] A. Weyant, M. Wood-Vasey, L. Wasserman, and P. Freeman, Astrophys. J. 732, 65 (2011).

[17] I. Iwata and P. Chamaraux, Astron. Astrophys. 531, A87 (2011).

[18] R. Watkins, H.A. Feldman, and M.J. Hudson, Mon. Not. R. Astr. Soc. 392, 743 (2009).

[19] H.A. Feldman, R. Watkins, and M.J. Hudson, Mon. Not. R. Astr. Soc. 407, 2328 (2010).

[20] A. Kashlinsky, F. Atrio-Barandela, D. Kocevski, and H. Ebeling, Astrophys. J. 686, L49 (2008).

[21] A. Kashlinsky, F. Atrio-Barandela, H. Ebeling, A. Edge, and D. Kocevski, Astrophys. J. 712, L81 (2010).

[22] A. Nusser and M. Davis, Astrophys. J. 736, 93 (2011).

[23] Y.Z. Ma and D. Scott, Mon. Not. R. Astr. Soc. 428, 2017 (2013).

[24] S.J. Turnbull, M.J. Hudson, H.A. Feldman, M. Hicken, R.P. Kirshner, and R. Watkins, Mon. Not. R. Astr. Soc. 420, 447 (2012).

[25] M.L. McClure and C.C. Dyer, New Astron. 12, 533
(2007).

[26] N. Li and D.J. Schwarz, Phys. Rev. D 78, 083531 (2008).

[27] D.L. Wiltshire, Class. Quantum Grav. 28, 164006 (2011).

[28] M. Tegmark, A. de Oliveira-Costa, and A.J.S. Hamilton, Phys. Rev. D 68, 123523 (2003).

[29] K. Land and J. Magueijo, Phys. Rev. Lett. 95, 071301 (2005).

[30] H.K. Eriksen, A.J. Banday, K.M. Gorski, F.K. Hansen, and P.B. Lilje, Astrophys. J. 660, L81 (2007).

[31] J. Hoftuft, H.K. Eriksen, A.J. Banday, K.M. Gorski, F.K. Hansen, and P.B. Lilje, Astrophys. J. 699, 985 (2009).

[32] J. Kim and P. Naselsky, Astrophys. J. 714, L265 (2010).

[33] P.A.R. Ade, et al., arXiv:1303.5083 (2013).

[34] D.L. Wiltshire, New J. Phys. 9, 377 (2007).

[35] D.L. Wiltshire, Phys. Rev. Lett. 99, 251101 (2007).

[36] D.L. Wiltshire, Phys. Rev. D 78, 084032 (2008).

[37] D.L. Wiltshire, Phys. Rev. D 80, 123512 (2009).

[38] F. Hoyle and M.S. Vogeley, Astrophys. J. 566, 641 (2002).

[39] F. Hoyle and M.S. Vogeley, Astrophys. J. 607, 751 (2004).

[40] D.C. Pan, M.S. Vogeley, F. Hoyle, Y.Y. Choi, and C. Park, Mon. Not. R. Astr. Soc. 421, 926 (2012).

[41] W.L. Freedman, B.F. Madore, B.K. Gibson, et al., Astrophys. J. 553, 47 (2001).

[42] T.R. Lauer and M. Postman, Astrophys. J. 425, 418 (1994).

[43] W.H. Press, B.P. Flannery, S.A. Teukolsky, and W.T. Vetterling, Numerical Recipes, (Cambridge University Press, 1986).

[44] C.L. Bennett, M. Halpern, G. Hinshaw, et al., Astrophys. J. Suppl. 148, 1 (2003).

[45] R.E. Kass and A.E. Raftery, J. Am. Statist. Assoc. 90, 773 (1995).

[46] R. Trotta, Mon. Not. R. Astr. Soc. 378, 72 (2007).

[47] K.M. Gorski, E. Hivon, A.J. Banday, B.D. Wandelt, F.K. Hansen, M. Reinecke, and M. Bartelman, Astrophys. J. 622, 759 (2005).

[48] A. Sandage, Astrophys. J. 307, 1 (1986).

[49] A. Kashlinsky, F. Atrio-Barandela, D. Kocevski, and H. Ebeling, Astrophys. J. 691, 1479 (2009).

[50] J.K. Webb, J.A. King, M.T. Murphy, V.V. Flambaum, R.F. Carswell, and M.B. Bainbridge, Phys. Rev. Lett. 107, 191101 (2011).

[51] J.A. King, J.K. Webb, M.T. Murphy, et al.Mon. Not. R. Astr. Soc. 422, 3370 (2012).

[52] A. Mariano and L. Perivolaropoulos, Phys. Rev. D 86, 083517 (2012).

[53] A. Mariano and L. Perivolaropoulos, Phys. Rev. D 87, 043511 (2013).

[54] P. Erdoğdu, O. Lahav, J.P. Huchra, et al., Mon. Not. 
R. Astr. Soc. 373, 45 (2006)

[55] R.B. Partridge and D.T. Wilkinson, Phys. Rev. Lett. 18, 557 (1967)

[56] J.M. Stewart and D.W. Sciama, Nature 216, 748 (1967).

[57] P.J.E. Peebles and D.T. Wilkinson, Phys. Rev. 174, 2168 (1968).

[58] A. Mészáros and Z. Molnár, Astrophys. J. 470, 49 (1996).

[59] M.J. Rees and D.W. Sciama, Nature 217, 511 (1968).

[60] S. Jha, A.G. Riess, and R.P. Kirshner, Astrophys. J. 659, 122 (2007).

[61] C. Gordon, K. Land, and A. Slosar, Mon. Not. R. Astr. Soc. 387, 371 (2008).

[62] P.J.E. Peebles, Principles of Physical Cosmology, (Princeton University Press, 1993).

[63] E.V. Linder, Phys. Rev. D 72, 043529 (2005).

[64] M. Bilicki, M. Chodorowski, G.A. Mamon, and T. Jarrett, Astrophys. J. 741, 31 (2011).

[65] B.M. Leith, S.C.C. Ng, and D.L. Wiltshire, Astrophys. J. 672, L91 (2008).

[66] P.R. Smale and D.L. Wiltshire, Mon. Not. R. Astr. Soc. 413, 367 (2011).

[67] P.R. Smale, Mon. Not. R. Astr. Soc. 418, 2779 (2011).

[68] J.A.G. Duley, M.A. Nazer, and D.L. Wiltshire, Class. Quantum Grav. 30, 175006 (2013).

[69] T. Buchert, Gen. Relativ. Grav. 32, 105 (2000).

[70] T. Buchert, Gen. Relativ. Grav. 40, 467 (2008).

[71] R. Keisler, C.L. Reichardt, K.A. Aird, et al., Astrophys. J. 743, 28 (2011).

[72] P.A.R. Ade, et al., arXiv:1303.5076 (2013).

[73] M. Maturi, K. Dolag, A. Waelkens, V. Springel, and T. Ensslin, Astron. Astrophys. 476, 83 (2007).

[74] K. Bolejko, Gen. Relativ. Grav. 41, 1737 (2009).

[75] H. Alnes and M. Amarzguioui, Phys. Rev. D 74, 103520 (2006).

[76] J. Grande and L. Perivolaropoulos, Phys. Rev. D 84 023514, (2011).

[77] K. Bolejko and J.S.B. Wyithe, J. Cosmol. Astropart. Phys. 02 (2009) 020.

[78] K. Bolejko, private communication.

[79] K. Bolejko, M.A. Nazer and D.L. Wiltshire, in preparation.

[80] P. Szekeres, Commun. Math. Phys. 41, 55 (1975).

[81] K. Bolejko and M.N. Célérier, Phys. Rev. D 82, 103510 (2010).

[82] K. Bolejko, Gen. Relativ. Grav. 41, 1585 (2009).

[83] R.A. Sussman and K. Bolejko, Class. Quantum Grav. 29, 065018 (2012)

[84] N. Kaiser, Mon. Not. R. Astr. Soc. 227, 1 (1987).

[85] J. Guy, P. Astier, S. Nobili, N. Regnault, and R. Pain, Astron. Astrophys. 443, 781 (2005).

[86] J. Guy, P. Astier, S. Baumont, et al., Astron. Astrophys. 466, 11 (2007).

[87] M. Kowalski, D. Rubin, G. Aldering, et al., Astrophys. J. 686, 749 (2008).

[88] M. Hicken, W.M. Wood-Vasey, S. Blondin, et al., Astrophys. J. 700, 1097 (2009).

[89] R. Amanullah, C. Lidman, D. Rubin, et al., Astrophys. J. 716, 712 (2010).

[90] R. Kessler, A. Becker, D. Cinabro, et al., Astrophys. J. Suppl. 185, 32 (2009).

[91] I. Zehavi, A.G. Riess, R.P. Kirshner, and A. Dekel, Astrophys. J. 503, 483 (1998).
[92] A. Conley, R.G. Carlberg, J. Guy, D.A. Howell, S. Jha, A.G. Riess, and M. Sullivan, Astrophys. J. 664, L13 (2007).

[93] I. Finkelman, N. Brosch, A.Y. Kniazev, et al., Mon. Not. R. Astr. Soc. 390, 969 (2008).

[94] I. Finkelman, N. Brosch, A.Y. Kniazev, et al., Mon. Not. R. Astr. Soc. 409, 727 (2011)

[95] A.G. Riess, L. Macri, S. Casertano, et al., Astrophys. J. 730, 119; (E) 732, 129 (2011).

[96] W. Percival, B.A. Reid, D.J. Eisenstein, et al., Mon. Not. R. Astr. Soc. 401, 2148 (2010).

[97] F. Beutler, C. Blake, M. Colless, et al., Mon. Not. R. Astr. Soc. 416, 3017 (2011).

[98] C. Blake, E. Kazin, F. Beutler, et al., Mon. Not. R. Astr. Soc. 418, 1707 (2011).

[99] H.K. Eriksen, F.K. Hansen, A.J. Banday, K.M. Gorski, and P.B. Lilje, Astrophys. J. 605, 14; (E) 609, 1198 (2004).

[100] A. de Oliveira-Costa, M. Tegmark, M. Zaldarriaga, and A. Hamilton, Phys. Rev. D 69, 063516 (2004).

[101] D.J. Schwarz, G.D. Starkman, D. Huterer, and C.J. Copi, Phys. Rev. Lett. 93, 221301 (2004).

[102] C.J. Copi, D. Huterer, D.J. Schwarz, and G.D. Starkman, Mon. Not. R. Astr. Soc. 367, 79 (2006).

[103] P.E. Freeman, C.R. Genovese, C.J. Miller, R.C. Nichol, and L. Wasserman, Astrophys. J. 638, 1 (2006).

[104] N. Hand, G.E. Addison, E. Aubourg, et al., Phys. Rev. Lett. 109, 041101 (2012).

[105] G. Lavaux, N. Afshordi, and M.J. Hudson, Mon. Not. R. Astr. Soc. 430 1617, (2013).

[106] R. Keisler, Astrophys. J. 707, L42 (2009).

[107] S.J. Osborne, D.S.Y. Mak, S.E. Church, and E. Pierpaoli, Astrophys. J. 737, 98 (2011).

[108] F. Atrio-Barandela, A. Kashlinsky, H. Ebeling, D. Kocevski, and A. Edge, Astrophys. J. 719, 77 (2010).

[109] F. Atrio-Barandela, A. Kashlinsky, H. Ebeling, and D. Kocevski, arXiv:1211.4345 (2012).

[110] P.A.R. Ade, et al., arXiv:1303.5090 (2013).

[111] F. Atrio-Barandela, Astron. Astrophys. 557, A116 (2013).

[112] G.F.R. Ellis and J.E. Baldwin, Mon. Not. R. Astr. Soc. 206, 377 (1984).

[113] P.A.R. Ade et al., arXiv:1303.5087 (2013).

[114] M. Rubart and D.J. Schwarz, Astron. Astrophys. 555, A117 (2013).

[115] C. Blake and J. Wall, Nature 416, 150 (2002).

[116] A.K. Singal, Astrophys. J. 742, L23 (2011).

[117] C. Gibelyou and D. Huterer, Mon. Not. R. Astr. Soc. 427, 1994 (2012).

[118] M.A. Abramowicz, S. Bajtlik, J.P. Lasota, and A. Moudens, Acta Astronomica 57, 139 (2007).

[119] M. Chodorowski, Mon. Not. R. Astr. Soc. 378, 239 (2007).

[120] Grøn, Ø. and Elgarøy, Ø., Am. J. Phys. 75, 151 (2007).

[121] M.J. Francis, L.A. Barnes, J.B. James, and G.F. Lewis, Publ. Astron. Soc. Austral. 24, 95 (2007).

[122] G.F. Lewis, M.J. Francis, L.A. Barnes, and J.B. James, Mon. Not. R. Astr. Soc. 381, L50 (2007).

[123] E.F. Bunn and D.W. Hogg, Am. J. Phys. 77, 688 (2009).

[124] B.F. Roukema, Mon. Not. R. Astr. Soc. 404, 318 (2010).

[125] V. Faraoni, Gen. Relativ. Grav. 42, 851 (2010).

[126] P.R. Smale, PhD thesis, (Univ. of Canterbury, 2012), http://hdl.handle.net/10092/6868. 